\title{
Preliminary Design of an Intermittent Smoke Flow Visualization System
}

Donald T. Ward and James H. Myatt

GRANT NAG-2651

June 1993

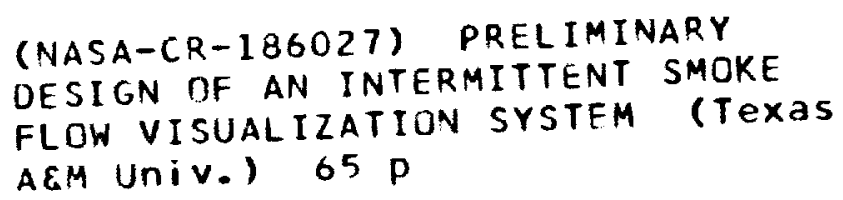

N93-28693

Unclas

G3/05

0171942

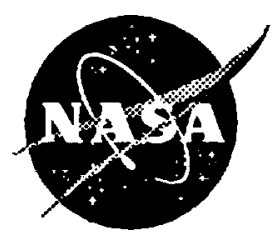

National Aeronautics and Space Administration 
NASA Contractor Report 186027

\section{Preliminary Design of an Intermittent Smoke Flow Visualization System}

Donald T. Ward and James H. Myatt

Texas A \& M University

College Station, Texas

Prepared for

Dryden Flight Research Facility under Grant NAG-2651

1993

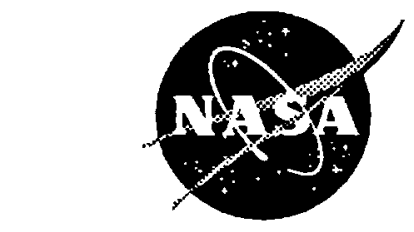

National Aeronautics and

Space Administration

Dryden Flight Research Facllity

Edwards, California 93523-0273 
、 


\section{TABLE OF CONTENTS}

Title of Section

Rage Number

List of Tables..

.iii

List of Figures............................................................................................

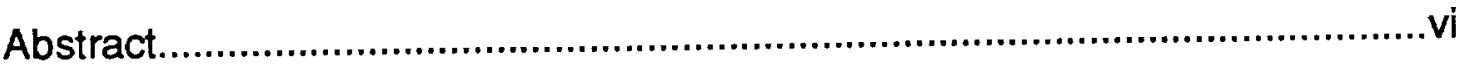

List of Symbols and Acronyms....................................................................ii

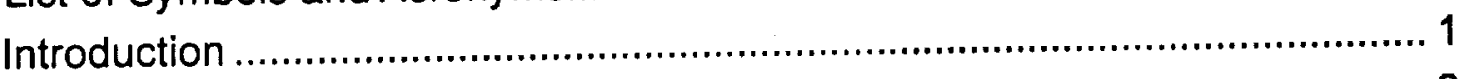

Essential Elements for the ISFVS..................................................... 2

Seeding Medium ............................................................................ 2

Data Collection ........................................................................... 2

Preliminary Design and Development....................................................... 4

System Requirements..................................................................... 4

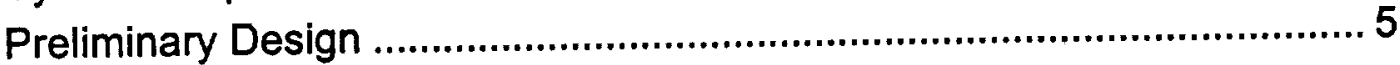

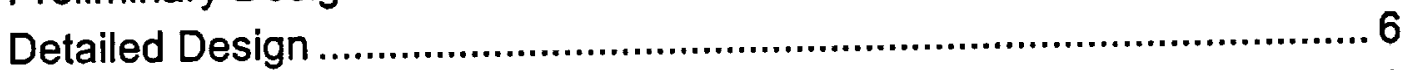

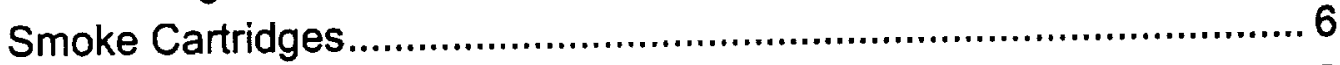

Smoke Cartridge Container and Plenum Chamber .............................. 6

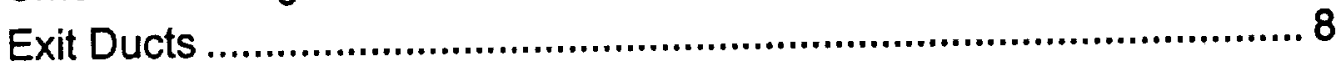

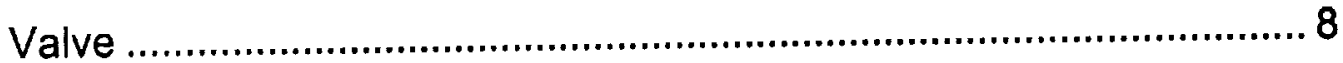

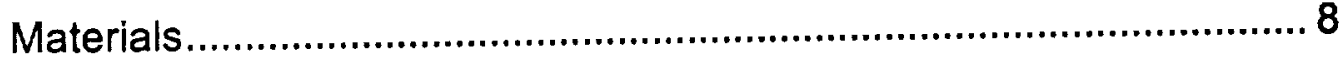

Determination of Wall Thicknesses................................................ 9

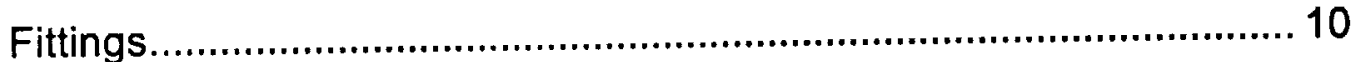

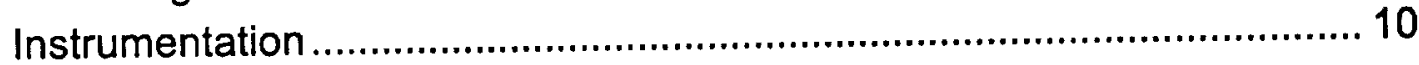

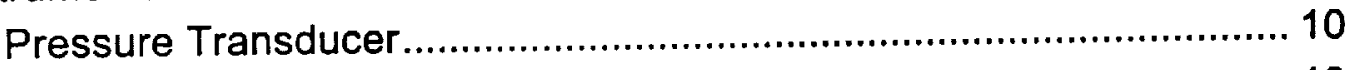

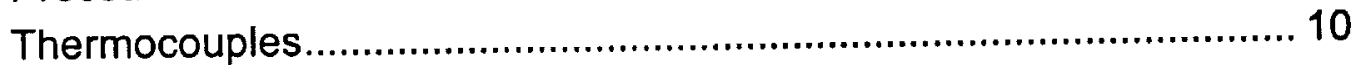

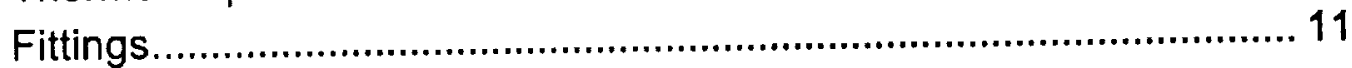

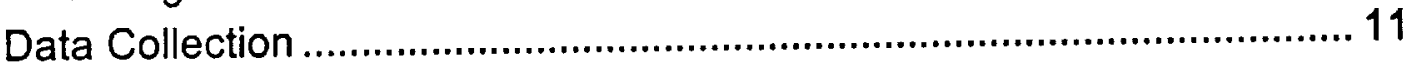

Signal Conditioning ......................................................................... 11

Synchronizing Cartridge and Data Collection .................................... 11

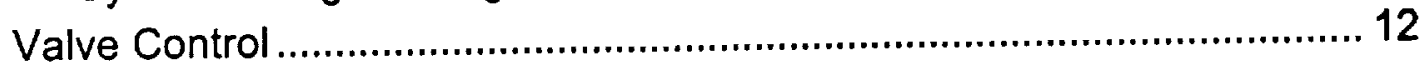

Pressure Estimation .......................................................................... 12

Chemical Reaction........................................................................... 12

Adiabatic Flame Temperature of Reaction ............................................. 14

Thermodynamic Properties of Terephthalic Acid.................................... 16

Mixture Properties............................................................................. 17

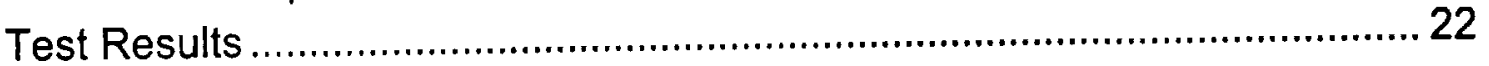


Title of Section

Experimental Procedure .................................................................. 22

Experimental Results........................................................................... 23

Pressure Measurements at the Transducer Stand-off.......................... 23

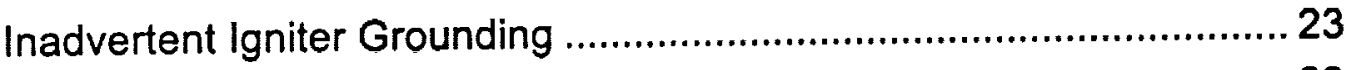

Valve Reliability............................................................................... 23

Coherence and Discreteness of Smoke Puffs................................... 24

System Temperatures ..................................................................... 26

System Pressure ............................................................................. 27

Comparison of Predicted and Measured Pressures and Velocities............ 31

Effect of Smoke on the Flow Field ........................................................... 35

Conclusions and Recommendations..........................................................36

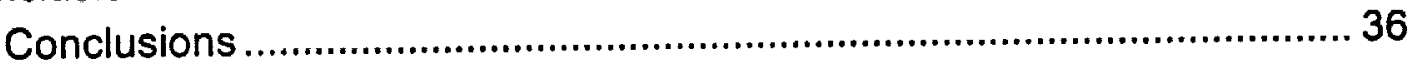

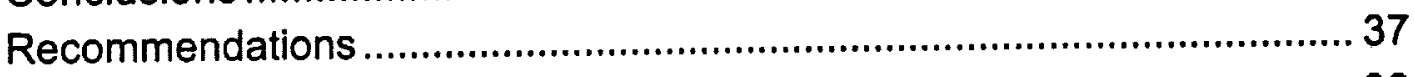

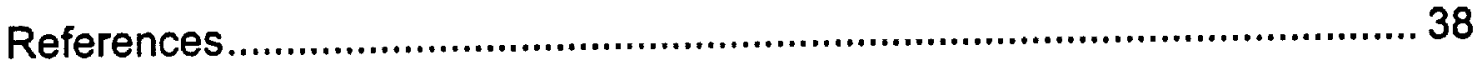

Appendix A FORTRAN Code for Modeling System Pressure and Exit

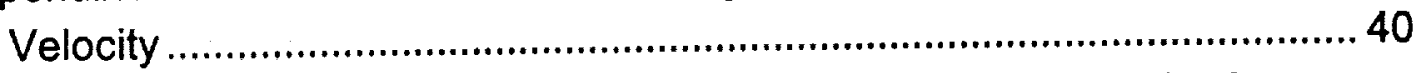

Appendix B Detailed Drawings of Intermittent Smoke Flow Visualization

Generator Prototype 


\section{LIST OF TABLES}

Table Number Title

Page Number

1 Common Seeding Media........................................................................ 3

2 Tubing Thicknesses............................................................................ 10

3 Smoke Cartridge Reactants ................................................................. 13

4 Products of Sucrose- $\mathrm{KClO}_{3}$ Reaction and Decomposition of

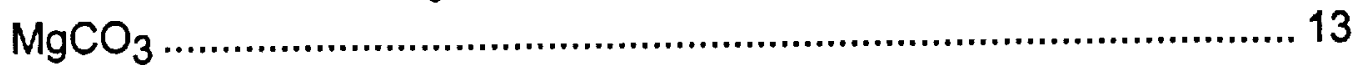

5 Products of Smoke Cartridge Reaction ...................................................... 14

6 Physical Properties of Smoke Cartridge Products ...................................... 14

7 Heat of Formation of the Reactants ....................................................... 15

8 Reaction Products' Heat of Formation and Change in

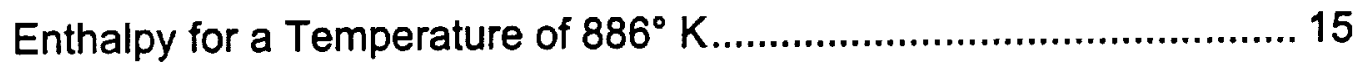

9 Mixture Composition .......................................................................... 18

10 Mass Fractions and Mole Fractions for the Smoke Mixture

Compounds ............................................................................... 19

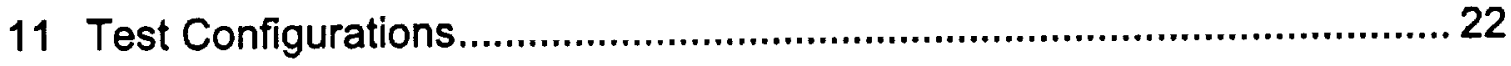

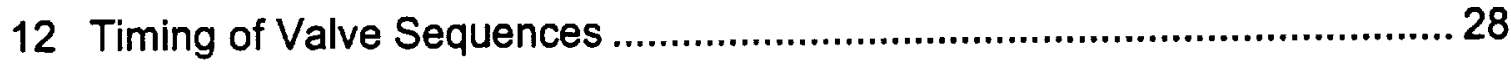

13 Maximum Undisturbed Plenum Chamber Pressure

Differential

14 System Parameters for Comparison of Pressures and

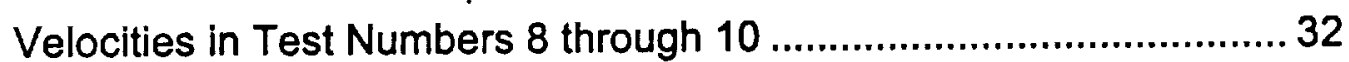

15 Smoke Generator Parts List............................................................... 53 


\section{LIST OF FIGURES}

Figure Number Figure Title

Page Number

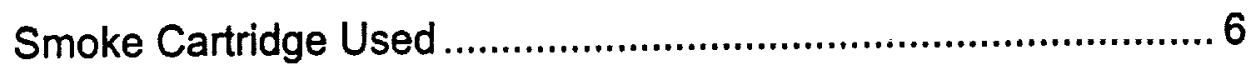

Smoke Cartridge Container and Cap ............................................ 7

Container Cap and Plenum Inlet Plate Subassembly 7

Plenum Chamber Subassembly 8

Three-Way Double Solenoid Switching Valve

Stresses in a Cylinder Wall. 9

Ideal Gas Heat Capacity of Terephthalic Acid 16

Ideal Gas Total Enthalpy of Terephthalic Acid

Enthalpy of Terephthalic Acid for Use in Conservation of Energy

Specific Enthalpy of Terephthalic Acid Used in Conservation

of Energy

Internal Energy of the Mixture...

Thermodynamic Control Volume 20

Gas Flow Graph for Low Pressure Drop Across Valve 21

Gas Flow Graph for High Pressure Drop Across Valve 22

Temperature at Pressure Transducer Stand-off.

Camera 1 View of a Typical Smoke Puff Leading Edge. 24

Camera 2 View of a Typical Smoke Puff Leading Edge 25

Downstream Location of Smoke Puff Leading Edge 26

Temperature of Gaseous Mixture Exiting the Smoke Cartridge ... 26

Temperature of Gaseous Mixture in the Plenum Chamber .......... 26

Temperature of Smoke at the Duct Exit

Plenum Chamber Pressure Differential with Smoke Flow

Diverted

Plenum Chamber Pressure During Test Number 8

Plenum Chamber Pressure During Test Number 9

Plenum Chamber Pressure Differential During Six Cycles of

Test Number 9

Measured Smoke Exit Velocity During Test Number 9

Measured Smoke Exit Velocity During Test Number 10 


\section{LIST OF FIGURES (Continued)}

Figure Number Figure Title

Page Number

32

33

34

35

36

37

38

39

40

41

42

43

44

45

46

47

48

49

50

51

52

53

54

Comparison of Measured and Predicted Plenum Chamber

Pressure During Test Number 8

Comparison of Measured and Predicted Plenum Chamber

Pressure During Test Number 8

Comparison of Measured and Predicted Plenum Chamber

Pressure During Test Number 9

Comparison of Measured and Predicted Plenum Chamber

Pressure During Test Number 9

Measured and Predicted Smoke Exit Velocities (Test 9) ............. 32

Measured and Predicted Smoke Exit Velocities (Test 9) .............. 33

Measured and Predicted Plenum Chamber Pressures

(Test 10)

Measured and Predicted Plenum Chamber Pressures

(Test 10)

Measured and Predicted Smoke Exit Velocities (Test 10) ........... 34

Measured and Predicted Smoke Exit Velocities (Test 10) ............ 34

Mass Flow Rate of Smoke through the Open Valve..................... 34

Blowing Momentum Coefficient of Smoke at $q_{\infty}=18.28 \mathrm{psf}$

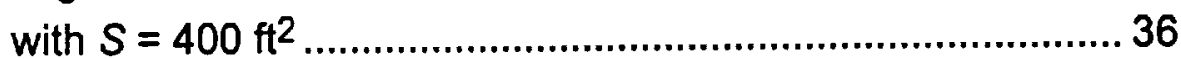

Exploded Isometric View of the Prototype ISFVS ....................... 41

Detailed Drawing of the Bottom Ring Clamp.............................. 42

Detailed Drawing of the Bottom of the Cartridge Container ........ 43

Detailed Drawing of the Cartridge Container and Retainer......... 44

Detailed Drawing of the Top of the Cartridge Container ............. 45

Detailed Drawing of the Cartridge Cap ..................................... 46

Detailed Drawing of the Tube Connecting the Container Cap

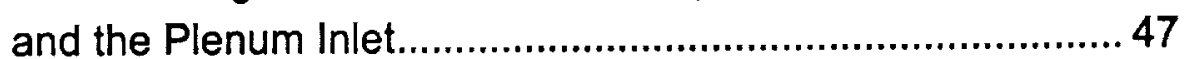

Detailed Drawing of the Plenum Chamber Inlet Plate ................. 48

Detailed Drawing of the Plenum Chamber .................................. 49

Detailed Drawing of the Plenum Chamber Exit Plate ................... 50

Detailed Drawing of the of the AN Fitting Assembly on the

Plenum Chamber Exit Plate. 


\title{
PRELIMINARY DESIGN OF AN INTERMITTENT SMOKE FLOW VISUALIZATION SYSTEM
}

\begin{abstract}
A prototype intermittent flow visualization system has been designed to study vortex flow field dynamics has been constructed and tested through its ground test phase. It produces discrete pulses of dense white smoke consisting of particles of terephthalic acid by the pulsing action of a fast-acting three-way valve. The trajectories of the smoke pulses can be tracked by a video imaging system without intruding in the flow around in flight. Two methods of pulsing the smoke were examined. The simplest and safest approach is to simply divert the smoke between the two outlet ports on the valve; this approach should be particularly effective if it were desired to inject smoke at two different locations during the same test event. The second approach involves closing off one of the outlet ports to momentarily block the flow. This second approach requires careful control of valve dwell times to avoid excessive pressure buildup within the cartridge container and does also increase the velocity of the smoke injected into the flow. The flow of the smoke has been blocked for periods ranging from 30 to 80 milliseconds, depending on the system volume and the length of time the valve is allowed to remain open between valve closings. The maximum differential pressure as a result of such intermittent blockage of the smoke flow is 17 psid. For this pressure differential the blowing momentum coefficient for an aircraft with a wing reference area of 400 square feet flying at 30,000 feet and 120 knots is approximately 0.0017 , well below the experimental value for blowing momentum coefficient that has caused major disruptions in vortical flow patterns over strakes or leading edge extensions. However, the acceptable maximum blowing momentum coefficient to avoid disturbing the forebody vortical flow field is uncertain and deserves additional study. A mathematical simulation of the pressures and velocities accurately predicted these parameters so long as suitable initial conditions were known. Ten cartridges were fired, all with no external flow other than a small fan to clear the expelled gases, to prove the concept in initial ground tests. Video data of these static firings indicate that the smoke puffs can be readily tracked by high speed video cameras. It is strongly recommended that this prototype design be further refined and validated in the wind tunnel and in flight.
\end{abstract}




\section{LIST OF SYMBOLS AND ACRONYMS}

\begin{tabular}{|c|c|}
\hline Symbol & Dimensions \\
\hline$A$ & Area \\
\hline AOA or $\alpha$ & Angle of Attack \\
\hline$c_{P}{ }^{0}$ & Heat Capacity ..............................................................none \\
\hline$c_{\mu}$ & Blowing Momentum Coefficient ......................................none \\
\hline$E^{\mu}$ & Total System Energy \\
\hline$F$ & Flow Rate Correction Factors ........................................none \\
\hline$H$ & 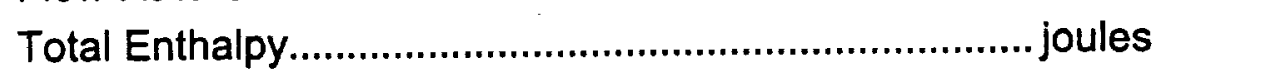 \\
\hline$M$ & Molecular Weight................................................atomic mass units \\
\hline$N$ & Number of Moles (or Gram-Moles) ...................................none \\
\hline$P$ & 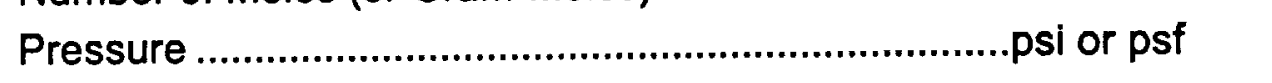 \\
\hline$Q$ & Heat Added to a Thermodynamic System ....................... joules \\
\hline$R$ & 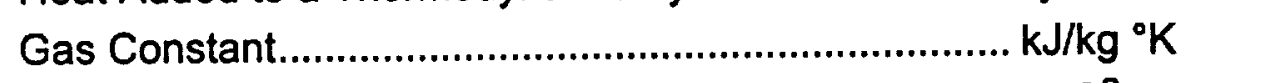 \\
\hline$s$ & Wing Planform Area ........................................................... $\mathrm{ft}^{2}$ \\
\hline SFCH & Standard Flow Rate Through Valve ............................. $\mathrm{ft}^{3} / \mathrm{hr}$ \\
\hline$T$ & Temperature \\
\hline$V$ & 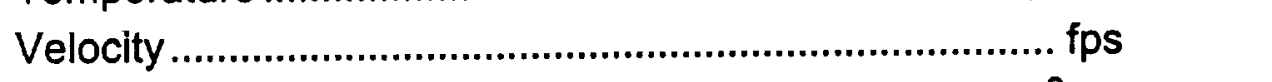 \\
\hline$V$ & 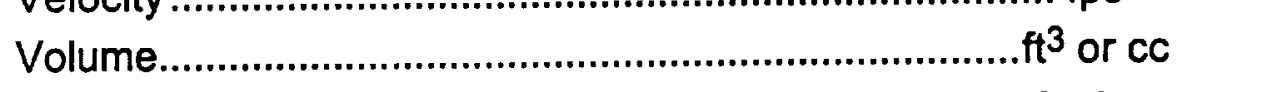 \\
\hline$W$ & Work Done by a Thermodynamic System .......................joules \\
\hline$\theta$ & Specific Energy........................................................joules/gram \\
\hline$h$ & Specific Enthalpy .......................................................... \\
\hline$m$ & 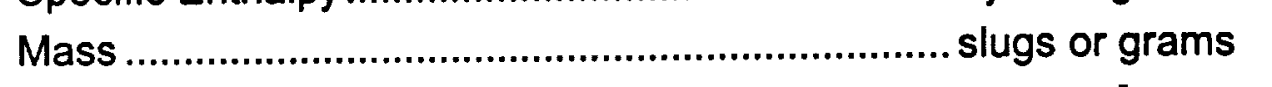 \\
\hline$q$ & Dynamic Pressure ............................................................. psf \\
\hline$r$ & Radius \\
\hline$t$ & 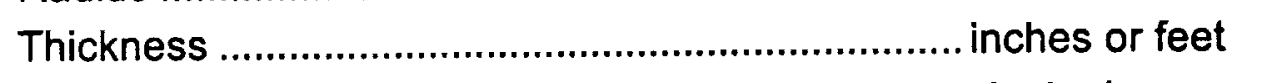 \\
\hline$u$ & Specific Internal Energy...........................................joules/gram \\
\hline$x$ & Cylindrical Coordinate Direction ......................................none \\
\hline$y$ & 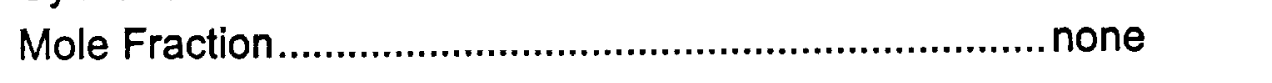 \\
\hline $\mathbf{z}$ & Cylindrical Coordinate Direction .....................................none \\
\hline$\theta$ & Cylindrical Coordinate Direction ........................................none \\
\hline$\rho$ & 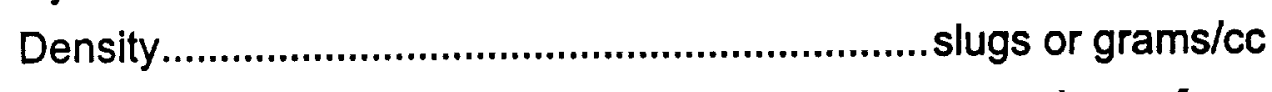 \\
\hline$\sigma$ & Stress \\
\hline
\end{tabular}




\section{LIST OF SYMBOLS AND ACRONYMS (Continued)}

\begin{tabular}{cl} 
Subscript & Description \\
\hline$G$ & Gas Flow Graph Reading \\
$T$ & Products \\
$R$ & Reactants \\
$S G$ & Specific Gravity Correction \\
$T$ & Temperature Correction Factor \\
actual & Actual \\
$\theta$ & Exit Conditions \\
$f$ & Formation \\
$i$ & Inside Dimension or ith Component or Inlet Condition \\
$k$ & Kinetic Energy Index \\
$m$ & Summation Index or Mixture \\
max & Maximum \\
min & Minimum \\
$n$ & Normal Direction \\
0 & Outside Dimension \\
$p$ & Potential Energy Index \\
ref & Reference Condition \\
$s$ & Shaft \\
$\cdot$ & Time Derivative \\
$1,2, \ldots$ & Indices \\
$\theta$ & Tangential Direction \\
$\infty$ & Freestream Conditions
\end{tabular}


LIST OF SYMBOLS AND ACRONYMS (Continued)

Acronym Description

A-DFRF Ames-Dryden Flight Research Facility

AVD Analog-to-Digital

D/A Digital-to-Analog

DMA Direct Memory Access

FML Flight Mechanics Laboratory

HARV High Angle of Attack Research Vehicle

I.D. Inside Diameter

ISFVS Intermittent Smoke Flow Visualization System

NASA National Aeronautics and Space Administration

NPT National Pipe Thread

SAE Society of Automotive Engineers

SGS Smoke Generator System

TIG Tungsten Inert Gas 


\section{PRELIMINARY DESIGN OF AN INTERMITTENT SMOKE FLOW VISUALIZATION SYSTEM}

\section{INTRODUCTION}

Aircraft designers solving problems associated with high angle of attack (AOAs) maneuvering rely heavily on data obtained in flight. The High AOA Research Program at the NASA Ames-Dryden Flight Research Facility (A-DFRF) is currently investigating the high AOA characteristics of a modified F/A-18 and the X-29. The purpose of this ambitious program is to provide high AOA flight data, confirm theoretical methods, identify and solve control problems, and validate agility measures of merit. These experiments will help aircraft designers build aircraft which are highly maneuverable, even in flight regimes where current fighters cannot safely operate ${ }^{1}$.

A major part of this effort is focused on the vortical flow developed on the forebody and leading edge extensions of the F/A-18. A smoke generating system has been installed on the F/A-18 test vehicle or the High Alpha Research Vehicle (HARV). This smoke system releases a continuous stream of dense white smoke through ports in the skin of the aircraft into the airflow upstream of the area of interest. The smoke then follows the airflow as it travels aft over the aircraft, allowing visualization of the vortices. The dynamic positions of the vortices and the burst point locations can be accurately determined by analyzing video tape and motion picture film of the smoke.

The HARV Smoke Generator System (SGS) uses chemical cartridges which were designed and manufactured by the U.S. Army Chemical Research, Development, and Engineering Center. They consist of metal containers filled with a pyrotechnic chemical mixture which is ignited with electric matches ${ }^{2}$. The chemical mixture contains terephthalic acid (also known as 1,4-benzenedicarboxylic acid), sugar, potassium chlorate, and other ingredients in small amounts to improve handling qualities and burn rate. The sugar (fuel) and potassium chlorate (oxidizer) react to produce heat. The heat causes the terephthalic acid to vaporize. The vapor causes a pressure increase inside the cartridge, forcing the vapor out of the cartridge. Once away from the heat of the reaction the gas cools until it condenses ${ }^{10}$ at a temperature of $572^{\circ} F^{4,5}$ to form the solid particles which make up the visible smoke.

Six cartridges are mounted in a housing and placed in the gun bay of the F/A-18 HARV. The gun bay was chosen because of its location near the origin of the vortex cores, and it is also designed to withstand high temperatures. The smoke from each of the six cartridges is ducted to a common plenum chamber. The smoke is then ducted to a port in the aircraft skin where it is released into the air flow upstream of the area of interest. All tubing from the SGS to the exterior ports is 0.93 inches inside diameter.

The SGS has been successfully tested in flight at altitudes ranging from 18,000 to 33,000 feet, indicated airspeeds of 100 to 140 knots, and while performing several maneuvers, including steady state flight at an angle of attack of $20^{\circ}$ and in angle of attack sweeps between $15^{\circ}$ and $35^{\circ}$. Firing two cartridges simultaneously produced smoke of sufficient density for approximately thirty seconds. The smoke trajectory was recorded 
using video cameras and still cameras mounted on the test aircraft, and it provided ample contrast for identifying the vortex core. However, it was not possible to obtain video data from any video camera for which the sun was in the field of view, which can occur during wind-up turns or other maneuvers where a constant heading is not maintained. Multiple cameras mounted in different locations should reduce the amount of lost data. The automated data reduction hardware/software system used at Texas A\&M University ${ }^{6}$ permits analyses using up to three different correlated video input channels. Provision for a fourth video channel is included in the system, but the hardware to support this fourth has not been purchased.

Because the current NASA smoke generator system releases a continuous stream of smoke, the obtainable information is limited to the positions of streamlines and vortex cores, as well as burst point locations. However, if the smoke were to be released in discrete pulses, and if the fluid packets remained coherent, the path of each pulse can be tracked as it flows over the aircraft. Once the position of a fluid element is found as a function of time, the velocity and acceleration of the pulse of smoke as it moves over the aircraft can be found by numerical differentiation. Such information has been very useful in studying the effects of the vortex core in water tunnels and in wind tunnels 7,8 .

The central requirement for a smoke generating system which can be used to obtain such fluid dynamic data is that it must produce discrete elements of the seeding material which remain discrete as they travel through the flow field. Although it is desirable to have smoke elements which are short enough in length that the entire element can be tracked, it is not necessary. Alternatively, the leading edge or trailing edge of an element can be tracked, and this procedure may even be preferable in some cases.

This report details the preliminary development of such an intermittent flow visualization system through its design and initial ground tests. The report discusses selection of a visualization medium, design and construction of the hardware necessary for a ground test, instrumentation of the system to determine its safety, and static ground tests of the system to ensure that it produces discrete elements of the visualization medium which will not significantly disturb the flow field. This phase of the effort includes no wind tunnel tests nor flight tests that will verify the feasibility of using the video imaging system to collect the dynamic velocity and acceleration data described above; those verifications of the concept are planned for Phase 2 of the project.

\section{Essential Elements for the ISFVS}

Seeding Medium. One of the critical elements of any flow visualization system is the seeding medium. Commonly used materials used for seeding air flow and their advantages and disadvantages for use in visualizing vortical flows in flight are listed in Table I. Obviously, there is no perfect medium; all candidates have disadvantages.

Data Collection. Another important factor in producing a workable ISFVS is the data collection scheme. The development of video imaging systems, which can be used to track objects in a three-dimensional space, has largely automated the quantification of flow parameters. 
Table 1. Common Seeding Media

\begin{tabular}{|c|c|c|}
\hline $\begin{array}{l}\text { Method producing } \\
\text { seeding medium }\end{array}$ & Advantages & Disadvantages \\
\hline $\begin{array}{l}\text { heating mineral oils to } \\
\text { vaporization } 9,10\end{array}$ & non-toxic and non-corrosive & $\begin{array}{l}\text { may not produce enough smoke to fill } \\
\text { the vortex core; } \\
\text { requires heat produced by the } \\
\text { airplane engine or electrical system }\end{array}$ \\
\hline $\begin{array}{l}\text { bringing titanium tetrachlo- } \\
\text { ride into the presence of } \\
\text { moist air } 9,10\end{array}$ & $\begin{array}{l}\text { gives clearly visible smoke } \\
\text { with high contrast levels }\end{array}$ & $\begin{array}{l}\text { produces highly corrosive fumes; } \\
\text { suitable only for air speeds on the } \\
\text { order of } 5 \text { feet per second }\end{array}$ \\
\hline soap bubbles 9,10 & non-toxic and non-corrosive & $\begin{array}{l}\text { maximum speed for using soap } \\
\text { bubbles is about } 60 \text { miles per hour; } \\
\text { not likely to produce discernible } \\
\text { contrast levels }\end{array}$ \\
\hline $\begin{array}{l}\text { burning wood, tobacco, or } \\
\text { paper }^{9,10}\end{array}$ & $\begin{array}{l}\text { fuel is cheap and readily } \\
\text { available }\end{array}$ & open flame is not acceptable in flight \\
\hline $\begin{array}{l}\text { mixing anhydrous ammonia } \\
\text { and sulfur dioxide }{ }^{11}\end{array}$ & uses common chemicals & $\begin{array}{l}\text { may not produce enough smoke to fill } \\
\text { the vortex core }\end{array}$ \\
\hline chemical cartridges 2,12 & $\begin{array}{l}\text { some types produce very } \\
\text { dense smoke; } \\
\text { different colors of smoke } \\
\text { available; } \\
\text { chemical mixture can be } \\
\text { tailored to provide desired } \\
\text { results }\end{array}$ & $\begin{array}{l}\text { high temperatures may be associated } \\
\text { with the chemical reaction; } \\
\text { once fired, a cartridge cannot be shut } \\
\text { down - it must bum out; } \\
\text { may produce unpleasant odor or } \\
\text { deposit residue that is unacceptable }\end{array}$ \\
\hline
\end{tabular}

The video imaging system used by the Texas A\&M University Flight Mechanics Laboratory (FML) consists of hardware (a video processor, cameras, video records, etc.) and a versatile software package. Video images from either the cameras or from video tapes are sent to the video processor, which measures the intensity of each pixel in each frame and compares it to the intensities of adjacent pixels. If the difference in intensity between neighboring pixels is above a threshold level set by the operator, the pixel location is identified as an area of contrast. The pixel locations for areas of contrast are recorded for each frame. When a light-colored object passes by a dark background (or vice versa), the edges of the object are thus "seen" by the video processor as lines of contrast. This video processor can also be configured to only identify a top, bottom, left, or right edge (or any combination of these edges). The software package is then used to find any area within a frame which has a minimum number of contrasting pixels in it. The centroids of these areas are recorded for each frame. The dimensions of the area and the minimum or maximum number of pixels to be considered as a distinct object are also set by the user from a convenient menu. Once the centroids are found, the trajectory of the object(s) is determined by comparing similar objects in succeeding frames and linking them together in a path. Since the frame rate is known accurately, the time history of the trajectory is defined. The velocity and acceleration of the object(s) can then be found using digital smoothing filters and numerical differentiation commands that are integral to the software ${ }^{6}$.

The only equipment that must be carried on the test aircraft are video cameras that can record the flow visualization medium in the area of interest. For best results these 
camera lines of sight should be parallel as nearly as possible to three orthogonal coordinate directions. While two cameras can produce three-dimensional data, it is highly desirable to have multiple camera locations to provide statistical redundancy in the lines of position that determine target positions. Since the tracking algorithms are based on detection of contrast levels to define the targets, lighting and background of the video scene is also critically affect the quality of the data. The system can track over 30 different target locations simultaneously in any one area of interest, though obviously the number of targets to be tracked simultaneously directly affects the size of the data files to be reduced.

\section{PRELIMINARY DESIGN AND DEVELOPMENT}

In fulfillment of the statement of work for NAG 2-651 the fabrication and ground tests of a smoke generating system which can be used to introduce discrete elements of a flow visualization medium into the flow field of an aircraft in flight have been completed. As dictated in the statement of work, the results presented in this report include: drawings and instructions for the assembly of a prototype intermittent smoke flow visualization system

discussion of the results of the tests showing that the system produces discrete elements of the visualization medium

engineering predictions of the pressure and temperatures produced in the system

experimental verification that the pressures and temperatures generated in the system can be easily and safely contained.

\section{System Requirements}

A system which can produce discrete elements of a seeding material for identifying the trajectories of vortex cores in flight must meet stringent requirements. These requirements fall into two categories, those concerned with the system's ability to produce desired results, and those concerned with its use on an aircraft in flight.

System performance requirements include:

- The system must produce elements of the flow visualization medium which are discrete and remain distinguishable from one another as they travel through the flow field.

- The seeding material should be introduced into the flow field so that it does not significantly disturb the experimental conditions ${ }^{13}$.

a The seeding material must be dense and contrast with the background so that it can be identified by the chosen means of data collection.

- Individual particles making up the visualization medium must be small enough and contain an appropriate amount of mass so that they follow the actual flow of the fluid around an aircraft flying at speeds near 120 knots indicated airspeed 10 . 
To be useful for testing during maneuvers, the system must produce usable smoke for at least twenty to thirty seconds ${ }^{2}$.

A flow visualization system for use on an aircraft must also meet other requirements:

The seeding material must be non-toxic and non-corrosive?.

The system may not produce unsafe pressures or temperatures.

Because of the high cost of conducting flight tests, the system must also be reliable.

The system must be compact and lightweight.

The system must be powered by and controlled from the aircraft.

\section{Preliminary Design}

Several different methods of producing discrete elements of a visualization medium were considered. A modification of NASA's HARV Smoke Generator System (SGS) was identified as the most promising. This approach was chosen because the HARV SGS worked well in flight during the initial phase of the High Alpha Research Program. The chemical cartridge introduces a non-toxic and non-corrosive smoke (from the condensation of terephthalic acid) into the flow field in quantities large enough to define a vortex core and provide good contrast with the HARV and the sky. Moreover, the original design has already been flight qualified, and it is safe and reliable. The data obtained using the HARV SGS compared well with computational fluid dynamics results; so, evidently the SGS did not significantly alter flow field characteristics ${ }^{14}$.

The most obvious way to introduce smoke into the flow field in discrete elements is to use a valve to shut off the flow of smoke for short periods of time. However, shutting off the flow of smoke causes pressure rises in the system. If the flow of smoke is blocked for too long, these pressure rises may cause damage to the system, or they may cause the smoke to enter the flow field with a velocity sufficient to disturb the vortical flow conditions. A measure of the effect of interrupting the flow of smoke on the exit velocity is the increase in the pressure differential between the inside and outside of the plenum. Increasing the internal volume of the system decreases the pressure rise which occurs as a result of shutting off the flow for a short time period. However, the volume which may be needed to ensure that the pressure rise is not too great may be too large to meet space requirements. Another way of introducing discrete elements of smoke into the flow field is to divert the smoke to an alternate outlet when it is not being sent to the region of the flow field being studied. The alternate outlet flow path may lead to another flow region which is being studied or any region of the flow where it will not interfere with the flow in the region of concern. The latter option will waste visualization material, but it may be necessary to preserve the integrity of the flow field.

Since the effect of blocking the smoke flow path, especially on pressure and temperature, was largely unknown at the beginning of the project, a three-way valve was sought. This type of valve would allow both diversion of the smoke to an alternate path, and complete blockage of the flow of smoke. The pressure rises caused by blocking 
the flow path must be analyzed and verified to determine which method of pulsing the smoke is best for a given application.

Because of NASA's success with the HARV SGS in the initial phase of the High Alpha Research Program, their existing design was used as the basis for the prototype system that would pulse the smoke. However, the prototype was kept simple by designing for only one smoke cartridge and the uncertainty surrounding the thermodynamic effects of opening and closing the valve led to designing a plenum chamber in
which the volume could be easily changed. This volume flexibility deliberately designed flexibility into the prototype to help meet what was perceived as the difficult design challenge.

\section{Detalled Design}

As suggested above, a single cartridge prototype with three different plenum chamber volumes was designed for use in Phase 1 ground tests. The NASA SGS design was otherwise duplicated as nearly as possible. The major components of the prototype design are discussed in detail below.

Smoke Cartridges. Smoke cartridges (Figure 1) were obtained from NASA A-DFRF. A steel canister similar to a U.S. Army hand smoke grenade holds 330 grams of a pyrotechnic chemical mixture. After the cartridge has been fired, less than $\mathbf{5 0}$ grams of residue remain ${ }^{2}$. The chemical mixture contains terephthalic acid (also known as 1,4benzenedicarboxylic acid), sugar, potassium chlorate, and other ingredients in small amounts to improve handling qualities and burn rate. The sugar (fuel) and potassium chlorate (oxidizer) react to produce heat. The heat causes the terephthalic acid to vaporize. The vapor causes a pressure increase inside the cartridge, forcing the vapor out of the cartridge. Once away from the heat of the reaction the gas cools until it condenses at a

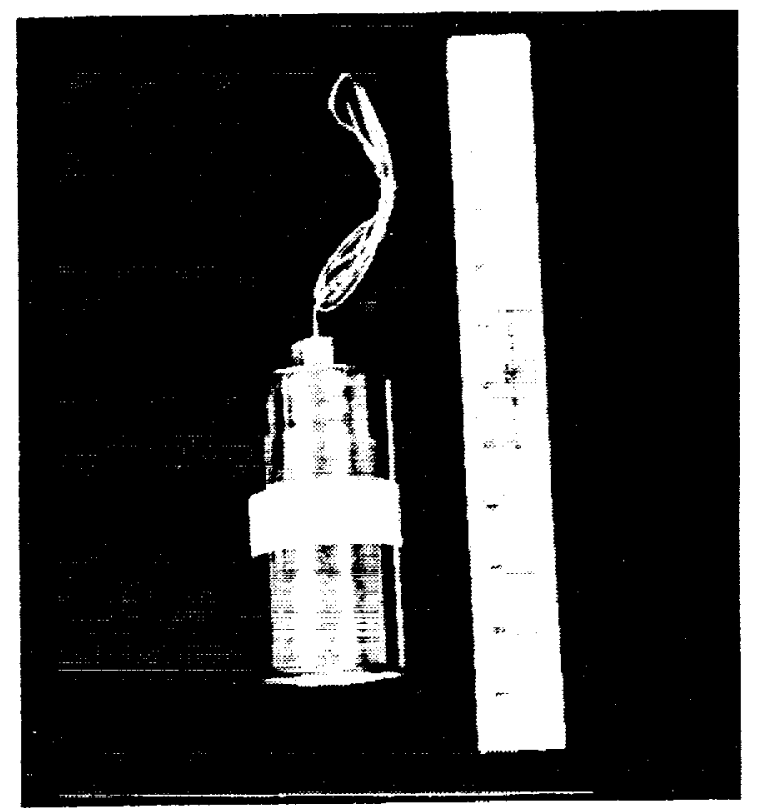

Figure 1. Smoke Cartridge Used temperature of $572^{\circ} \mathrm{F}^{10}$ to form the solid particles which make up the visible smoke 4,5 . The cartridges are ignited with an electric igniter which is screwed onto the top of the cartridge. A 28 volt, 1.3 amp current is needed for ignition. The smoke cartridges are 4.7 inches long and 2.4 inches in diameter. Four 0.31 inch diameter holes in the top allow the smoke to exit the cartridge. Before firing, the four holes are covered with aluminum tape which is forced off by the buildup of pressure when the cartridge is ignited.

Smoke Cartridge Container and Plenum Chamber. The smoke cartridge is placed in the cartridge retainer, which is mounted inside the cartridge container using stand- 
offs (Figure 2). Smoke exits the cartridge container through a 0.93 inch inside diameter tube in the container cap. The cap is nozzled to reduce pressure losses. The igniter wires run through a fitting in the container cap. An Igniter stand-off holds the cartridge firmly in place.

A rupture disk is placed in the bottom of the cartridge container. This rupture disk rapidly releases the smoke in the event of an excessive pressure buildup in the cartridge container. The rupture disk is designed to rupture at pressures between 55 and 75 psid $^{2}$.

A ring clamp is used to hold the rupture disk and rup-

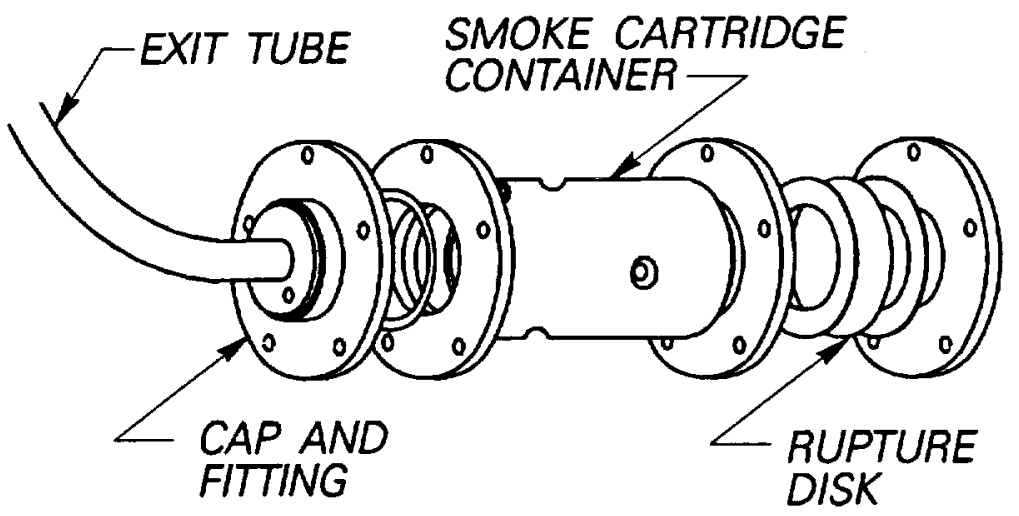

Figure 2. Smoke Cartridge Container and Cap ture disk gaskets in place. One ring is welded to the bottom of the cartridge container; the other ring is bolted to it using five $3 / 8^{\prime \prime} \times 1-1 / 4$ " SAE Grade 9 , fine thread bolts, nuts, and washers. The rupture disk and two gaskets are sandwiched between the two rings.

A ring clamp connects the cartridge container to its cap. One ring is welded to the container; the other is welded to the cap. The two rings are bolted together with five bolts. An O-ring is placed between the two rings to prevent leaks.

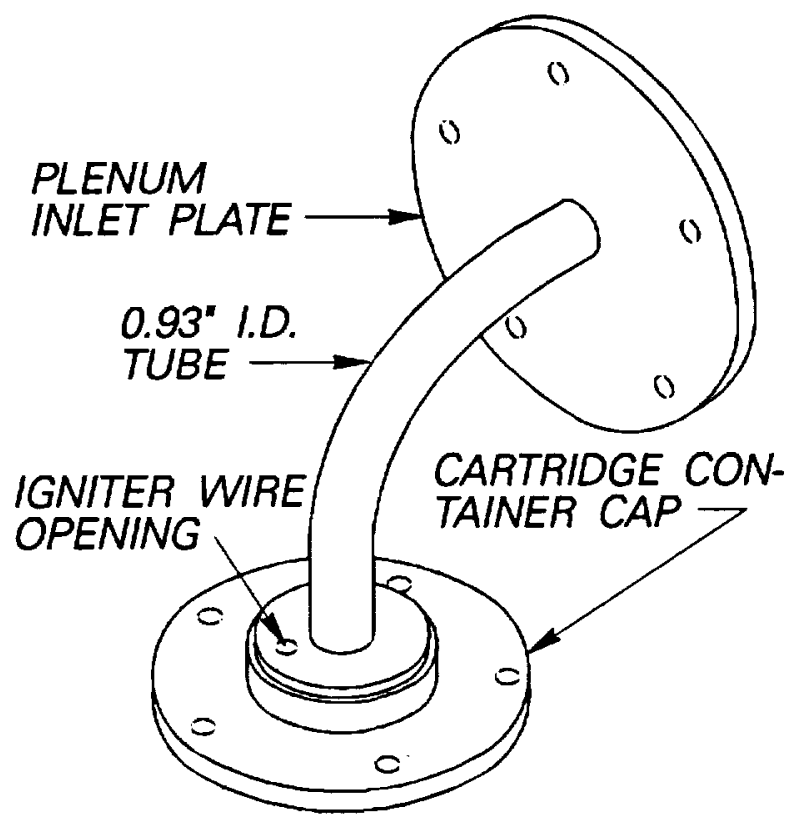

Figure 3. Container Cap and Plenum Inlet Plate Subassembly

A 0.93 inch inside diameter tube with a $4.0^{\prime \prime}$ radius, $90^{\circ}$ bend connects the container cap with the inlet plate of the plenum chamber. The tube is welded to both the container cap and the plenum inlet plate. Figure 3 shows how the exit tube (Figure 2) connects the smoke cartridge container to the plenum chamber inlet plate. All welds are tungsten inert gas (TIG) welds; the exterior welds were sealed with a high temperature sealant to prevent leaks. Also, the opening for the igniter wires (labeled in Figure 3 ) is sealed similarly.

The plenum chamber itself (Figure 4) consists of a cylinder and two end plates. The cylinder making up the walls of the plenum chamber is made of aluminum tubing and different lengths of tubing were cut to provide a simple and fast way to change the volume of the plenum chamber, an important parameter for controlling the pressure rise in the system as the fast-acting valve opens and closes to produce the intermittent smoke puffs. The exit plate of the plenum chamber is also nozzled to 
reduce pressure losses. The two end plates are held in place by five $3 / 8^{\prime \prime}$ SAE Grade 9 , fine thread bolts, nuts, and washers. Two O-rings ensure that there is no leakage between the tube and the end plates.

Exit Ducts. Two exit ducts, both made of 0.93 inch outside diameter tubing, are used to carry the smoke downstream of the valve. One tube is approximately 2 feet 2 inches long with one $90^{\circ}$ bend and two $70^{\circ}$ bends. The second tube is 8 feet long with two $30^{\circ}$ bends. All bends have a 4 inch radius. Obviously, changing exit ducts (either in diameter or in length) will affect the exit flow characteristics of the smoke.

Valve. The critical element in the prototype design is the valve. For this application, the most important characteristic of the valve is the response

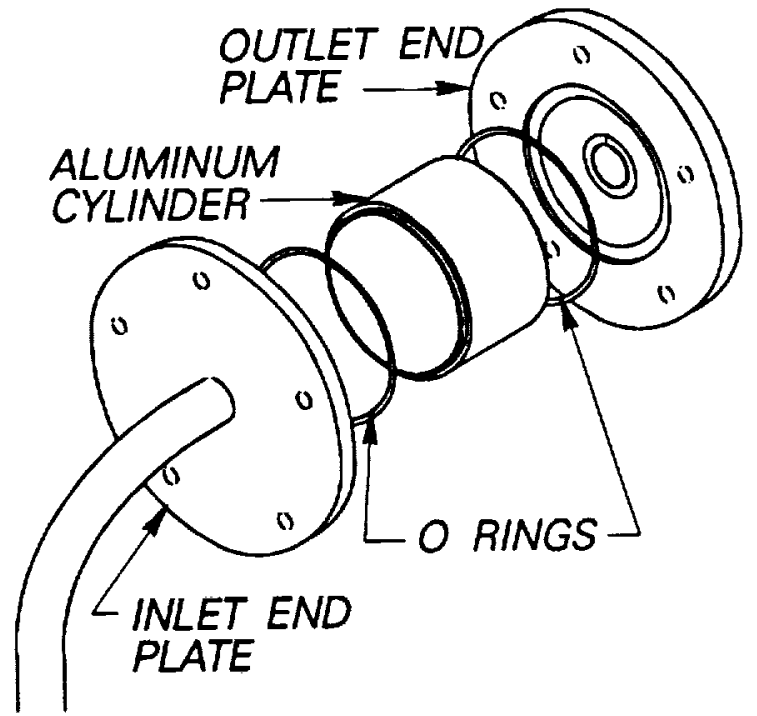

Figure 4. Plenum Chamber Subassembly time. The valve must respond quickly in order to produce smoke pulses with sharp edges. Also, in this prototype it was assumed that the valve had to operate reliably with no back pressure available to assist switching the valve position.

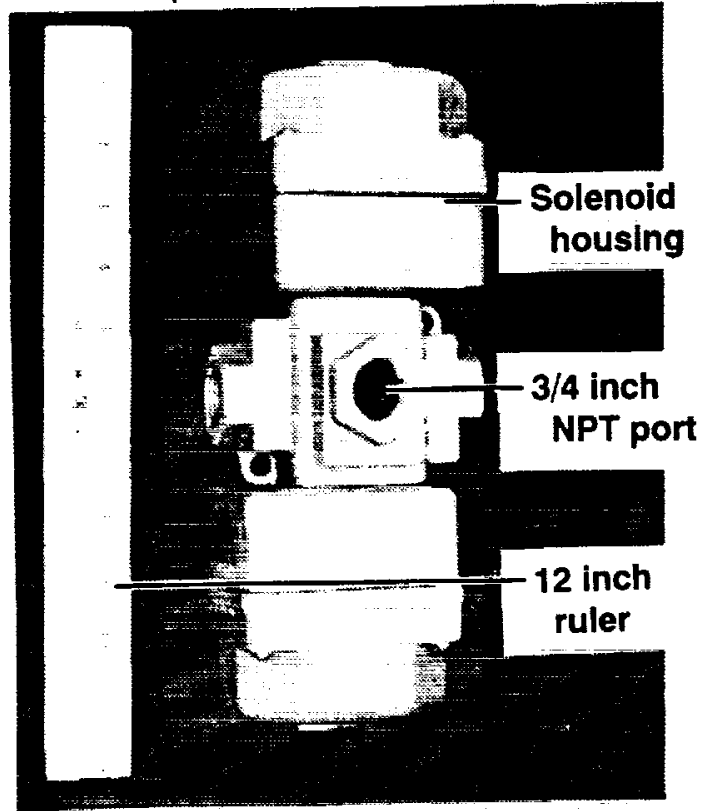

Figure 5. Three-way Double Solenoid Switching Valve

Two three-way valves were tested for this prototype design. Each is an ISI Fluid Power Detroit Line Air Valve for Industry design having $3 / 4$ inch NPT female ports. The original valve has a direct acting solenoid and a spring return. The second valve (Figure 5) has two direct acting solenoids. Both valves have a response time of 12 milliseconds, and operate at pressures up to $125 \mathrm{psig}$. The electrical power required by the valve is 115 Volt, $60 \mathrm{~Hz} \mathrm{AC}$. The solenoid draws 4.2 amps inrush and 0.6 amps holding. The manufacturer lists flow capacity $\left(C_{v}\right)$ at 5.7. The viton seals are designed for a temperature range of $40^{\circ} \mathrm{F}$ to $350^{\circ} \mathrm{F}^{15}$.

Materials. The cartridge container, container cap, plenum chamber, ring clamps, and pressure bearing tubing are aluminum 6061-T6. This material was chosen because of its machinability, weldability, and adequate strength at high temperatures. The cartridge retainer is an unknown alloy of aluminum. The tubing downstream of the valve is not pressure bearing so $6061-0$ aluminum was chosen for its ductility during bending. 
Silicone O-ring cord with a diameter of 1/8" was used to make O-rings; the ends were joined with silicone adhesive. The rupture disk is cut from 0.002 " brass shim stock; the rupture disk gaskets are cut from a $1 / 32^{\prime \prime}$ thick sheet of Garolite.

Determination of Wall Thicknesses. Because the system contains a gas mixture under pressure, wall thicknesses are important to guard against rupture. The important factors in determining the necessary thicknesses are cylinder radius (either inside or outside), pressures encountered, and strength of the material. For a cylinder with an inner pressure, the tangential stress $\sigma_{\theta}$ is tensile, with a maximum at the inner wall. This maximum tensile stress determines the needed thickness.

NASA has previously found the maximum temperature at

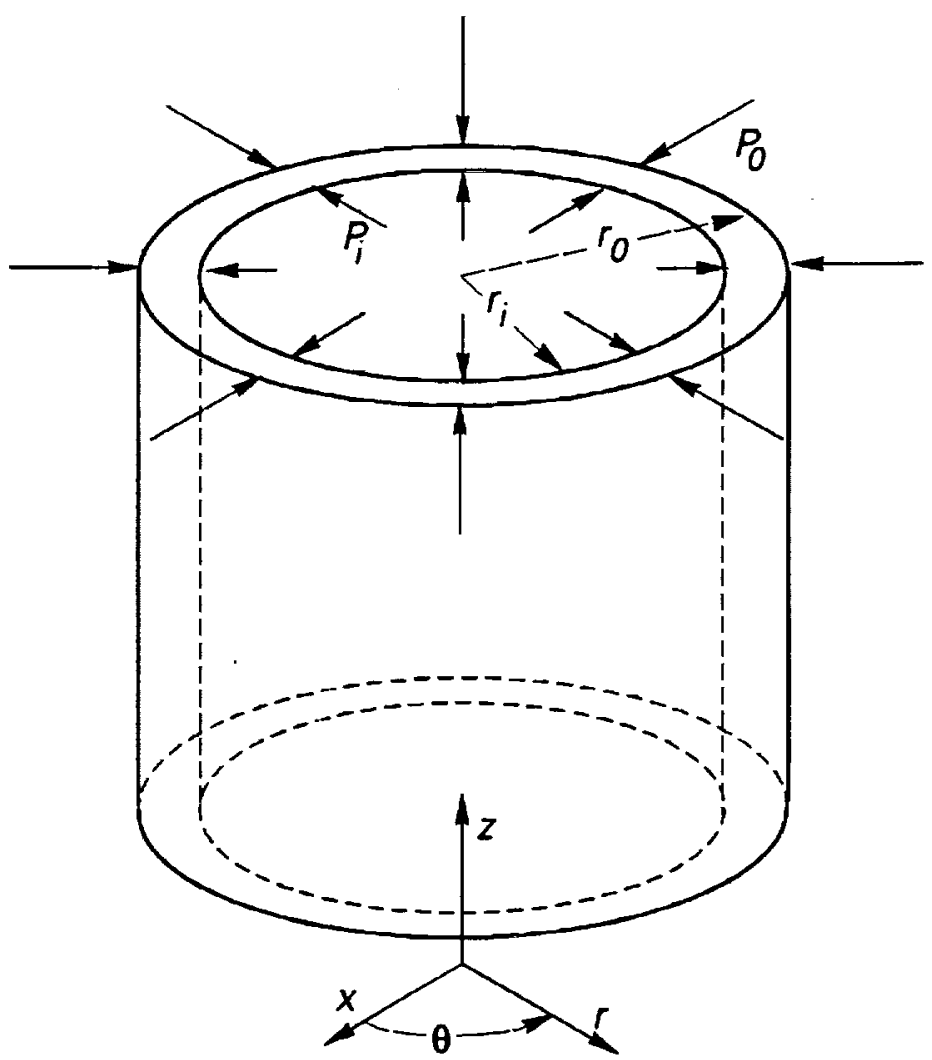

Figure 6. Stresses in a Cylinder Wall the wall in the cartridge container to be about $230^{\circ} \mathrm{F}$, using a resistance thermometer. The maximum pressure in the system before the rupture disk fails is 75 psid. To ensure a reasonable safety margin, the cylinder thicknesses were sized for a 10 hour exposure to $500^{\circ} \mathrm{F}$, and an internal pressure of $150 \mathrm{psid}$. Aluminum 6061-T6 has a tensile yield stress of $35,000 \mathrm{psi}$ at standard room temperature. After being exposed to $500^{\circ} \mathrm{F}$ for 10 hours, it retains $35 \%$ of its tensile strength.

The tangential stress $\sigma_{\theta}$ at the inner wall of a cylinder is:

$$
\sigma_{\theta}=\frac{P_{i}\left[\left(\frac{r_{0}}{r_{i}}\right)^{2}+1\right]-2 P_{0}\left(\frac{r_{0}}{r_{i}}\right)^{2}}{\left(\frac{r_{0}}{r_{i}}\right)^{2}-1}
$$

Rearranging to solve for $r_{i_{\max }}: r_{i_{\max }}=r_{0} \sqrt{\frac{\sigma_{\theta_{\max }}+2 P_{0}-P_{i}}{P_{i}+\sigma_{\theta_{\max }}}}$

Minimum thickness corresponds to maximum inside radius; hence, where $r_{0}=\frac{O . D \text {. }}{2}$. 
Therefore, $t_{\min }=r_{0}-r_{i_{\max }}=r_{0}\left(1-\sqrt{\frac{\sigma_{\theta_{\max }}+2 P_{0}-P_{i}}{P_{i}+\sigma_{\theta_{\max }}}}\right)$

Table 2 shows the minimum and acTable 2. Tubing Thicknesses tual thicknesses for the different outside radii of cylinders used.

Fittings. To best duplicate the conditions of the NASA SGS, all tubing used to duct the smoke is $1.0^{\prime \prime}$ O.D. and $0.035^{\prime \prime}$ thick. The valve, however, has $3 / 4^{\prime \prime}$ NPT female fittings. Therefore, aluminum aircraft AN fittings were needed to connect the tubing to the valve. An AN819-16D coupling sleeve was welded on the end of each 1.0" O.D. tube. An AN818-16D coupling nut could then connect the tube to an AN816-16-12D flared tube and pipe thread nipple. The pipe thread could then be screwed into the valve port. The igniter wires are fed out of the cartridge container through a brass compression fitting screwed into a hole in the container cap. The hole was drilled and tapped for a 1/8" NPT fitting. The compression fitting accommodates a 1/8" O.D. shaft. Silicone adhesive is applied around the top of the compression fitting to reduce the possibility of a leak.

\section{Instrumentation}

To obtain dynamic measurements of the pressure and temperatures of the gaseous mixture in the system, a pressure transducer and thermocouples were needed.

Pressure Transducer. A Validyne P305D differential pressure transducer was connected to the inlet plate of the plenum chamber using a 8-3/16" long, 3/16" O.D. aluminum tube as a stand-off. The stand-off was necessary to prevent excessive temperature at the pressure transducer. The stand-off is connected to the plenum chamber by a $3 / 16^{\prime \prime}$ flared tube and $1 / 8$ " pipe thread nipple. A hole was drilled and tapped in the plenum inlet to accommodate the $1 / 8^{\text {n }}$ NPT nipple. The pressure transducer was fitted with a 50 psid diaphragm. The pressure transducer can respond to signals ranging from 0 to $200 \mathrm{~Hz}$; the output signal range is \pm 5 volts.

Thermocouples. Three thermocouples were used to measure the temperature of the gaseous mixture, and to estimate the heat lost to the surroundings. The thermocouples are Type J (Iron - Constantan) with 1/16" O.D. sheaths. One thermocouple is inserted through a brass compression fitting in the side of the cartridge container, and extends just above the top of the smoke cartridge. This thermocouple is ungrounded. Its time constant in air at room temperature and atmospheric pressure, moving 65 feet per second, is approximately 4 seconds. The second thermocouple is inserted into the plenum chamber through a compression fitting mounted on the inlet plate. The thermocouple extends approximately $1 / 4$ " inside the plenum. It is an exposed junction thermocouple with a time constant of approximately 0.45 seconds. The third 
thermocouple is inserted near the duct exit downstream of the valve. It is located in the center of the tube and is also an exposed junction thermocouple.

Fittings. Pipe thread couplings (AN910-1D) were used to connect the pressure transducer stand-off to the plenum chamber, and to connect the thermocouples to the cartridge container, plenum chamber, and duct exit. To connect the pressure transducer stand-off and the thermocouples for the cartridge container and plenum chamber, the couplings were first cut in half. One half-coupling was then welded in place for each fitting. The couplings cracked during welding, however. As a result, it was necessary to mill the couplings down to a height of approximately $1 / 8^{\prime \prime}$. The remaining parts of the couplings were used as a guide to drill and tap the cartridge container and plenum inlet with 1/8" NPT holes. To connect the duct exit thermocouple to the tubing, a pipe thread coupling (AN910-1D) was welded to the tubing. A 1/8" diameter hole was then drilled into the tube for insertion of the thermocouple.

\section{Data Collection}

A Data Translation DT2821-F-16SE analog to digital conversion (AD) board was used to collect data with an IBM compatible 286 personal computer. The AV board has 16 12-bit A/D channels; each channel has a range of volts. The outputs of the pressure transducer and three thermocouples were sampled on four channels at a sample rate of 1000 samples/second each, for sixty seconds. This allowed enough time for the cartridge to ignite and burn to completion. A computer program which utilized ATLAB, a software package also produced by Data Translation, was used to control the AD board, as well as to control the valve and ignition of the cartridge. The data was stored in buffers using direct memory access (DMA), allowing the AV board to return control to the computer so it could be used to control the valve. After the data collection was complete, data from the buffers were written to output files.

Signal Conditioning. The output of Type $\mathrm{J}$ thermocouples at a temperature $1300^{\circ} \mathrm{F}$ above reference (ambient in this case) temperature is only 39.4 millivolts. Therefore, considerable amplification was necessary to obtain decent resolution with the AVD board. The outputs of the thermocouples were amplified by a factor of approximately 250 using operational amplifiers. The output of the pressure transducer has a range of volts. To maximize resolution, the output was amplified by a factor of approximately 2.0.

A Validyne CD12 transducer indicator is used to supply both excitation voltage and signal conditioning for the pressure transducer measuring dynamic pressure of the exit smoke flow. The transducer indicator has a frequency response of 0 to $1000 \mathrm{~Hz}$ and its output range is \pm 10 volts ${ }^{18}$ so no additional amplification is needed.

Synchronizing Cartridge Ignition and Data Collection. Cartridge ignition and data collection are synchronized using one of the two DT2821-F-16SE D/A output channels and a solid state relay. Data collection begins when the ignition signal is sent from the D/A output. The output of the relay is connected in series with the igniter and a 28 volt DC power supply. The D/A output from the AVD board is connected to the input of the relay. When the D/A output is 0 volts, the relay is open; when the D/A output is 5 volts, 
the relay is closed and ignition occurs. The maximum response time for the relay is 0.2 milliseconds ${ }^{21}$.

\section{Valve Control}

The valves are controlled using the remaining D/A output channel and solid state relays. Control of the single solenoid valve is straightforward. A 5 volt signal from the D/A output causes a relay to energize the solenoid and a 0 volt signal removes deenergizes the solenoid. Control of the double solenoid valve is slightly more complicated and a logic circuit is needed to control two relays. A rise in the output voltage (from 0 to 5 volts) causes one relay to energize its solenoid for 20 milliseconds. A drop in D/A voltage (from 5 to 0 volts) causes the other relay to energize its solenoid for 20 milliseconds. The response time of each relay varies from 0 to 8.3 milliseconds (half of a $60 \mathrm{~Hz}$ cycle) depending on the phase of the line power at the time the relay is signalled 21 . This unknown response lag leads to uncertainty in exactly when a valve closes with this arrangement.

\section{PRESSURE ESTIMATION}

Safe use of the intermittent flow visualization system requires information concerning the pressure inside the system as the smoke is pulsed. An alternate flow path must be provided for the smoke if complete blockage causes any of the following to occur:

1) pressure rises which result in system failure

2) pressure rises which are unsafe

3) smoke exit velocities which are unacceptably high.

Since it was not feasible to obtain experimental data for all possible pulse rates, using all possible system internal volumes, a model was developed to simulate the pressure in the system. Experiments were then conducted to validate this mathematical model.

\section{Chemical Reaction}

To model the pressure in the system, the compounds present and the amount of each constituent, as well as their physical states, must be known. Thus, knowledge of the chemical reaction which produces the smoke is essential. The smoke cartridges contain $\mathbf{3 3 0}$ grams of a pyrotechnic mixture. The reactants in this mixture are given in Table 3. When a 28 volt potential is applied to the ignitor, it produces a flame in the smoke cartridge which starts a chemical reaction. In this reaction, sucrose (the fuel) and potassium chlorate (the oxidizer) react to produce water vapor $\left(\mathrm{H}_{2} \mathrm{O}\right)$, carbon dioxide $\left(\mathrm{CO}_{2}\right)$, carbon monoxide $(\mathrm{CO})$, and potassium chloride $(\mathrm{KCl})$. This reaction is exothermic and gives off heat which causes the terephthalic acid $\left(\mathrm{C}_{8} \mathrm{H}_{6} \mathrm{O}_{4}\right)$ to sublime. The liberation of gases inside the cartridge causes a pressure increase, forcing them out of the cartridge. Once away from the heat of the reaction, the gases cool. The terephthalic acid recondenses to form visible, solid particles when it reaches a temperature of $572^{\circ} \mathrm{F}$. 
Table 3. Smoke Cartridge Reactants

\begin{tabular}{|c|c|c|c|c||}
\hline reactant & chemical formula & $\%$ by weight & mass (grams) & moles (g-moles) \\
\hline $\begin{array}{c}\text { terephthalic } \\
\text { acid }\end{array}$ & $\mathrm{C}_{8} \mathrm{H}_{6} \mathrm{O}_{4}$ & 57 & 188 & 1.130 \\
\hline $\begin{array}{c}\text { potassium } \\
\text { chlorate }\end{array}$ & $\mathrm{KClO}_{3}$ & 23 & 76 & 0.619 \\
\hline sucrose & $\mathrm{C}_{12} \mathrm{H}_{22} \mathrm{O}_{11}$ & 14 & 46 & 0.135 \\
\hline $\begin{array}{c}\text { magnesium } \\
\text { carbonate }\end{array}$ & $\mathrm{MgCO}_{3}$ & 3 & 10 & 0.117 \\
\hline nitrocellulose & (compound) & 2 & 7 & (compound) \\
\hline graphite & $\mathrm{C}$ & 1 & 3 & 0.583 \\
\hline
\end{tabular}

The reaction of sucrose with $\mathrm{KClO}_{3}$ occurs in two steps. $\mathrm{KClO} 3$ decomposes exothermically to form $\mathrm{KCl}$ and $\mathrm{O}_{2}$ :

$$
2 \mathrm{KClO}_{3} \rightarrow 2 \mathrm{KClO}_{3}+3 \mathrm{O}_{2}+\text { heat }
$$

Therefore, $0.619 \mathrm{~g}$-moles of $\mathrm{KClO}_{3}$ produces $0.619 \mathrm{~g}$-moles of $\mathrm{KCl}$ and $0.9285 \mathrm{~g}$-moles of $\mathrm{O}_{2}$. The sucrose then reacts with the oxygen to produce $\mathrm{H}_{2} \mathrm{O}, \mathrm{CO}_{2}$, and $\mathrm{CO}$ :

$$
1350 \mathrm{C}_{12} \mathrm{H}_{22} \mathrm{O}_{11}+9285 \mathrm{O}_{2} \rightarrow 14850 \mathrm{H}_{2} \mathrm{O}+13830 \mathrm{CO}+2370 \mathrm{CO}_{2}
$$

Table 4. Products of Sucrose- $\mathrm{KClO}_{3}$ Reaction and Decomposition of $\mathrm{MgCO}_{3}$

\begin{tabular}{|c|c|c|c||}
\hline compound & mass (grams) & g-moles & state \\
\hline $\mathrm{H}_{2} \mathrm{O}$ & 26.8 & 1.485 & gas \\
\hline $\mathrm{CO}$ & 38.7 & 1.383 & gas \\
\hline $\mathrm{CO}_{2}$ & 15.6 & 0.354 & gas \\
\hline $\mathrm{KCl}$ & 46.2 & 0.619 & solid \\
\hline $\mathrm{MgO}$ & 4.72 & 0.117 & solid \\
\hline
\end{tabular}

The complete reaction of $0.135 \mathrm{~g}$-moles of sucrose with $0.619 \mathrm{~g}$-moles of $\mathrm{KClO}_{3}$ is:

$0.135 \mathrm{C}_{12} \mathrm{H}_{22} \mathrm{O}_{11}+0.619 \mathrm{KClO}_{3} \rightarrow 0.619 \mathrm{KCl}+1.485 \mathrm{H}_{2} \mathrm{O}+1.3830 \mathrm{CO}+0.237 \mathrm{CO}_{2}$ The $\mathrm{MgCO}_{3}$ decomposes to form $\mathrm{MgO}$ and $\mathrm{CO}_{2} 22$ :

$$
0.117 \mathrm{MgCO}_{3} \rightarrow 0.117 \mathrm{MgO}+0.117 \mathrm{CO}_{2}
$$

The products of the reaction of sucrose with $\mathrm{KClO}_{3}$ and the decomposition of $\mathrm{MgCO}_{3}$ are given in Table 4 .

The contribution of the decomposition of nitrocellulose to the reaction products is more difficult to determine. Nitrocellulose is not a single compound, but a group of compounds formed by the reaction of cellulose with concentrated nitric acid 23 . Therefore, reactions involving it are difficult to determine. However, it makes up a small part ( $2 \%$ by weight) of the chemical mixture. As a simplification, the decomposition of nitrocellulose was assumed to add mass to the gaseous products, but not change their rel- 
ative proportions. Some nitrogen gas $\left(\mathrm{N}_{2}\right)$ is given off, but in negligible amounts. The products of the chemical reactions in the smoke cartridge are given in Table 5 and the important physical properties of these products are given in Table 6.

Table 5. Products of Smoke Cartridge Reaction

\begin{tabular}{|c|c|c|c|}
\hline compound & mass (grams) & g-moles & state \\
\hline $\mathrm{H}_{2} \mathrm{O}$ & 29.0 & 1.61 & gas \\
\hline $\mathrm{CO}$ & 41.9 & 1.50 & gas \\
\hline $\mathrm{CO}_{2}$ & 16.8 & 0.38 & gas \\
\hline $\mathrm{C}_{8} \mathrm{H}_{6} \mathrm{O}_{4}$ & 188.0 & 1.13 & gas \\
\hline $\mathrm{KCl}$ & 46.2 & 0.619 & solid \\
\hline $\mathrm{MgO}$ & 4.7 & 0.117 & solid \\
\hline $\mathrm{C}$ & 3.3 & 0.583 & solid \\
\hline
\end{tabular}

Table 6. Physical Properties of Smoke Cartridge Products

\begin{tabular}{|c|c|c|c||}
\hline Compound & $\begin{array}{c}\text { Molecular } \\
\text { Weight }\end{array}$ & $\begin{array}{c}\text { Melting Point } \\
\left({ }^{\circ} \mathrm{C}\right)\end{array}$ & $\begin{array}{c}\text { Boiling Point } \\
\left({ }^{\circ} \mathrm{C}\right)\end{array}$ \\
\hline $\mathrm{H}_{2} \mathrm{O}$ & 18.0153 & 0 & 100 \\
\hline $\mathrm{CO}$ & 28.01 & -199 & -191.5 \\
\hline $\mathrm{CO}_{2}$ & 44.01 & --- & $-78.5^{*}$ \\
\hline $\mathrm{C}_{8} \mathrm{H}_{6} \mathrm{O}_{4}$ & 166.14 & --- & $>300^{*}$ \\
\hline $\mathrm{KCl}$ & 74.56 & 770 & $1500^{*}$ \\
\hline $\mathrm{MgO}$ & 40.31 & 2852 & 3600 \\
\hline $\mathrm{C}$ & 12.0112 & $? ? ? ?$ & $? ? ? ?$ \\
\hline
\end{tabular}

Note: * Denotes sublimation.

\section{Adiabatic Flame Temperature of Reaction}

An estimate of the temperature of the reaction products is needed to determine the physical states of the different products. An estimate of the upper limit of this temperature is the adiabatic flame temperature. The adiabatic flame temperature can be found using the conservation of energy. For an open system at steady state conditions with negligible changes in kinetic and potential energies, the heat put into the system equals the shaft work done by the system plus the change in total enthalpy of the system:

$$
Q=W_{s}+H_{P}-H_{R}
$$

The total enthalpy of an ideal gas mixture can be written as the sum of the enthalpy of formation at some arbitrary reference state, plus the change in enthalpy caused by the mixture being at a temperature other than the reference state:

$$
H=\sum N_{i}\left[\Delta \bar{h}_{f_{i}}^{\circ}+\Delta \bar{h}_{i}\left(T-T_{\text {ref }}\right)\right]
$$


Substituting this total enthalpy into the conservation of energy equation gives:

$$
Q=W_{s}+\sum_{P} N_{i}\left[\Delta \bar{h}_{f_{i}}^{\circ}+\Delta \bar{h}_{i}\left(T-T_{\text {ref }}\right)\right]-\sum_{R} N_{i}\left[\Delta \bar{h}_{f_{i}}^{\circ}+\Delta \bar{h}_{i}\left(T-T_{\text {ref }}\right)\right]
$$

For an adiabatic process with no work done by the system, and negligible changes in kinetic and potential energy, this reduces to:

$$
\sum_{P} N_{i}\left[\Delta \bar{h}_{f_{i}}^{\circ}+\Delta \bar{h}_{i}\left(T-T_{\text {ref }}\right)\right]=\sum_{R} N_{i}\left[\Delta \bar{h}_{f_{i}}^{\circ}+\Delta \bar{h}_{i}\left(T-T_{\text {ref }}\right)\right]
$$

This result gives the maximum temperature of the reaction products 24 .

As a simplifying assumption, only the reaction of sucrose with $\mathrm{KClO}_{3}$ and the sublimation of terephthalic acid will be considered. This assumption is reasonable because these three compounds constitute $94 \%$ of the pyrotechnic mixture. The reaction considered is:

$$
\begin{gathered}
0.135 \mathrm{C}_{12} \mathrm{H}_{22} \mathrm{O}_{11}(\mathrm{~s})+0.619 \mathrm{KClO}_{3}(\mathrm{~s})+1.13 \mathrm{C}_{8} \mathrm{H}_{6} \mathrm{O}_{4}(\mathrm{~s}) \rightarrow \\
0.619 \mathrm{KCl}(\mathrm{s})+1.485 \mathrm{H}_{2} \mathrm{O}(\mathrm{g})+0.237 \mathrm{CO}_{2}(\mathrm{~g})+1.13 \mathrm{C}_{8} \mathrm{H}_{6} \mathrm{O}_{4}(\mathrm{~g})
\end{gathered}
$$

The reactants are initially assumed to be at $25^{\circ} \mathrm{C}$, therefore $\Delta \bar{h}_{i}\left(T-T_{\text {ref }}\right)$ for the reactants is zero.

Because no data giving the enthalpy of $\mathrm{KCl}$ at states other than the reference state $\left(25^{\circ} \mathrm{C}\right)$ are readily available, it is assumed that $\mathrm{KCl}$ remains at the reference state. This assumption will artificially inflate the adiabatic flame temperature, which is already known to be greater than the actual flame temperature. Thus, the prediction should be conservative.

Table 7. Heat of Formation of the Reactants

\begin{tabular}{|c|c|c||}
\hline Reactant & Number of g-moles & $\Delta \bar{h}_{f_{j}}^{0}(\mathrm{~kJ} / \mathrm{g}$-mole $)$ \\
\hline $\mathrm{C}_{12} \mathrm{H}_{22} \mathrm{O}_{11}$ & 0.135 & -2227.4 \\
\hline $\mathrm{KClO}_{3}$ & 0.619 & -398 \\
\hline $\mathrm{C}_{8} \mathrm{H}_{6} \mathrm{O}_{4}$ & 1.13 & -806 \\
\hline
\end{tabular}

Table 8. Reaction Products' Heat of Formation and Change in Enthalpy for a Temperature of $886^{\circ} \mathrm{K}$

\begin{tabular}{|c|c|c|c||}
\hline Product & $\begin{array}{c}\text { Number of } \\
\text { g-moles }\end{array}$ & $\begin{array}{c}\Delta \bar{h}_{f_{i}}^{\circ} \\
(\mathrm{kJ} / \mathrm{g} \text {-mole })\end{array}$ & $\begin{array}{c}\Delta \bar{h}_{i}\left(T-T_{\text {ref }}\right) \\
(\mathrm{kJ} / \mathrm{g} \text {-mole })\end{array}$ \\
\hline \hline $\mathrm{KCl}$ & 0.619 & -437 & -- \\
\hline $\mathrm{H}_{2} \mathrm{O}$ & 1.485 & -241 & 21.53 \\
\hline $\mathrm{CO}$ & 1.383 & -111 & 18.07 \\
\hline $\mathrm{CO}_{2}$ & 0.237 & -394 & 27.52 \\
\hline $\mathrm{C}_{8} \mathrm{H}_{6} \mathrm{O}_{4}$ & 1.13 & -718 & 133.51 \\
\hline
\end{tabular}


The temperature of the products at which the sums of the enthalpies of the products and reactants are equal is $886^{\circ} \mathrm{K}$ or $613^{\circ} \mathrm{C}$. Table 7 gives the number of moles present and the heat of formation at the reference state of each reactant. Table 8 gives the number of moles present, the heat of formation at the reference state, and the change in enthalpy for a temperature of $886^{\circ} \mathrm{K}$ for each product 24,25 .

Table 6 shows that water, carbon monoxide, carbon dioxide, and terephthalic acid are in the gaseous state at $613^{\circ} \mathrm{C}$, while potassium chloride and magnesium oxide remain in the solid state; hence,the initial assumptions concerning the physical states of the reaction products are correct. It follows that the gaseous mixture in the smoke generating system contains only water vapor, carbon monoxide, carbon dioxide, and terephthalic acid.

Thermodynamlc Properties of Terephthalic Acid

The enthalpy of tereph-

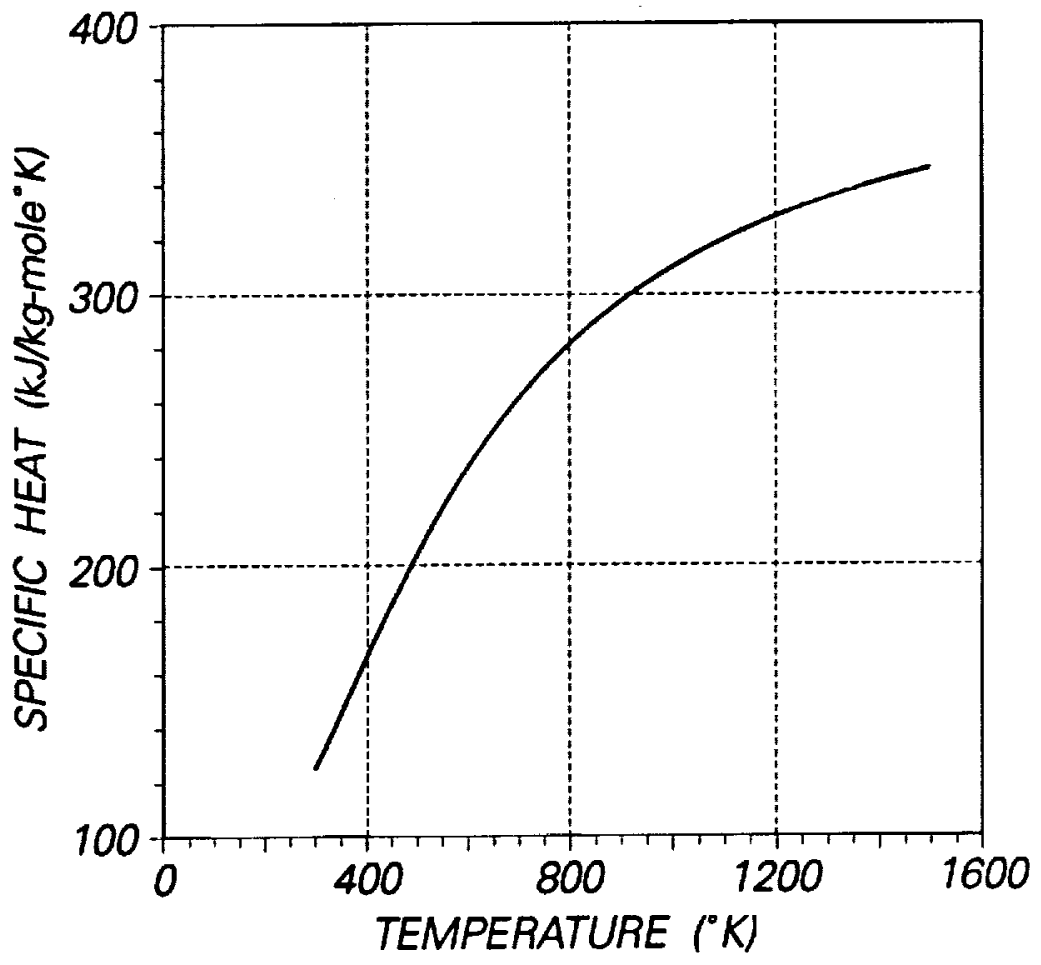

Figure 7. Ideal Gas Heat Capacity of Terephthalic Acid thalic acid can be found using a method given by Daubert and Danner 25 . The ideal gas heat capacity, shown in Figure 7, is:

$$
C_{P}^{0}=A+B \exp \left(\frac{-C}{T^{D}}\right)
$$

where $A=97000, B=293800, C=27620, D=1.6442$, and $T$ is the temperature in ${ }^{\circ} \mathrm{K}$ and the units for ideal gas heat capacity are $\mathrm{J} / \mathrm{kg}$-mole ${ }^{\circ} \mathrm{K}$. These coefficients are valid for temperatures ranging from $100^{\circ} \mathrm{K}$ to $1500^{\circ} \mathrm{K}$. Assuming the terephthalic acid behaves as an ideal gas, the change in enthalpy due to a change in temperature can be found by integrating the ideal gas heat capacity with respect to temperature:

$$
\Delta H=\int_{T_{1}}^{T_{2}} C_{P}^{0}
$$

The ideal gas heat capacity with the exponential expanded in series form is:

$$
C_{P}^{0}=97000+293800\left(1-\frac{27620}{T^{1.6442}}+\frac{27620^{2}}{2 ! T^{2(1.6442)}}-\frac{27620^{3}}{3 ! T^{3(1.6442)}}+\ldots\right)
$$

Or, alternatively: 


$$
C_{P}^{0}=97000+293800 \sum\left[(-1)^{m} \frac{27620^{m}}{m ! T^{1.6442 m}}\right]
$$

Term by term integration leads to:

$$
\Delta H=\int_{T_{1}}^{T_{2}} C_{P}^{0} d T=\left.(97000+293800) T \sum_{m}\left((-1)^{m} \frac{\left.27620^{m} T^{(1-1.6442 m}\right)}{m !(1-1.6442 m)}\right)\right|_{T_{1}} ^{T_{2}}
$$

The summation is carried out until the last term alters the sum less than $0.01 \%$. The enthalpy change due to a change in temperature is called the sensible enthalpy change. The summation of the sensible enthalpy change with the heat of formation or enthalpy due to the chemical makeup of the gas gives the total enthalpy for any temperature.

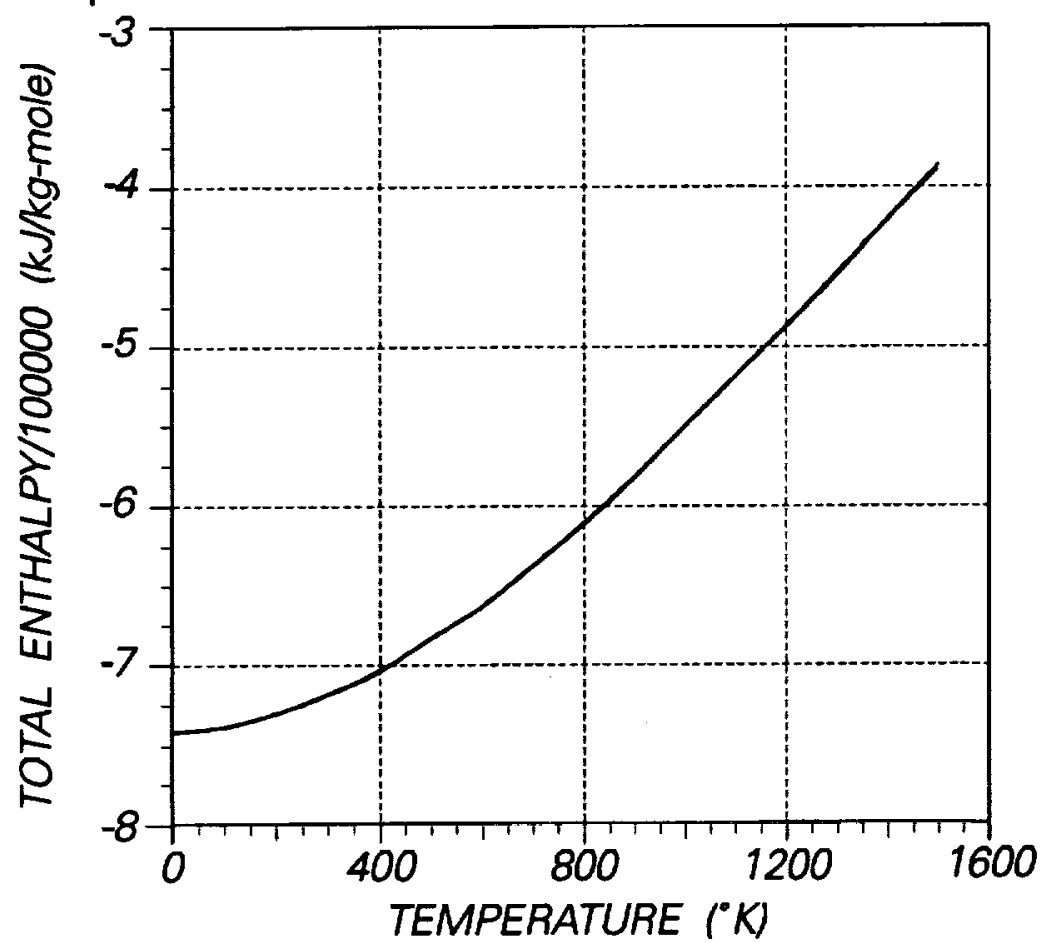

Figure 8. Ideal Gas Total Enthalpy of Terephthalic Acid
The heat of formation of terephthalic acid at $298{ }^{\circ} \mathrm{K}$ and one atmosphere is $717,890 \mathrm{~kJ} / \mathrm{kg}$-mole. The ideal gas total enthalpy for terephthalic acid (Figure 8 ) is approximated as a third order polynomial curve. The heat of formation is based on an arbitrary reference state in which elements in their natural, stable state have an enthalpy of 0 at one atmosphere and $25{ }^{\circ} \mathrm{C}$. However, enthalpy as used in the energy equation, generally is zero at $0^{\circ} \mathrm{K}$. To obtain an ideal gas enthalpy for terephthalic acid for use in the energy equation, $741,088 \mathrm{~kJ} / \mathrm{kg}$ mole must be added to the heat of formation plus the sensible enthalpy change so that the enthalpy equals zero at $0^{\circ} \mathrm{K}$. The enthalpy of terephthalic acid with a value of zero at the reference state $\left(0^{\circ} \mathrm{K}\right)$ is shown in Figure 9.

\section{Mixture Properties}

The properties of a mixture of ideal gases are determined by its composition and temperature. The composition of the smoke mixture is given in Table 9, along with the critical temperature and pressure of each component. Since the pressure in the system 
will be much lower than the critical pressure of all of the mixture components, the mixture can be assumed to behave as an ideal gas.

The molecular weight of a mixture of ideal gases is equal to the mass of the mixture divided by the number of moles. The mixture molecular weight is $M_{m}=59.7$ atomic mass units (amu). The gas constant of a mixture of ideal gases can be found by dividing the universal gas constant by the molecular weight. The gas constant of the mixture is $R_{m}=$ $0.139 \mathrm{~kJ} / \mathrm{kg}^{\circ} \mathrm{K}$.

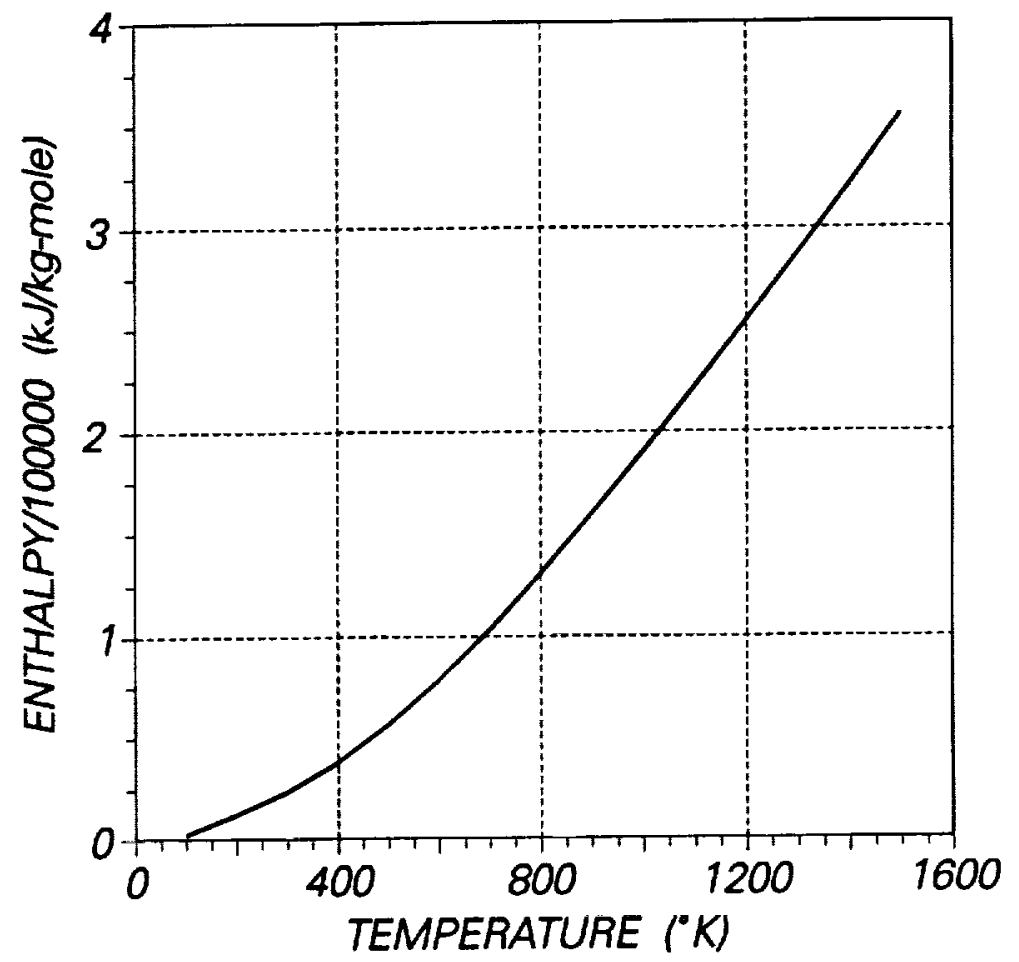

Figure 9. Enthalpy of Terephthalic Acid for Use in Conservation of Energy

Table 9. Mixture Composition

\begin{tabular}{|c|c|c|c||c|}
\hline Component & $\begin{array}{c}\text { Mass } \\
(\text { grams })\end{array}$ & $\begin{array}{c}\text { Weight } \\
(\%)\end{array}$ & $\begin{array}{c}\text { Critical Temperature } \\
\left({ }^{\circ} \mathrm{K}\right)\end{array}$ & $\begin{array}{c}\text { Critical Pressure } \\
(\mathrm{psi})\end{array}$ \\
\hline $\mathrm{C}_{8} \mathrm{H}_{6} \mathrm{O}_{4}$ & 188 & 68 & 1390 & 573 \\
\hline $\mathrm{CO}$ & 41.9 & 1541 & 133 & 508 \\
\hline $\mathrm{H}_{2} \mathrm{O}$ & 29 & 11 & 647 & 18.03204 \\
\hline $\mathrm{CO}_{2}$ & 16.8 & 6 & 304.2 & 1072 \\
\hline
\end{tabular}

The specific enthalpy of a mixture of ideal gases is dependent on the temperature and composition of the mixture. The specific enthalpy at any temperature can be found by summing the contribution of each of the mixture components. The specific enthalpy on a mass basis is given by:

$$
h_{m}=\sum_{i} m f_{i} h_{i}
$$

where $m f_{i}$ is the mass fraction of the ith component (the mass of the ith component divided by the total mass). The specific enthalpy on a molar basis is given by:

$$
\bar{h}_{m}=\sum_{i} y_{i} \bar{h}_{i}
$$


where $y_{i}$ is the mole fraction of the ith component (the number of moles of the ith component divided by the total number of moles).

Table 10. Mass Fractions and Mole Fractions for the Smoke Mixture Compounds

\begin{tabular}{|c|c||c|}
\hline Compound & Mass Fraction & Mole Fraction \\
\hline $\mathrm{C}_{8} \mathrm{H}_{6} \mathrm{O}_{4}$ & 0.682 & 0.245 \\
\hline $\mathrm{CO}$ & 0.152 & 0.325 \\
\hline $\mathrm{H}_{2} \mathrm{O}$ & 0.105 & 0.348 \\
\hline $\mathrm{CO}_{2}$ & 0.061 & 0.083 \\
\hline
\end{tabular}

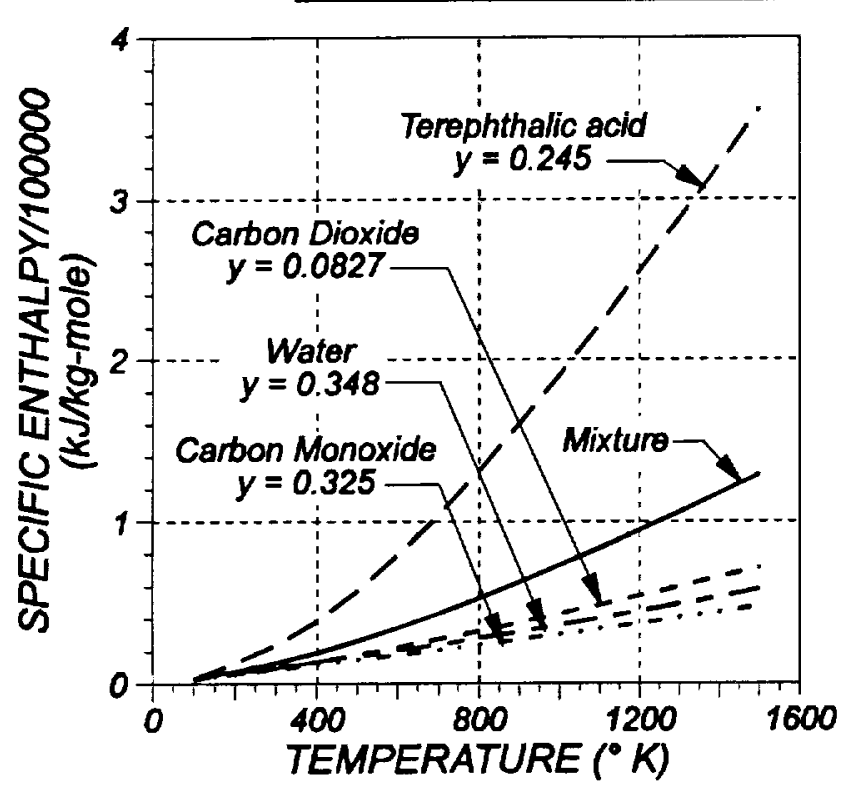

Figure 10. Specific Enthalpy of Terephthalic Acid Used in Conservation of Energy

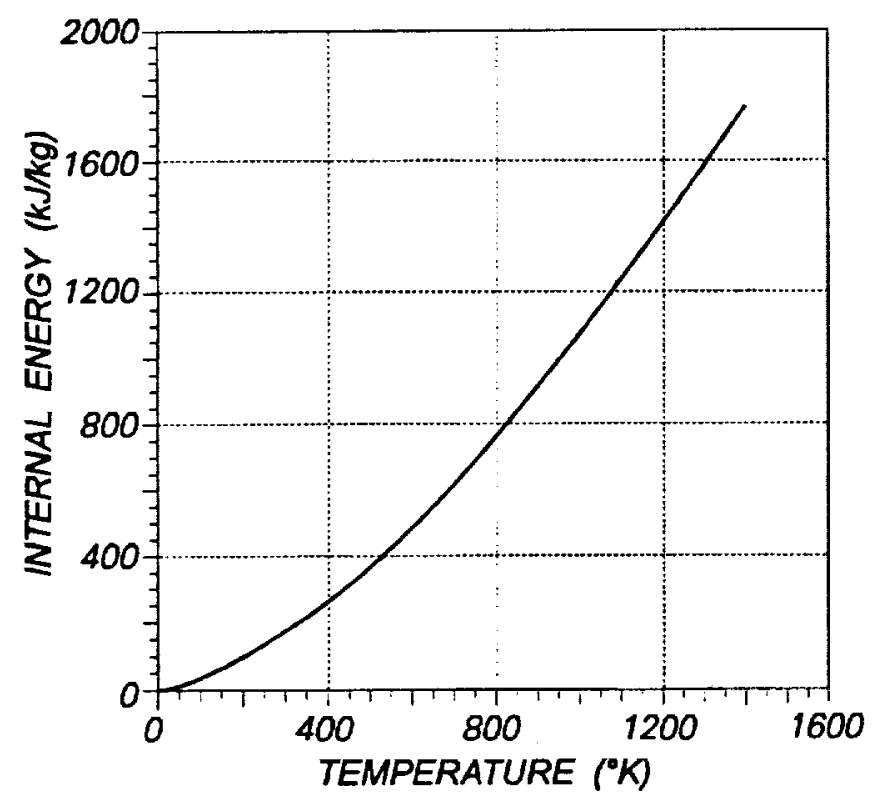

Figure 11. Internal Energy of the Mixture
The specific enthalpies of $\mathrm{CO}, \mathrm{H}_{2} \mathrm{O}$, and $\mathrm{CO}_{2}$ can be found in tables in most introductory thermodynamics texts. The specific enthalpy of terephthalic acid was discussed earlier. The mass fractions and mole fractions of the smoke mixture are given in Table 10. The specific enthalpy on a molar basis for the mixture, as well as each component, is shown in Figure 10. The enthalpy of the mixture on a mass basis is the enthalpy on a molar basis divided by the molecular weight, $59.7 \mathrm{amu}$.

Another important thermodynamic property is internal energy, which property can be found from enthalpy, the ideal gas constant, and temperature:

$$
h_{m}=u_{m}+R_{m} T
$$

The internal energy on a mass basis for the gaseous mixture is shown in Figure 11.

\section{Pressure Rise and Exit Velocity Es- timation}

The laws of conservation of mass and energy applied to the control volume shown in Figure 12, along with simplifying assumptions which will be discussed shortly, can be used to estimate the pressure buildup in the system caused by shutting off the flow of smoke. The law of conservation of mass can be expressed as: 


$$
\int_{A_{i}} \rho V_{n} d A-\int_{A_{\theta}} \rho V_{n} d A=\frac{d}{d t} \int_{v} \rho d v
$$

Conservation of energy gives:

$$
\begin{aligned}
& Q=W+\int_{A_{\theta}}\left(h+\theta_{p}+\theta_{k}\right) \rho V_{n} d A- \\
& \int_{A_{i}}\left(h+\theta_{p}+e_{k}\right) \rho V_{n} d A+\frac{d}{d t} \int_{V} e \rho d v
\end{aligned}
$$

The simplifying assumptions are:

(1) The mixture inside the system behaves as an ideal gas.

(2) Thermodynamic properties are not a function of the location inside the system (there is

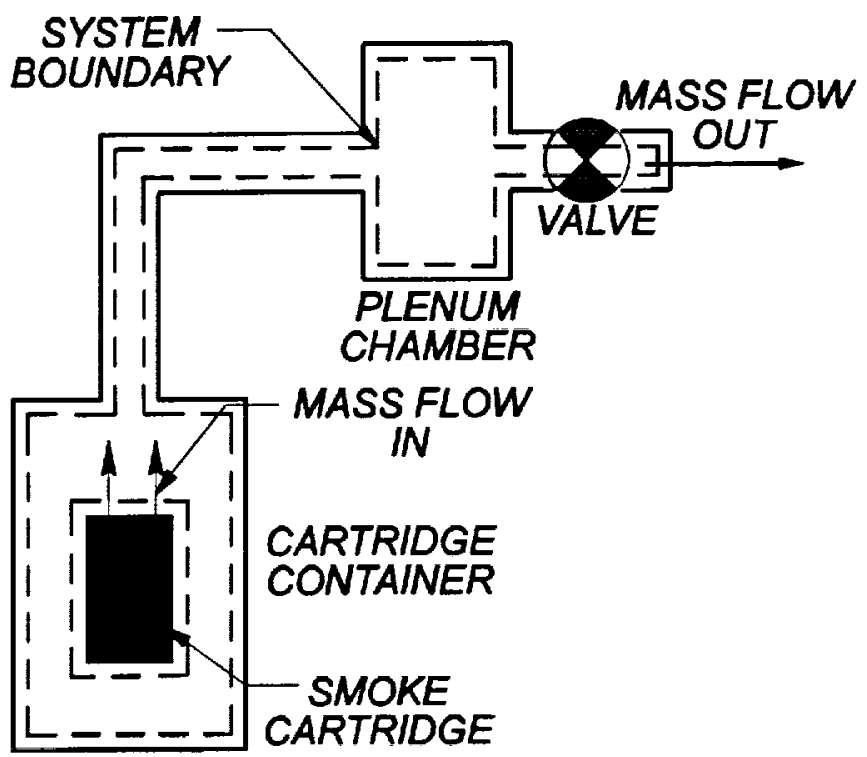

Figure 12. Thermodynamic Control Volume complete mixing).

(3) The flow through the system inlets and exits is uniform.

(4) The kinetic and potential energies of the mixture are negligible compared to the enthalpy.

(5) No shaft work is done by the system.

(6) The rate of energy entering the system by heat transfer is constant and can be found from the initial conditions.

(7) The enthalpy of the mixture can be found as outlined previously.

(8) The pressure losses in the exit duct are negligible.

(9) The valve's flow capacity is a linear function of time as it opens and closes.

(10) The mass flow rate through the valve can be found using available gas flow graphs ${ }^{26}$.

(11) The smoke cartridge burns at a constant rate.

Using assumptions 2 through 5 and rearranging, conservation of mass reduces to:

$$
\dot{m}_{\text {sys }}=\sum_{\text {inlet }} m-\sum_{\text {exit }} m
$$

Similarly, conservation of energy reduces to:

$$
\dot{E}_{\text {sys }}=Q+\sum_{\text {inlet }} \dot{m} h-\sum_{\text {exit }} m h,
$$

where the system energy $\left(E_{s y s}\right)$ is the specific internal energy multiplied by the mass in the system: $E_{\text {sys }}=\rho u^{i v}$

Once the initial conditions have been determined, the two conservation equations (mass and energy) can be integrated numerically. The initial conditions are found by measuring the pressure and temperature in the system and the temperature of the mass entering the system. It is assumed that the system has initially reached a steady 
state condition. This assumption requires a constant burn rate in the smoke cartridge. Although the burn rate is not perfectly constant, the mass flow rate into the system changes slowly compared to the mass flow rate out of the system as the valve closes and opens. The mass flow rate out of the system can be found using

$$
S F C H=C_{V} F_{G} F_{S G} F_{T}
$$

$F_{G}$ is obtained using gas flow graphs (Figures 13 and 14). The valve inlet pressure, as well as the pressure drop across the valve, equals the differential pressure between the inside and outside of the plenum chamber. $C_{V}$ is the valve flow capacity, a constant for the valve provided by the manufacturer. $F_{S G}$ is the specific gravity correction factor for the gaseous mixture, defined as

$$
F_{S G}=\frac{1}{\sqrt{S G}}
$$

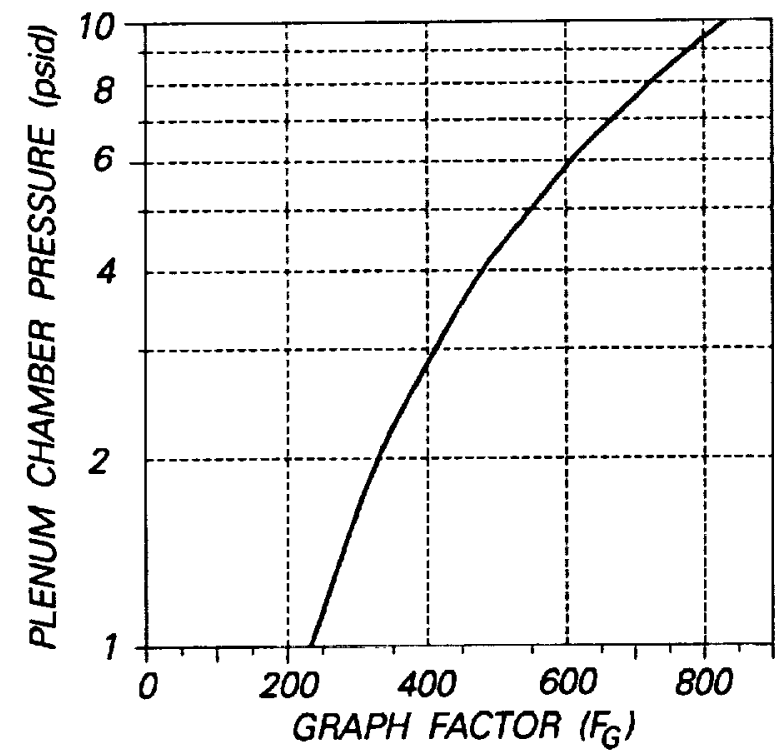

Figure 13. Gas Flow Graph for Low Pressure Drop Across Valve

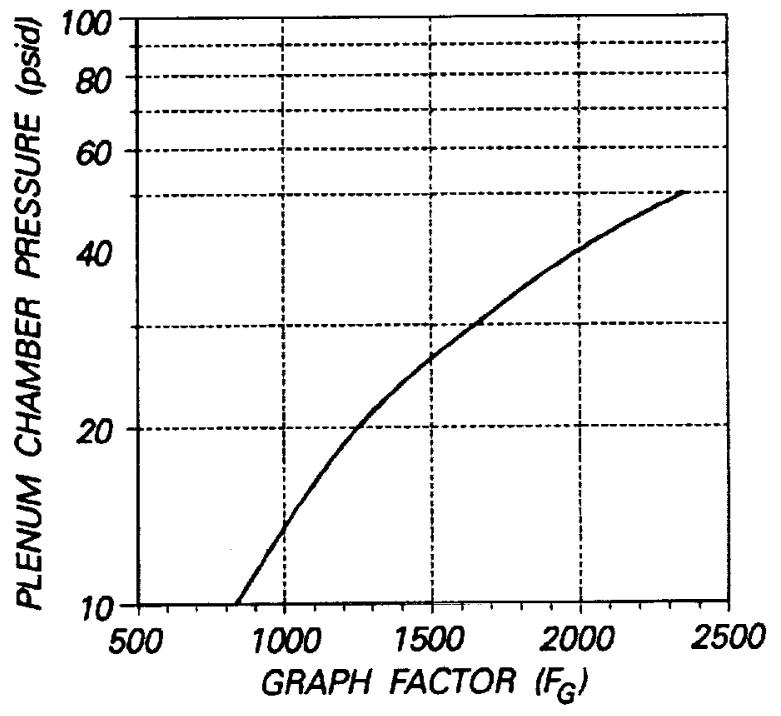

Figure 14. Gas Flow Graph for High Pressure Drop Across Valve

$F_{T}$ is a temperature correction factor defined as:

$$
F_{T}=\sqrt{\frac{530}{460+{ }^{\circ} \mathrm{F}}}
$$

SCFH is the standard cubic feet per hour of gas flowing through the valve. A standard cubic foot of gas is one cubic foot of gas at $14.7 \mathrm{psia}$ and $70^{\circ} \mathrm{F}$. The mass flow rate through the valve can be found by multiplying the SCFH by the density of the gas at standard conditions.

Since the flow is assumed to be steady initially, the mass flow rate of smoke into the system is the same as the initial mass flow rate out of the system. The enthalpy of the mass entering the system can be found by measuring the temperature of the mass entering the system, and the enthalpy of the mass leaving the system can be found by measuring the temperature of the mass in the plenum chamber. The assumption of an initial steady state allows the rate of energy leaving the system by heat transfer to be found. The initial mass in the system can be determined from the pressure, the tem- 
perature, and the volume; the initial total system energy is obtained from the initial mass and the temperature.

The valve flow capacity is assumed to change linearly with time as it closes or opens. The gas flow graphs give the mass flow rate through the valve as it cycles.

The exit velocity of the smoke is calculated by dividing the mass flow rate through the valve by the density of the smoke times the exit area. The density of the exit smoke is determined by the ambient pressure and the exit temperature of the smoke.

\section{TEST RESULTS}

\section{Experimental Procedure}

Ten experiments were conducted to obtain the data needed to determine the pressure rise estimation initial conditions, and to verify the pressure rise and exit velocity estimations. The first experiment was used to ensure that the pressure transducer stand-off would keep the temperature at the pressure transducer from rising above the recommended level, and that no residue would contaminate the pressure transducer. The next six experiments were used to work out problems involving data collection, valve reliability, and valve control. The last three experiments validated the pressure rise and exit velocity predictions for different system volumes.

During the test firings, two different valves were tested. One valve has a single solenoid with a spring return. The second valve has two solenoids. Two methods of pulsing the smoke were also employed. In the first method, the flow of smoke was repeatedly switched between two ports. One exit port was connected to an exit duct that was 2 feet 2 inches long, and the other to a duct that was 8 feet long. In the second method, the flow of smoke was repeatedly blocked for short periods of time; the exit port was again connected to the exit duct that was 2 feet 2 inches long. Table 11 summarizes the system parameters for each test.

Table 11. Test Configurations

\begin{tabular}{|c|c|c|c||}
\hline Test Number & Valve Used & Method of Pulsing & System Volume (in. ${ }^{3}$ ) \\
\hline 1 & Single Solenoid & Switching Ports & 100.7 \\
\hline 2 & Single Solenoid & Switching Ports & 100.7 \\
\hline 3 & Single Solenoid & Switching Ports & 100.7 \\
\hline 4 & Single Solenoid & Switching Ports & 100.7 \\
\hline 5 & Single Solenoid & Switching Ports & 100.7 \\
\hline 6 & Single Solenoid & Blocking Path & 100.7 \\
\hline 7 & Double Solenoid & Blocking Path & 100.7 \\
\hline 8 & Double Solenoid & Blocking Path & 100.7 \\
\hline 9 & Double Solenoid & Blocking Path & 125.7 \\
\hline 10 & Double Solenoid & Blocking Path & 175.7 \\
\hline
\end{tabular}


During firings 2 through 8 , measurements of differential pressure between the inside and outside of the plenum chamber, and temperatures at the smoke cartridge exit, in the plenum chamber, and at the duct exit were taken. In tests 9 and 10, dynamic pressure at the duct exit was measured using a pitot-static probe. Temperature at the duct exit was not measured. All data were taken for a 60 second period at 1000 samples per second for each channel, beginning with ignition of the smoke cartridge.

During each experiment, the flow of smoke out of the duct was recorded on VHS video tapes using two cameras, for later analysis using the video imaging system. A shop fan was used to keep the smoke moving once it left the exit duct.

\section{Experimental Results}

Pressure Measurements at the Transducer Stand-off. At the beginning of the tests there was concern that a high temperature pressure transducer would be needed to measure the pressure buildup in the plenum chamber. However, after incorporating a stand-off in the plenum chamber pressure fitting, temperatures at the end of this six inch

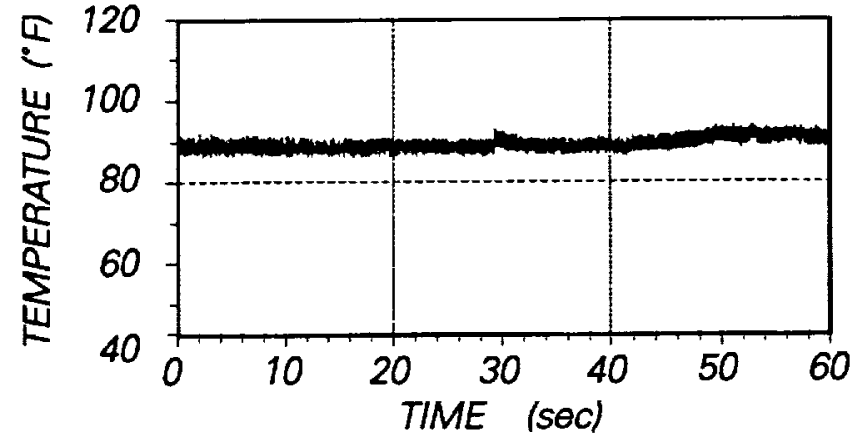

Figure 15. Temperature at Pressure Transducer Stand-off stand-off were measured to be sure that an available low temperature pressure transducer would not be damaged. The temperature measured at the end of the pressure transducer stand-off connected to the pressure transducer during test 1 is shown in Figure 15. Obviously, the stand-off prevents the temperature at the pressure transducer from rising above the recommended maximum temperature of $160^{\circ} \mathrm{F}$. Thus, use of the available transducer was justified.

Inadvertent Igniter Grounding. A grounding problem was encountered during initial tests. The problem occurred when the insulation on the ignitor wires burned off, allowing them to come into contact with the cartridge container or container cap. Because exposed junction thermocouples were used, electrical contact was made between the thermocouples and the ignitor wires. This contact caused errors in the voltages output by the thermocouple amplifiers. The problem was solved by disconnecting the power supply to the ignitor once the cartridge had begun to burn.

Valve Reliability. The first valve that was tested had a single solenoid and relied on a spring to return the spool when power was not applied to the solenoid. The residue that collected on the inside of the valve body and on the spools prevented the spring from shifting the spool reliably. The solenoid, however, always had sufficient power to shift the valve. A second valve, used in later tests, had two solenoids to shift the spool. This valve reliably shifted the spool, in spite of the residue buildup. The valves were disassembled and cleaned thoroughly after each test; and the valve seal and O-rings were inspected for tears. The seal on the second valve was pitted after four firings and was replaced. 


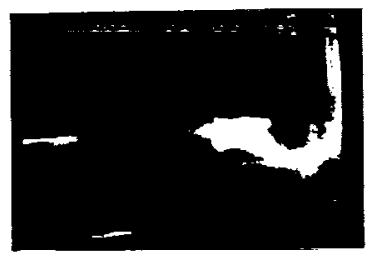

(a) $t=0.000 \mathrm{sec}$

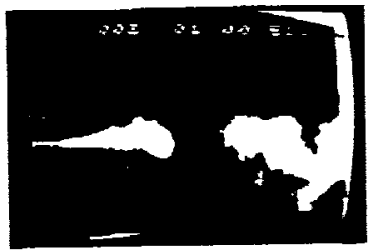

(c) $t=0.020 \mathrm{sec}$

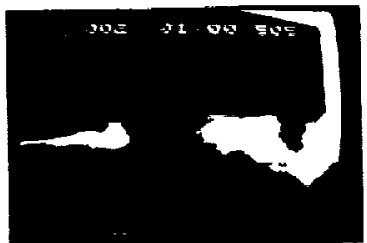

(b) $t=0.010 \mathrm{sec}$

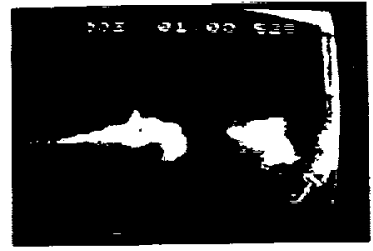

(d) $t=0.030 \mathrm{sec}$

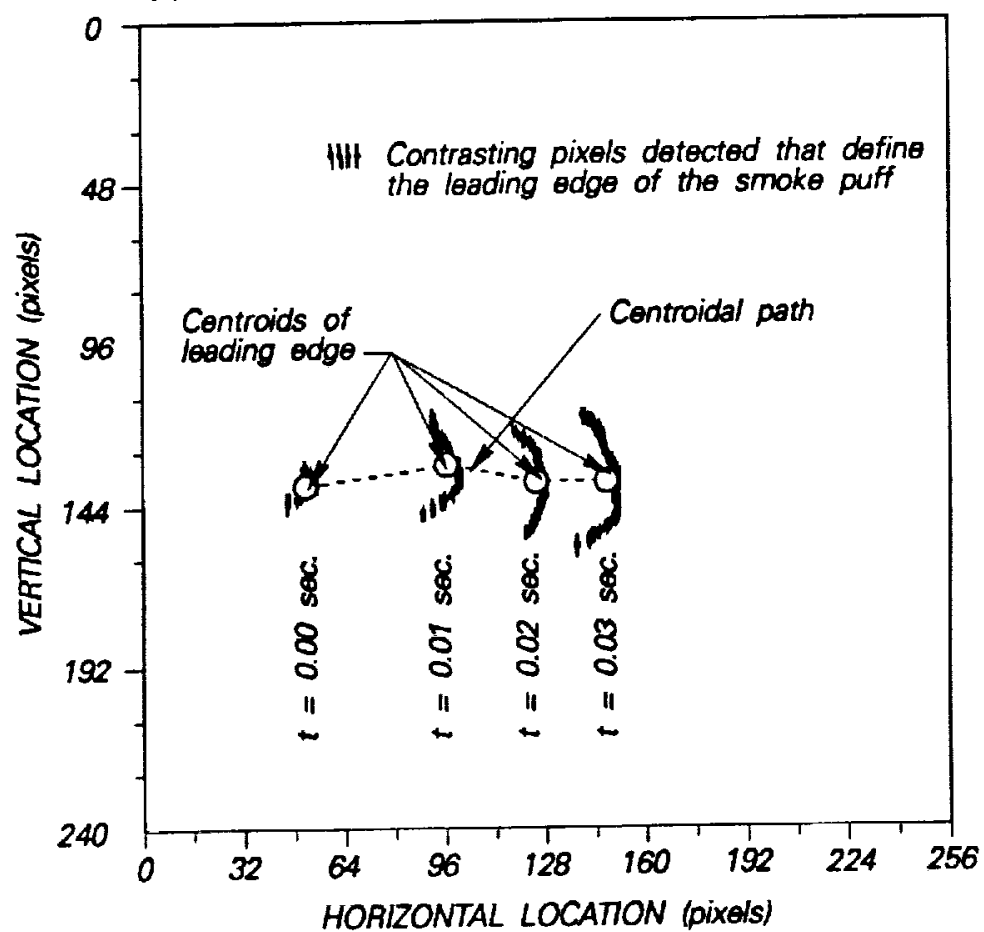

Figure 16. Camera 1 View of a Typical Smoke Puff Leading Edge

Coherence and Discreteness of Smoke Puffs. One of the major design requirements of the system is that it must produce discrete pulses of the visualization medium which can be identified on video tape. To demonstrate that the system meets this requirement the cartridge firings were filmed with two VHS cameras at a frame rate of 200 frames/sec. The video tapes were then analyzed using the video imaging system. Video data were digitized and the location of the line of contrast of the smoke puff leading edge was stored for each frame. The resulting files were then edited using the software's mask function to remove the lines of contrast which were not associated with the smoke puff's leading edge - for example, the contrast caused by the trailing end of the previous smoke puff. The leading edges of the smoke puff from the two camera views were then tracked using the trac function. This algorithm determines the centroid of the line of contrast and its location in a three-dimensional coordinate system for each 
frame. The resulting file gave the smoke puff's trajectory as a function of time. Each component was then differentiated numerically with respect to time to find the speed of the smoke puff in each coordinate direction.

Photographs of four frames from camera 1 of a typical smoke puff, as well as the resulting masked and centroid files, are shown in Figure 16. The frames shown were recorded 0.01 seconds apart. Figure 17 shows four frames of the same smoke puff as seen by camera 2. Because of a limitation of the VCR used when photographing the frames they do not correspond to those shown in Figure 16. Instead they lag behind by 0.005 seconds. It is clear that the leading edge of the smoke puff was recorded faithfully and digitized by the automated video imaging hardware and software. The location of the smoke puff downstream of the exit duct is shown in Figure 18 and its speed in the corresponding direction is shown in Figure 19.

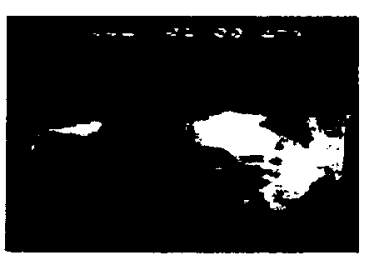

(a) $\mathrm{t}=0.005 \mathrm{sec}$

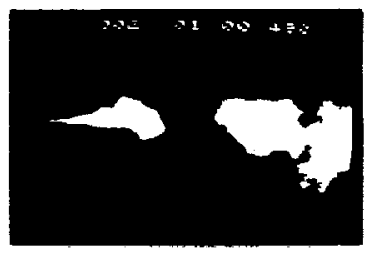

(c) $t=0.025 \mathrm{sec}$

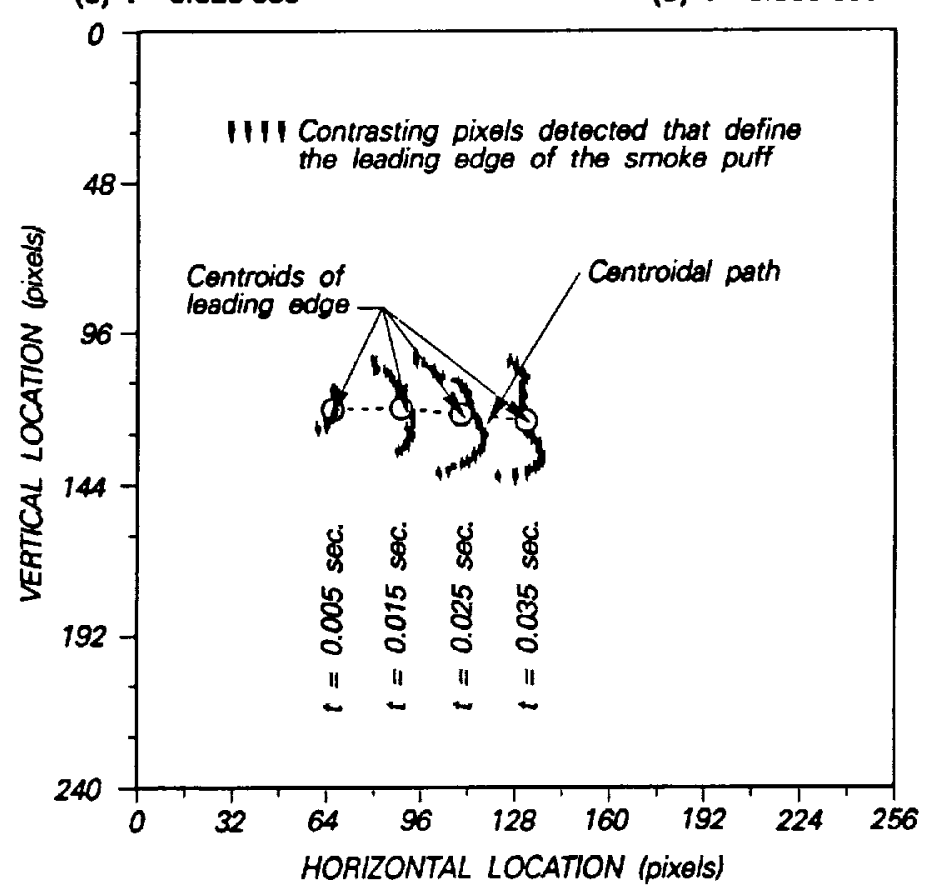

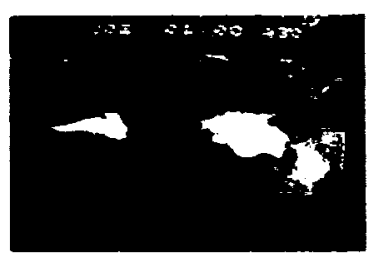

(b) $t=0.015 \mathrm{sec}$

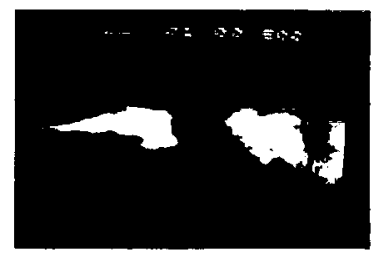

(d) $\mathrm{t}=\mathbf{0 . 0 3 5 \mathrm { sec }}$
LOCATION Ipikas

Figure 17. Camera 2 View of a Typical Smoke Puff Leading Edge 


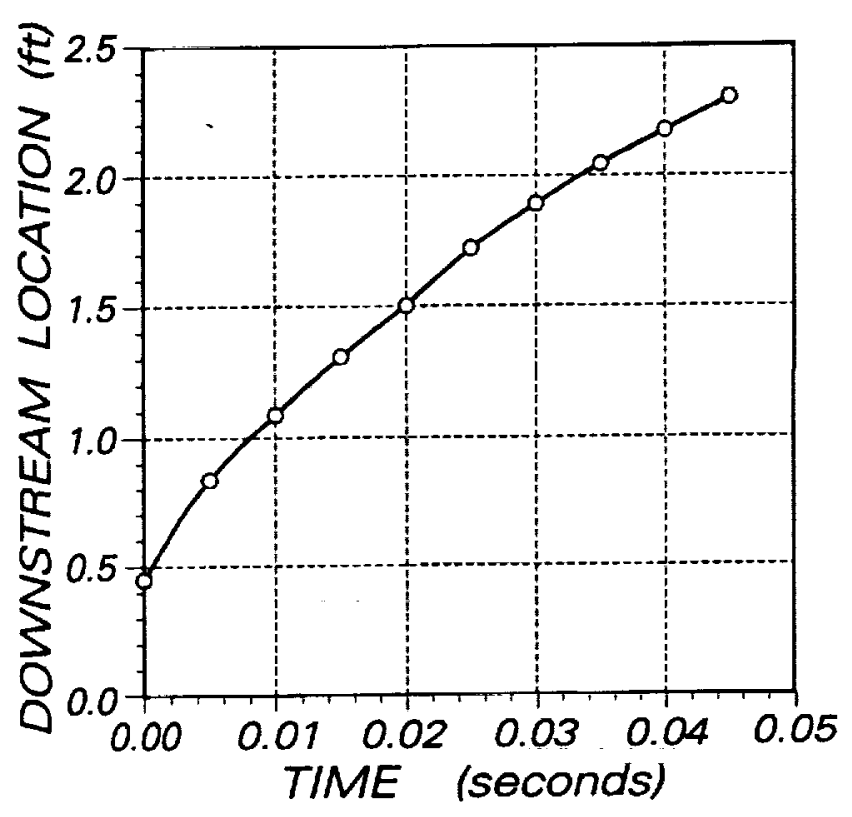

Figure 18. Downstream Location of Smoke Puff Leading Edge

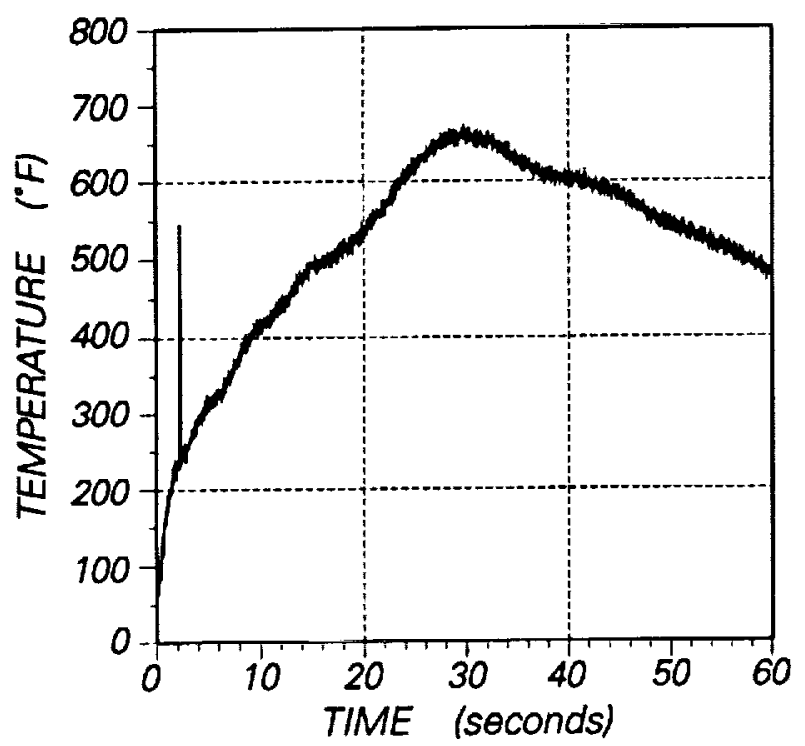

Figure 20. Temperature of Gaseous Mixture Exiting the Smoke Cartridge

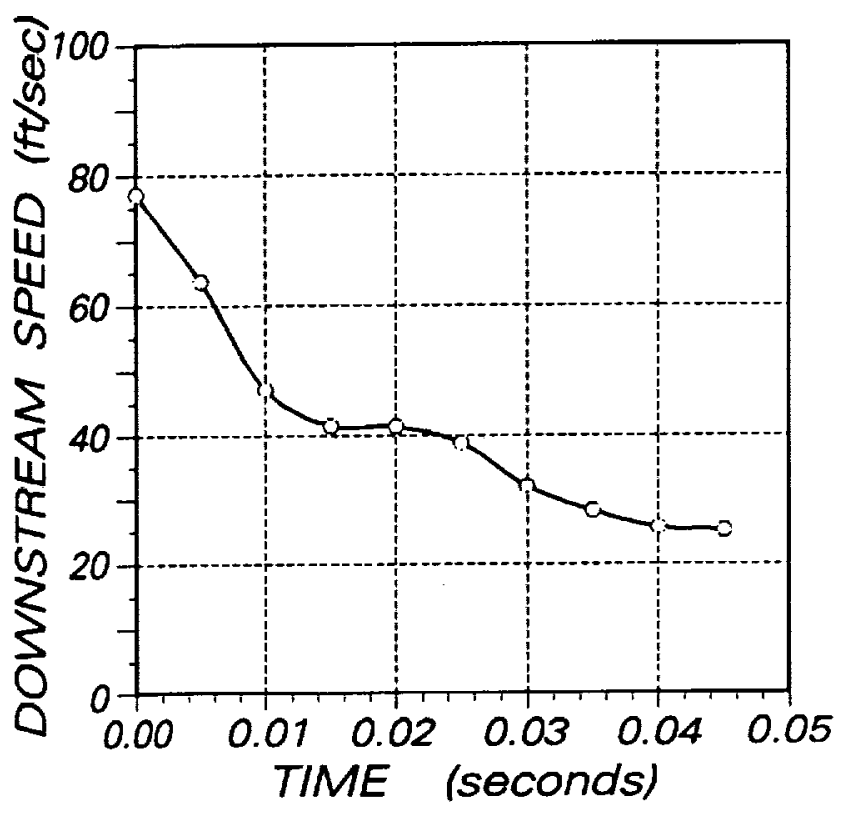

Figure 19. Downstream Speed of Smoke Puff Leading Edge

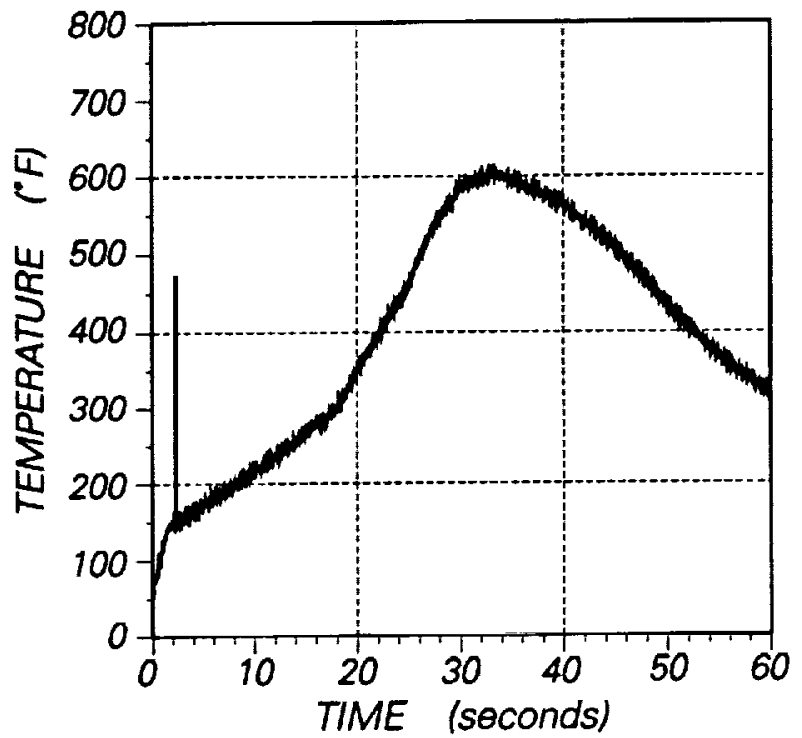

Figure 21. Temperature of Gaseous Mixture in the Plenum Chamber

System Temperatures. Temperature measurements were recorded for three locations in the system. The temperature of the smoke exiting the pyrotechnic cartridge was successfully measured four times. The maximum of this measurement ranged from $660^{\circ} \mathrm{F}$ to $700^{\circ} \mathrm{F}$, with an average of $680^{\circ} \mathrm{F}$. The temperature in the plenum chamber was successfully measured five times, with a maximum temperature ranging from $560^{\circ} \mathrm{F}$ to $640^{\circ} \mathrm{F}$, and an average of $600^{\circ} \mathrm{F}$. The temperature of the smoke at the duct exit was measured twice. The maximum temperatures were $560^{\circ} \mathrm{F}$ and $610^{\circ} \mathrm{F}$, 
for an average of $585^{\circ} \mathrm{F}$. Data for the three temperature measurements taken during the firing of cartridge 8 are shown in Figures 20, 21, and 22.

System Pressure. Five tests were conducted in which the differential between the pressure inside the plenum chamber and ambient pressure was successfully measured. During the firing of smoke cartridge 5 , the flow of smoke was repeatedly switched between two valve ports. During tests 8,9 and 10 the valve was used to repeatedly block the flow of smoke; three different plenum volumes were tested to determine the effect of system volume on the rise in pressure while the flow of smoke was blocked.

Pressure data from test 5 are shown in Figure 23. The large pressure spike typically occurred as the smoke cartridge purged itself of carbon ash. This purging happened during each firing, although the amplitude of the pressure spike was different from cartridge to cartridge. The carbon ash caused the smoke to briefly turn black, but it quickly returned to its normal white color. Excluding the pressure spike, the higher pressures correspond to segments of time when denser smoke was produced by the system. The maximum differential pressure in the plenum chamber during the production of usable smoke with the valve merely diverting the smoke was 4.5 psid. Clearly, diverting the smoke between two different exit ports caused no significant pressure rises.

In tests 8,9 , and 10 two different valve

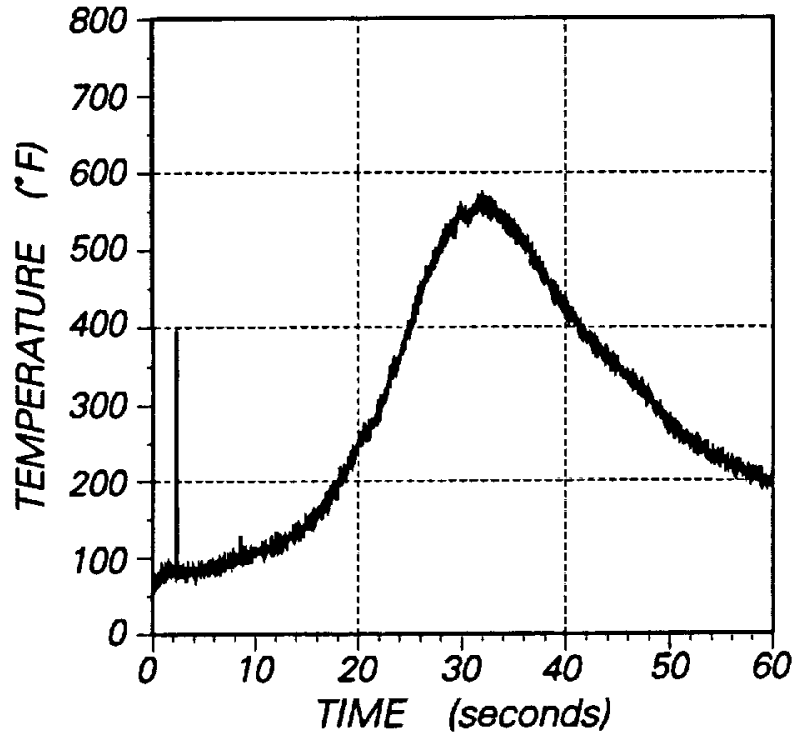

Figure 22. Temperature of Smoke at the Duct Exit

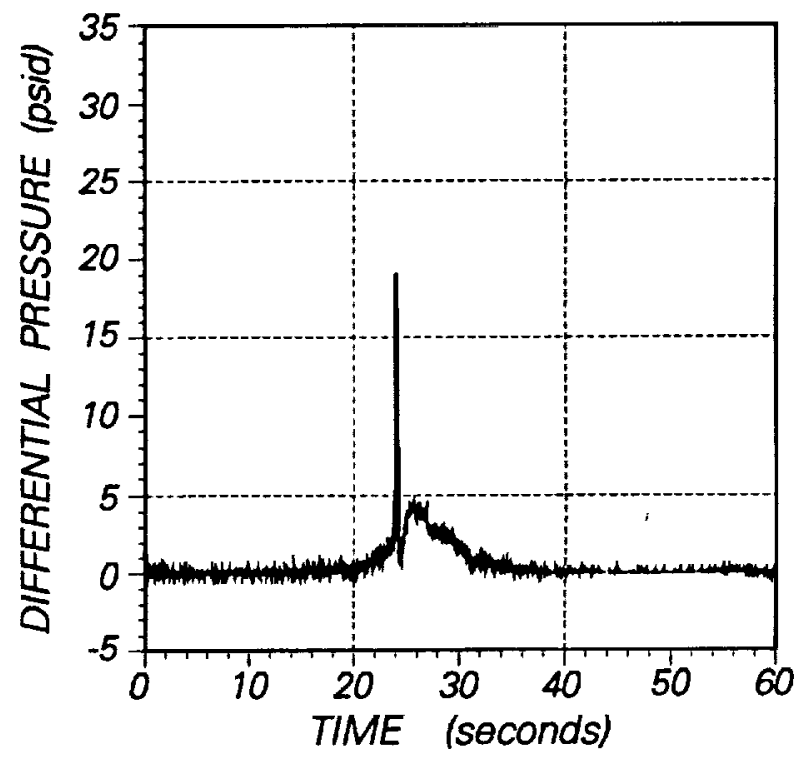

Figure 23. Plenum Chamber Pressure Differential with Smoke Flow Diverted cycling frequencies were used as the flow of smoke was repeatedly blocked. Since the volume of the system was increased with each test, the time the valve could remain closed increased. The longer close times gave a longer gap between smoke pulses. However, longer close times also required the valve to remain open longer between closings to allow the pressure to return to its undisturbed value. This delay reduced the number of usable pulses of smoke obtained from each cartridge. Valve sequencing parameters are given in Table 12. Valve close time is actually the time that the relay controlling the valve was signaled to switch the valve to the closed position. This time 
increment includes the response time of the relay and the valve. The response time of the relay is uncertain and varies from cycle to cycle up to 8.3 msecs. This uncertainty is due to its dependence on the phase of the line power when the relay is signaled to switch the valve. Valve cycle time is the time between signals to close the valve. The number of cycles is the number of cycles the valve switched at that frequency before alternating with the other frequency.

Table 12. Timing of Valve Sequences

\begin{tabular}{|c|c|c|c|}
\hline Test Number & $\begin{array}{c}\text { Valve Close Time } \\
\text { (msecs) }\end{array}$ & $\begin{array}{c}\text { Valve Cycle Time } \\
\text { (msecs) }\end{array}$ & Number of Cycles \\
\hline 8 & 38 & 150 & 12 \\
\hline 8 & 58 & 200 & 11 \\
\hline 9 & 40 & 250 & 6 \\
\hline 9 & 70 & 300 & 5 \\
\hline 10 & 55 & 300 & 5 \\
\hline 10 & 90 & 350 & 6 \\
\hline
\end{tabular}

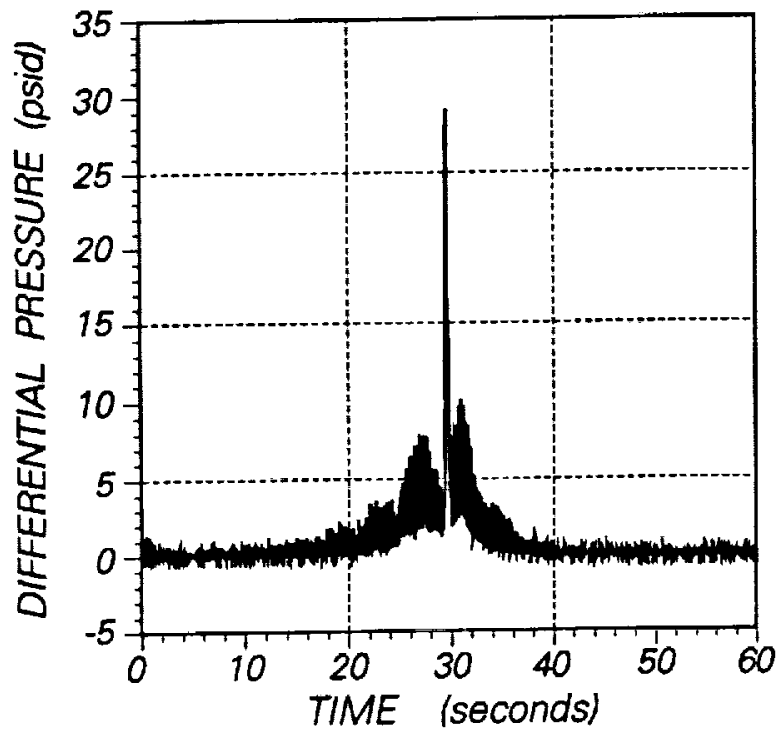

Figure 24. Plenum Chamber Pressure During Test Number 8

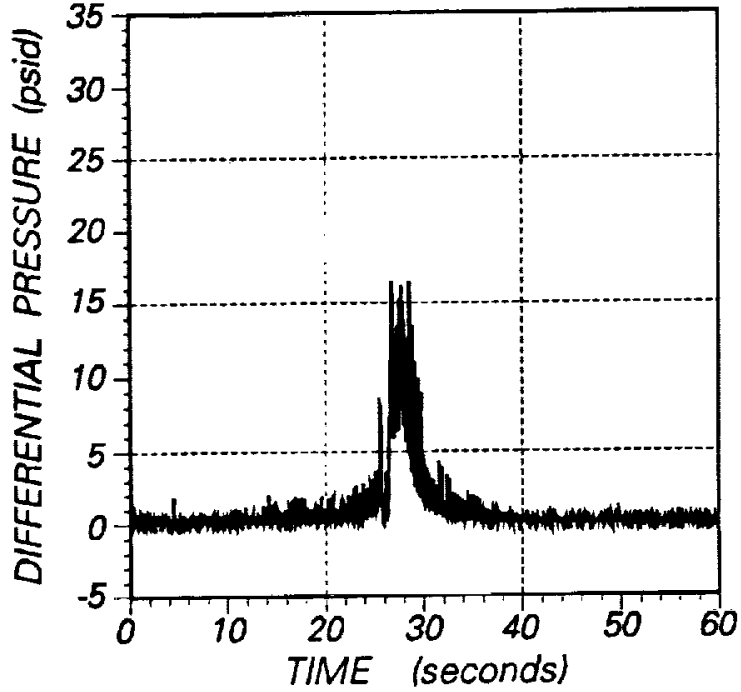

Figure 25. Plenum Chamber Pressure During Test Number 9

Figures 24, 25, and 26 show the pressure data from tests 8,9 , and 10, respectively. It is evident that blocking the flow of smoke caused only small pressure rises in the system. It did not raise the pressure to anywhere near the rupture disk burst pressure of 55 to 75 psid. In fact the worst case pressure rise in test number 8 still left a margin of approximately half of the design rupture disk burst pressure.

The pressure changes caused by six valve openings during test 9 are shown in more detail in Figure 27. The pressure begins to rise as the valve closes and then, when the valve reopens, the pressure decays exponentially until the valve closes again. 
Figures 23-26 show that the cartridge burn rate varied from test to test. The maximum pressure which occurred at the beginning of a valve closing, or the "maximum undisturbed" pressure, is a critical factor, since it corresponds to maximum burn rate, and thus maximum mass flow rate into the system. The maximum undisturbed pressure for each test is given in Table 13. There was considerable variance in this maximum undisturbed pressure from test to test. One possible reason is that the increased pressure that results from blocking the smoke flow may increase in the cartridge burn rate. The extent of this increase for

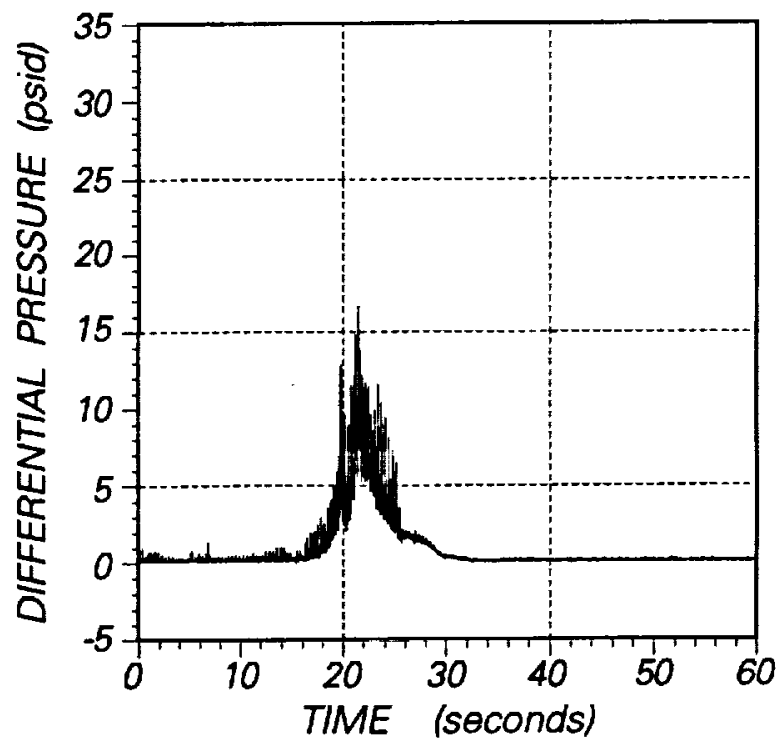

Figure 26. Plenum Chamber Pressure During Test Number 10 the pressures encountered during testing is not known. Evidence that the increased pressure did not alter the burn rate is that the lowest maximum undisturbed pressure occurred during test number 8 in which the method of pulsing the smoke was blocking the flow. Because the number of cartridges available for testing was limited, no attempt was made to investigate the cause of the variation in cartridge burn rate.

Table 13. Maximum Undisturbed Plenum Chamber Differential Pressure

\begin{tabular}{||c|c|c|}
\hline Test & $\begin{array}{c}\text { Maximum } \\
\text { Undisturbed } \\
\text { Pressure } \\
\text { (psid) }\end{array}$ & $\begin{array}{c}\text { Method of } \\
\text { Pulsing } \\
\text { Smoke }\end{array}$ \\
\hline 5 & 4.5 & Diverting \\
\hline 6 & 4.6 & Blocking \\
\hline 8 & 2.7 & Blocking \\
\hline 9 & 8.0 & Blocking \\
\hline 10 & 5.4 & Blocking \\
\hline
\end{tabular}

Smoke Exit Velocity. During tests 9 and 10 the dynamic pressure of the smoke at the

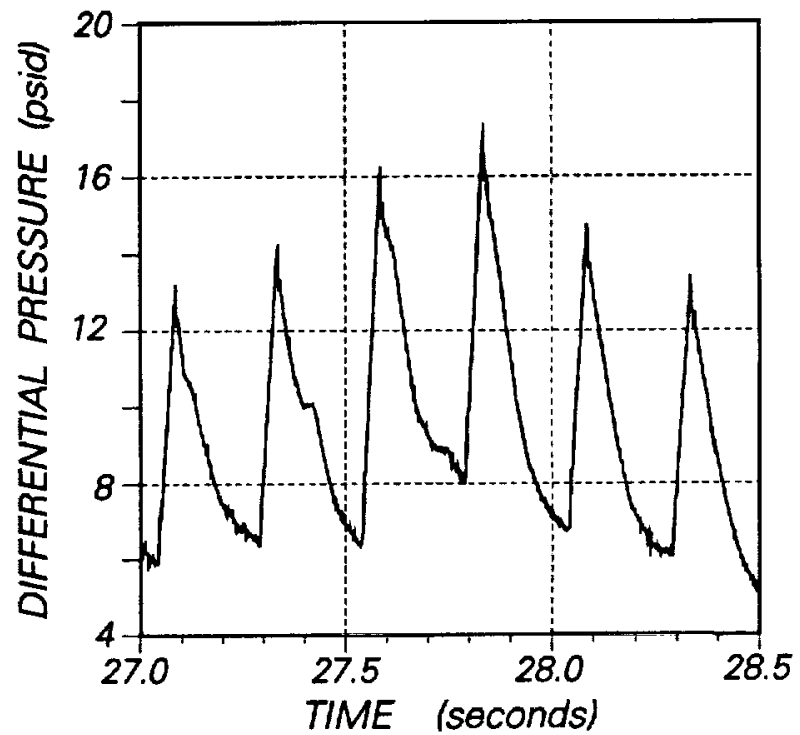

Figure 27. Plenum Chamber Differential Pressure During Six Valve Cycles of Test Number 9 duct exit was measured using a pitot-static probe located in the center of the tube cross-section approximately 0.7 inches from the exit plane. This dynamic pressure, along with the temperature of the smoke (determined by averaging the maximum exit temperatures from the previous tests), and 
the ambient pressure was used to determine the velocity of the smoke at the duct exit. The velocities from these two tests are shown in Figures 28 and 29.

The apparent negative velocities are the result of vibration of the pressure transducer diaphragm due to the sudden drop in dynamic pressure when the flow of smoke stopped. Figure 30 shows the velocity of a single smoke puff during test 10 in which the vibration of the diaphragm is readily apparent. Figure 31 shows the velocity of the smoke puffs during test number 9 for which the pressure data were presented in Figure 28. When the valve opened, the higher pressure in the system caused the smoke to exit with a greater velocity. As the pressure decayed the velocity of the smoke exiting the duct decreased also.

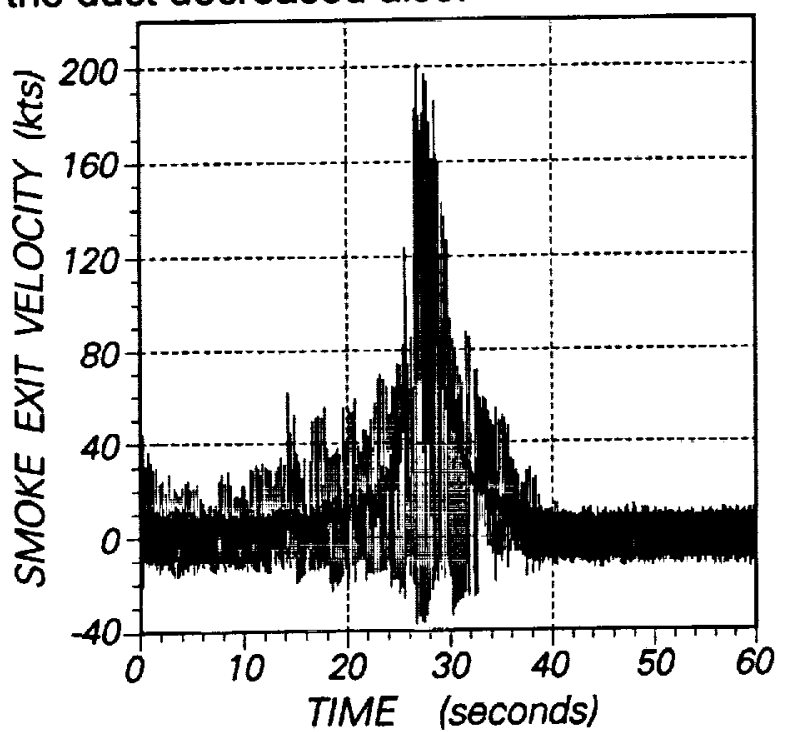

Figure 28. Measured Smoke Exit Velocity During Test Number 9

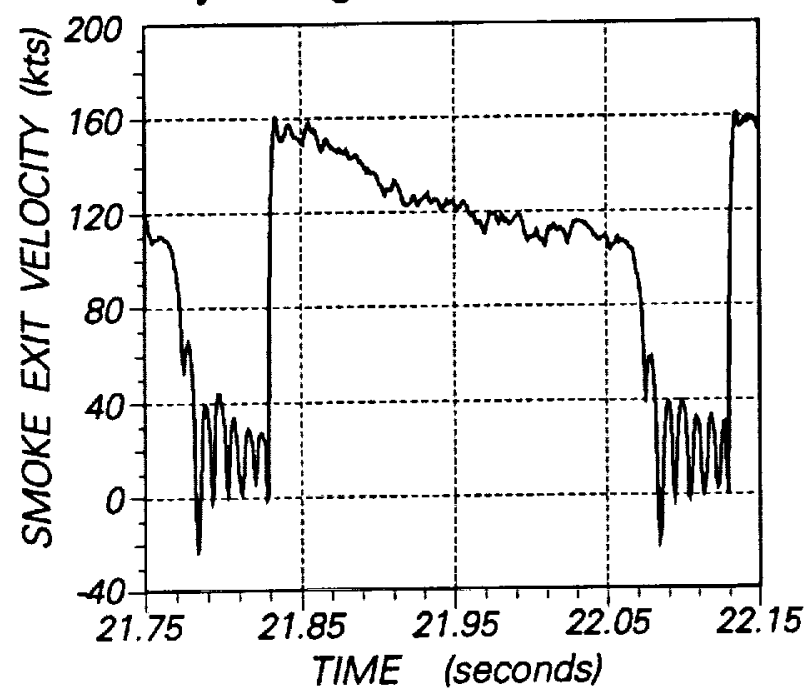

Figure 30. Measured Smoke Exit Velocity During Test Number 10

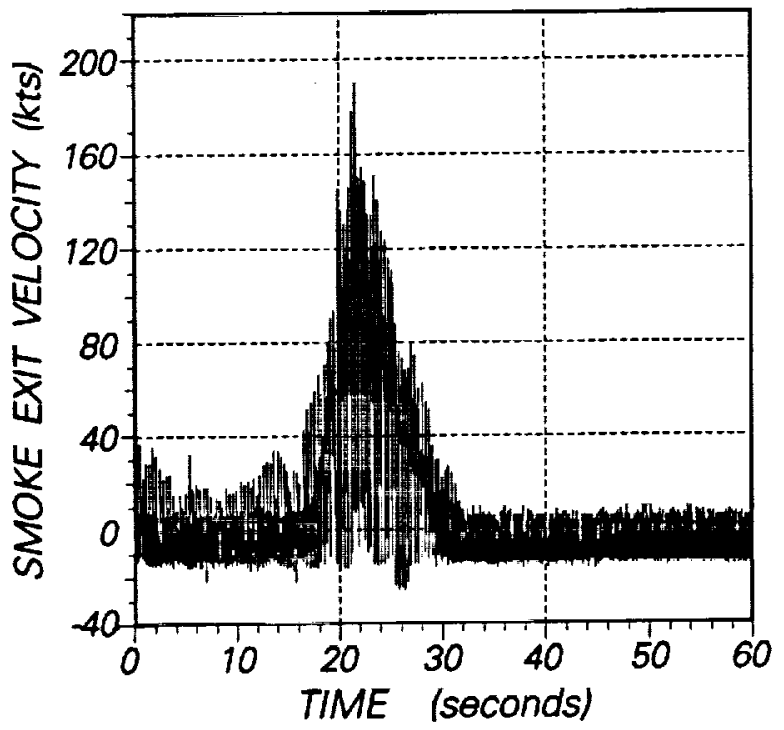

Figure 29. Measured Smoke Exit Veloc-

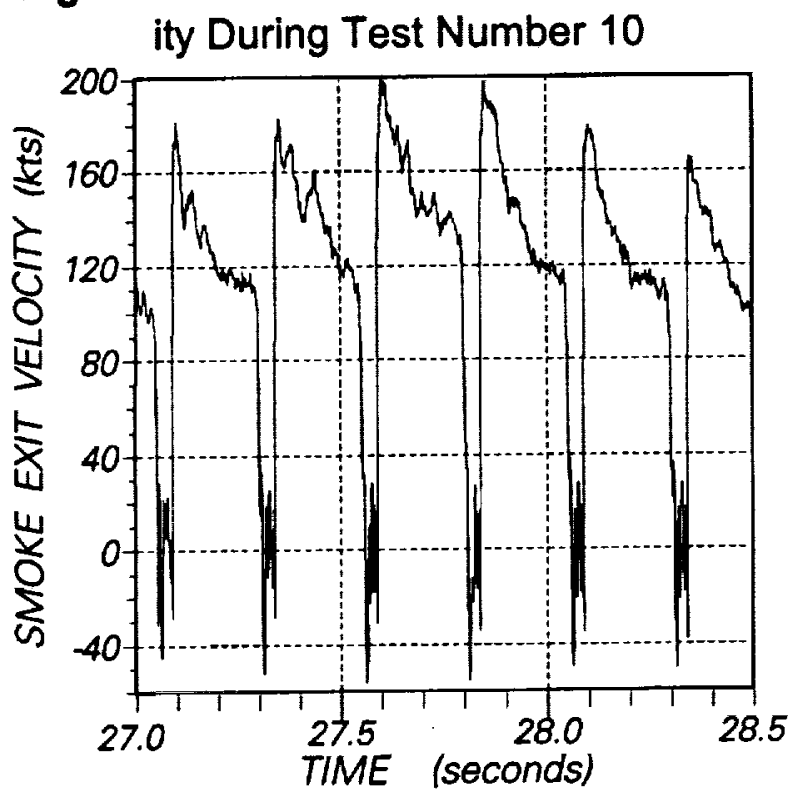

Figure 31. Measured Smoke Exit Velocity During Test Number 9 


\section{Comparison of Predicted and Measured Pressures and Velocities}

The conservation of mass and energy equations discussed previously were integrated numerically for several initial conditions and system parameter combinations, and the results were compared to the pressure and velocity measurements from tests 8 through 10. Initial conditions needed for the integration included: initial temperatures of the smoke entering the system and in the plenum chamber, ambient pressure, and initial plenum chamber differential pressure. Two system parameters were also varied, the valve sequence timing and system volume. The estimated temperature of the smoke entering the system for all cases was $680^{\circ} \mathrm{F}$, the initial system temperature was estimated to be $600^{\circ} \mathrm{F}$. The valve total flow capacity used for all simulations was assumed to be $5.1,10 \%$ less than the factory listed values of 5.7 given by Benedict 27 .

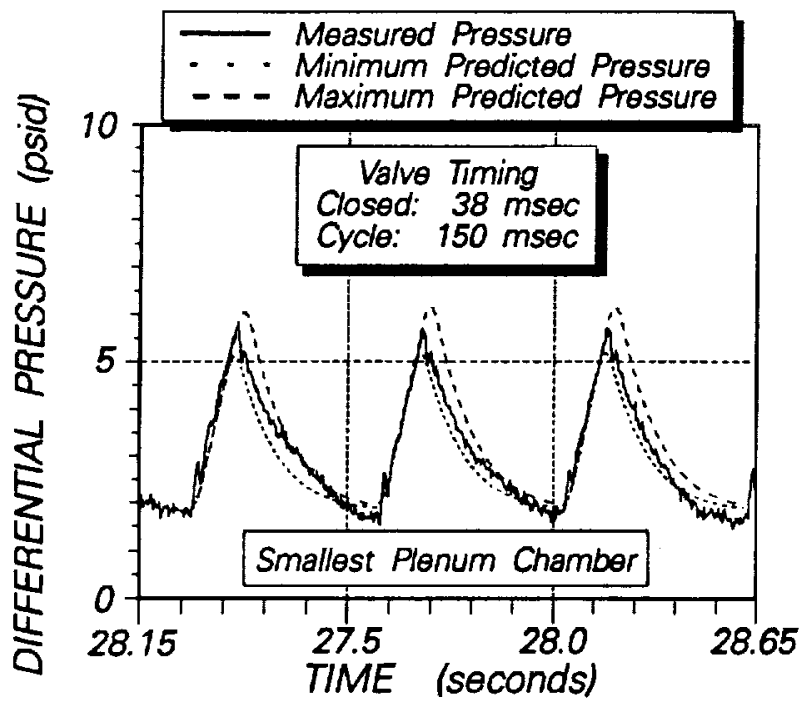

Figure 32. Comparison of Measured and

Predicted Plenum Chamber Pressure During Test Number 8

To compare the predicted pressures

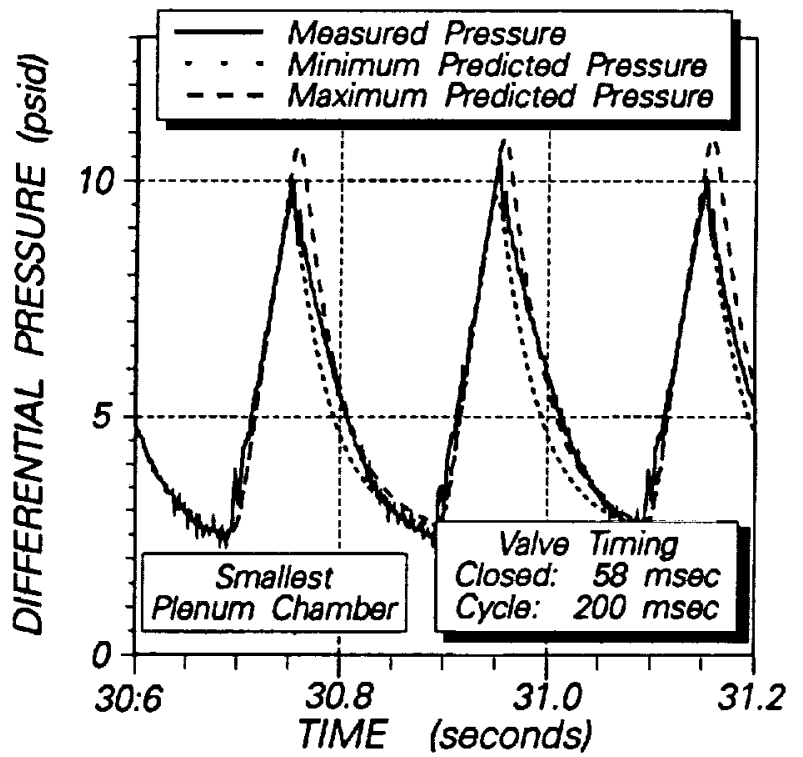

Figure 33. Comparison of Measured and Predicted Plenum Chamber Pressure During Test Number 8

and the measured pressures, two valve sequences were run for each set of initial conditions. This approach was taken because of the uncertainty in the response time of the relay controlling the valve. The two predictions gave minimum and maximum pressure and velocity estimates, between which measured data should lie. Six comparisons of measured and predicted pressures and velocities and are shown in Figures 32 through 41 . The system parameters and initial conditions are given in Table 14. As can be seen in the figures, the predicted pressures and velocities match the measured values well for all the valve sequences, system volumes, and initial differential pressures.

It is important to note that diverting the flow of smoke between two exit ports is the trivial case of no pressure build-up. As the flow capacity of one port decreases, the flow capacity of the other increases, so that the total flow capacity remains constant. 
But, as previously noted, even when the valve is completely blocked off for short periods of time, the pressure buildup is still within the rupture pressure limits for the system.

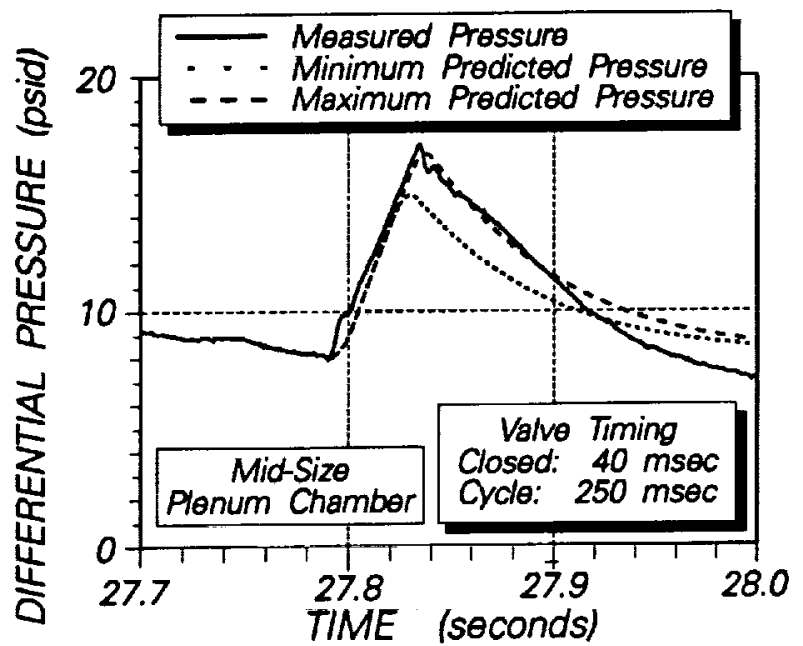

Figure 34. Comparison of Measured and Predicted Plenum Chamber Pressure During Test Number 9

Table 14. System Parameters for Comparison of Pressures and Velocities in Test Numbers 8 through 10

\begin{tabular}{|c|c|c|}
\hline $\begin{array}{c}\text { Test } \\
\text { Number }\end{array}$ & $\begin{array}{c}\text { System Vol- } \\
\text { ume }\left(\mathrm{in}^{3}\right)\end{array}$ & $\begin{array}{c}\text { Ambient Pres- } \\
\text { sure }(\mathrm{psi})\end{array}$ \\
\hline 8 & 100.7 & 14.88 \\
\hline 9 & 125.7 & 14.65 \\
\hline 10 & 175.7 & 14.72 \\
\hline
\end{tabular}

As shown in Figures 36 and 37, velocity Measurements for Test Number 9 also showed rather good correlation with the predicted velocity at the exit tube orifice. As with the pressures, there were two predicted values based on the bounds of the relay opening times. There are two points worth emphasizing about these comparisons: (1) the velocity measurements were made with

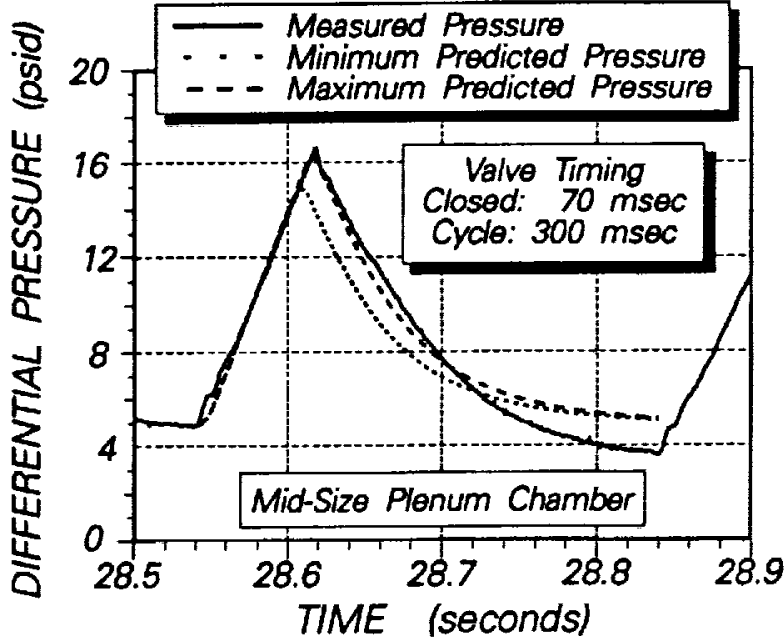

Figure 35. Comparison of Measured and Predicted Plenum Chamber Pressure During Test Number 9

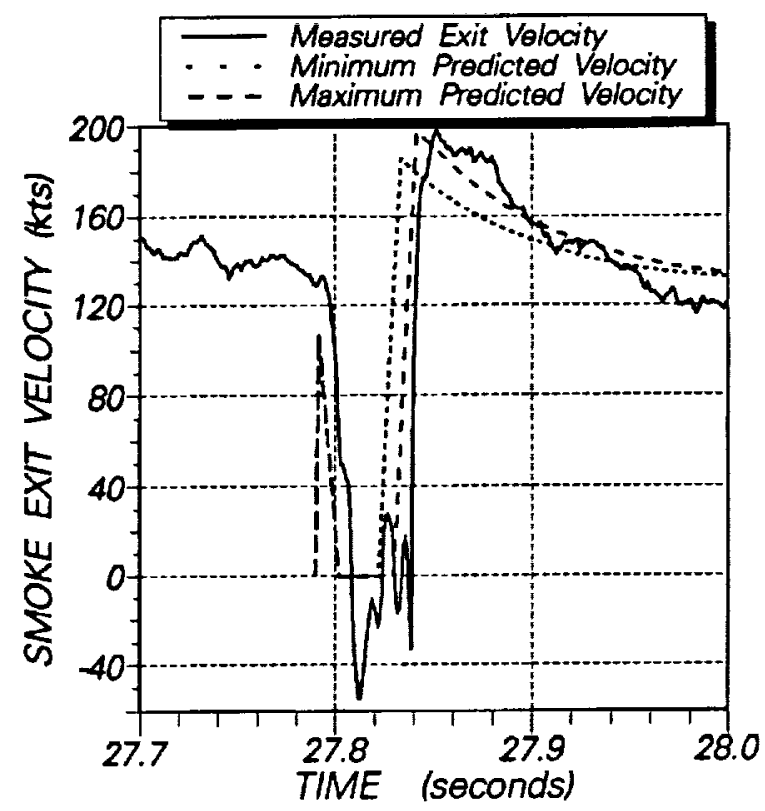

Figure 36. Measured and Predicted Smoke Exit Velocities (Test 9) a simple pitot tube and no attempt was made to correct for restricted tube area as the smoke residue built up on the pitot tube and (2) the transducer "ringing" (noted earlier) does not give a true steady state signal. However, the average value during these resonances of the transducer diaphragm appears to be representative of the actual dynamic pressure at the duct exit. 
For the mid-sized plenum chamber of Table 14, Figures 38 and 39 show rather good correlation between the predicted smoke velocity at the exit tube orifice. As with the plenum chamber pressures there were two predicted values of exit velocity, based

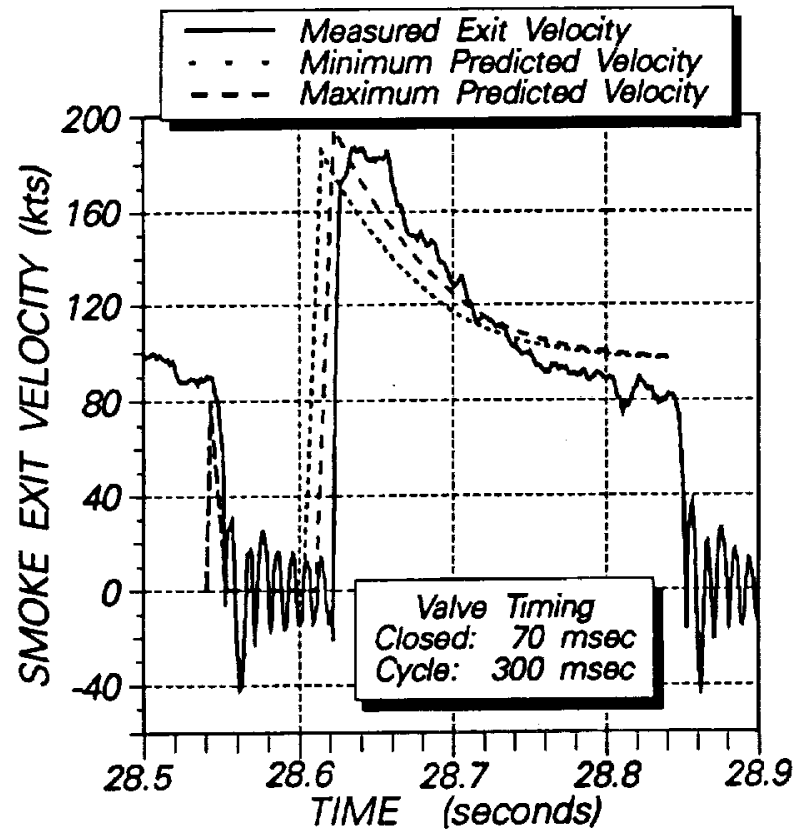

Figure 37. Measured and Predicted

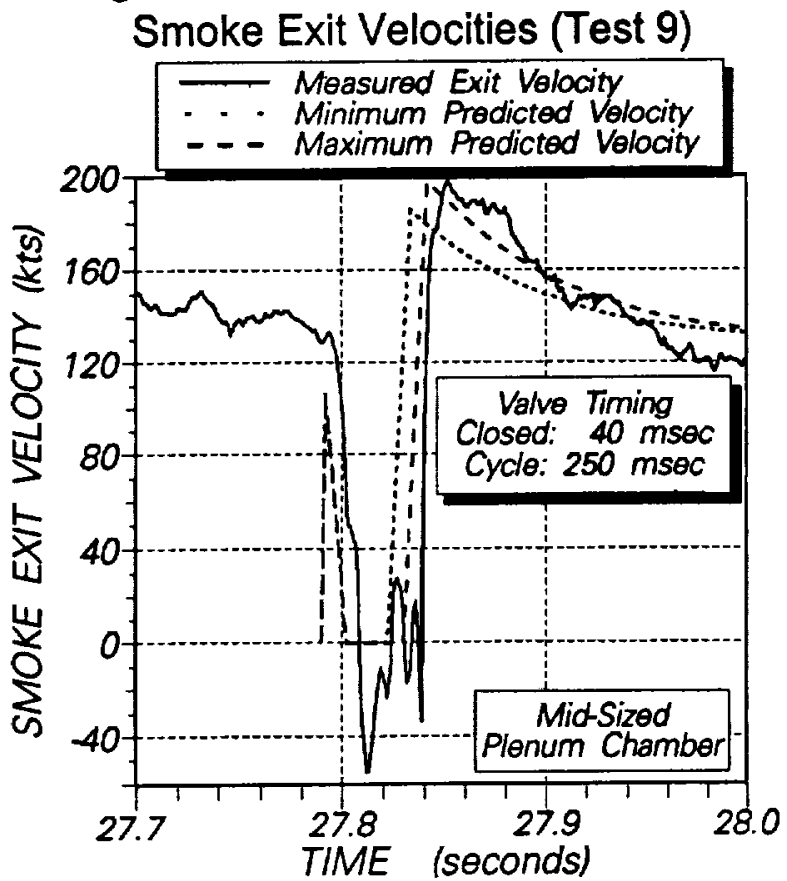

Figure 38. Measured and Predicted Plenum Chamber Pressures (Test 10) on the uncertain bounds of the relay opening times. Figures 40 and 41 , for the largest plenum chamber tested, show similarly good predictions. Of course, the maximum velocities at the duct exit are about 20 to 30 knots lower than for the mid-sized plenumb chamber. This difference evidently occurred because the corresponding driving pressures were lower by about 5 to 7 psi (Figures 34-38). The pressure and the exit velocity were lowered even though the valve closed times increased from $\mathbf{4 0}$ to $\mathbf{5 5}$ milliseconds and from 70 to 90 milliseconds. Clearly, larger plenum chamber volumes were quite effective in providing additonal safety margins.

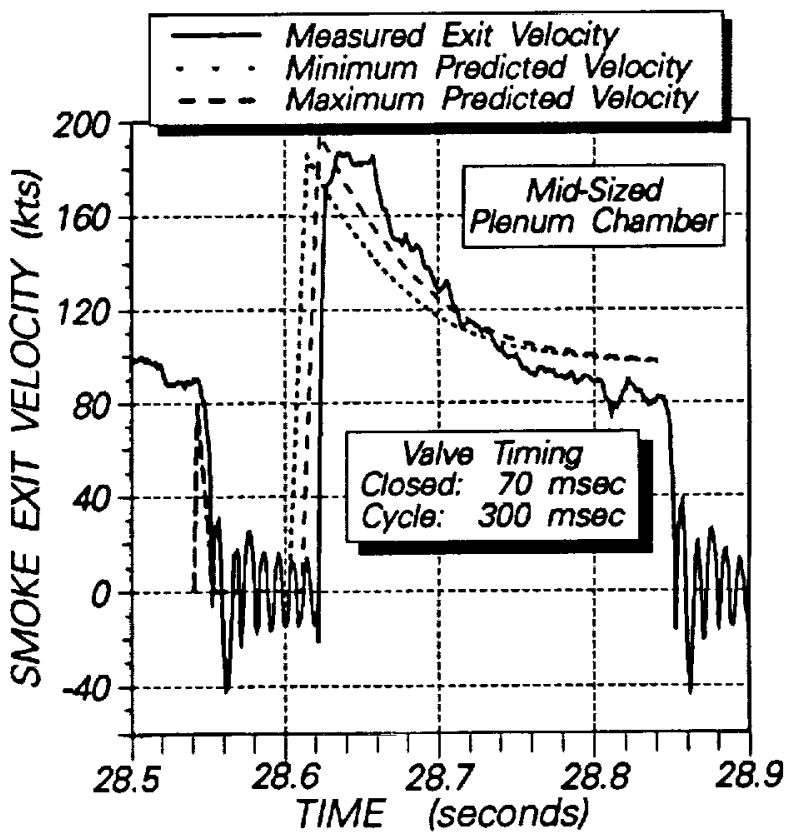

Figure 39. Measured and Predicted Plenum Chamber Pressures (Test 10)

Pressure and Exit Velocity Modelling

Given an initial plenum chamber differential pressure, the thermodynamic model of the system successfully predicts the pressure buildup when the flow of smoke is blocked, as well as the initial exit velocity of 
the smoke after the valve is opened. The model is valid for a variety of system volumes and valve closing sequences and may be used to predict the pressure and exit velocities for system parameters which have not yet been tested.

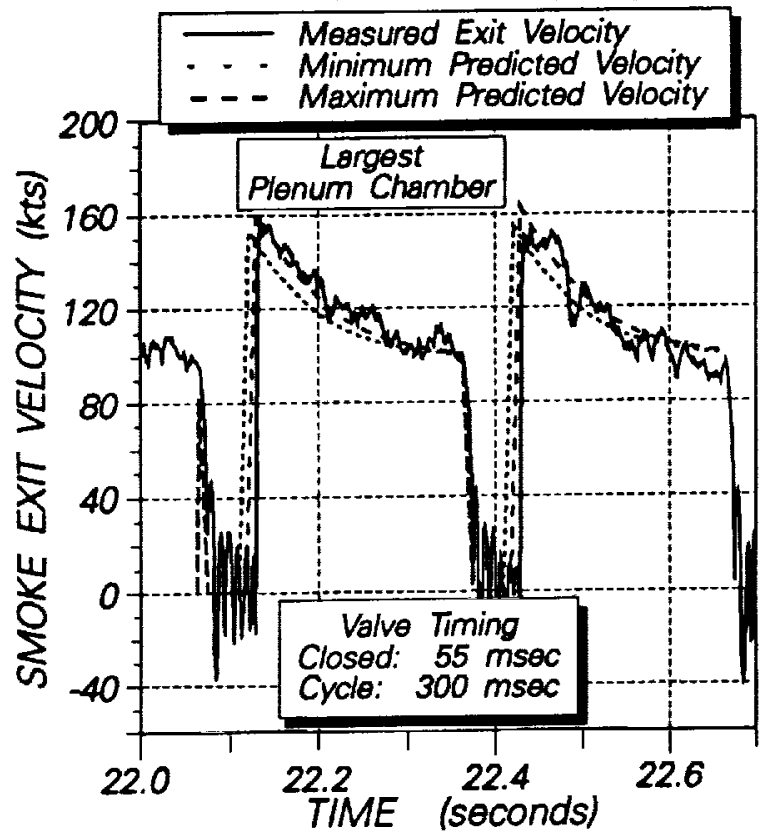

Figure 40. Measured and Predicted

Smoke Exit Velocities (Test 10)

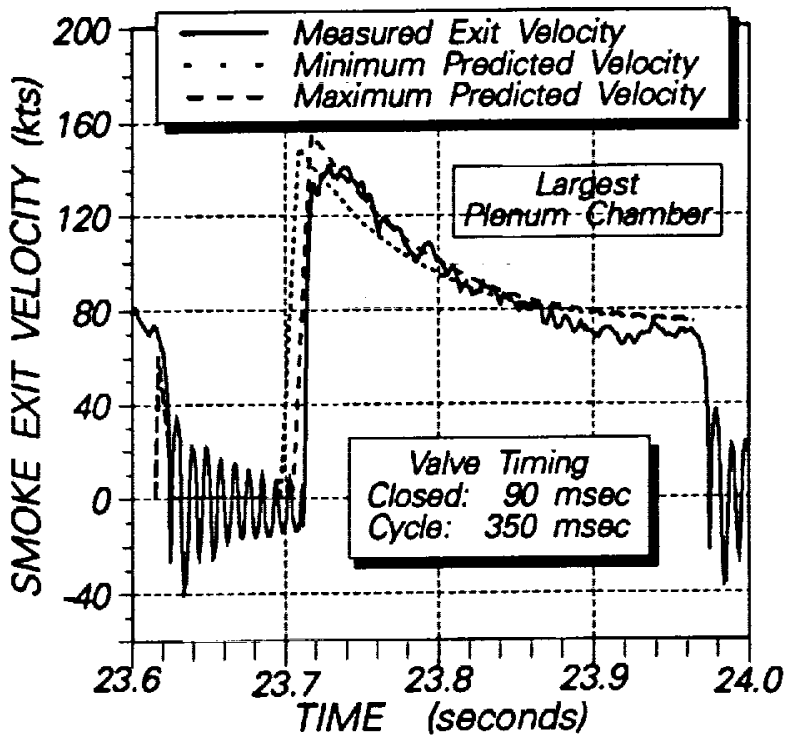

Figure 41. Measured and Predicted Smoke Exit Velocities (Test 10)

The model does have limitations, of course. First, there is uncertainty as to what value of maximum undisturbed pressure should be used as the initial condition. For the five tests conducted maximum undisturbed pressures ranged from 2.7 psid to 8.0 psid. The mean was 5.0 psid, and the standard deviation was 1.7 psid. The sparsity of data makes it difficult to determine what pressure should be used as an initial condition for the model. However, as more data are collected in future tests, this problem may be alleviated.

One important case to consider is that of a system with multiple cartridges burning at the same time. This approach will increase the mass flow rate into the system and, hence, the maximum undisturbed pressure. The maximum mass flow rate into the system is expected to increase in multiples of that encountered when firing one cartridge according to the number of cartridges fired. That is, firing two cartridges simultaneously should double the maximum undisturbed pressure. The mass flow rate through the valve is shown in Figure 42 as a function

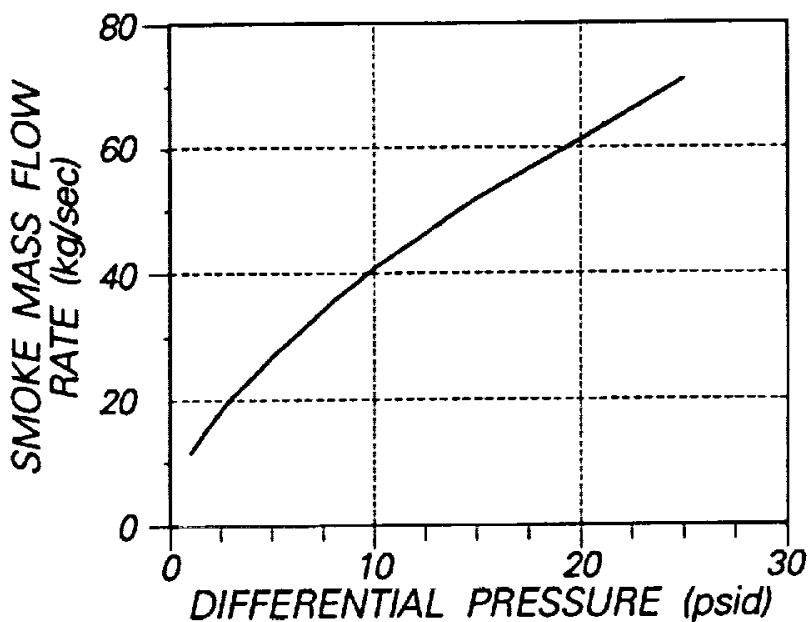

Figure 42. Mass Flow Rate of Smoke through the Open Valve of plenum chamber differential pressure.

To determine the differential pressure to be used as a pressure model initial condition 
for multiple cartridges, first select the maximum undisturbed differential pressure for one cartridge and determine the mass flow rate for that pressure. Multiply the single cartridge mass flow rate by the number of cartridges fired simultaneously and locate the plenum chamber differential pressure corresponding to that mass flow rate.

Another limitation of the model concerns valves with different flow capacity, or tubing with a smaller inside diameters. The maximum undisturbed pressure data collected to date are not likely to apply because of the effect of pressure on the cartridge burn rate. A valve with a lower flow capacity, or smaller tubing, will increase the pressure in the system, which may in turn increase the burn rate. A valve with a larger flow capacity will decrease the pressure and the cartridge burn rate will also likely decrease.

\section{Effect of Smoke on the Flow Field}

One important issue whenever a seeding medium is used for flow visualization is its effect on the flow field. The two important factors when determining the extent of the effect of the seeding medium are its velocity and mass flow rate as it enters the flow field. Extensive research has been conducted to determine the effect of blowing vortices on aircraft forebodies and swept leading edges, including strakes, especially at high AOAs. The purpose of these studies was to determine the feasibility and effectiveness of improving control of the aircraft by controlling the vortical flow by injecting air into the flow field near the origin of the vortex. In each study an optimum blowing port location and orientation was determined to maximize the effectiveness of the blowing for the configuration being considered. A blowing momentum coefficient defined as:

$$
C_{\mu} \equiv \frac{\dot{m} V}{q_{\infty} S}
$$

where $m$ is the mass flow rate of the visualization medium and $V$ is the velocity as it enters the flow field, was calculated to provide a nondimensional method for comparing data. Although the visualization medium differs from the air that was used in that it contains solid particles, and the smoke exit port is not in the optimal location or orientation for affecting the vortex core, it is appropriate to examine the blowing momentum coefficient of the smoke to gain some insight into its effect on the flow field.

Bradley and Wray 28 have shown in wind tunnel tests that for aircraft with a strake configuration, blowing along the strake can strengthen the vortex core and delay vortex bursting. This is quantifiable in terms of an increased $C_{L}$. For a the case of blowing the strake vortex only, on a generic strake configuration with a $C_{\mu}$ of $0.025, C_{L_{\max }}$ increased approximately $10 \%$; a $C_{\mu}$ of 0.084 caused a $C_{L_{\max }}$ increase of approximately $33 \%$. A wind tunnel study by Skow, et al, 29 has shown that a much lower blowing coefficient is needed to alter forebody vortices on an F-5F. A $C_{\mu}$ of only 0.008 was sufficient to change the positions of the vortices to a mirror image reflection of the unblown case at AOAs above $30^{\circ}$, with $\beta=0^{\circ}$. This blowing momentum coefficient caused a reversal in the direction of the yawing moment. However, the minimum $C_{\mu}$ needed to cause the vortex position change was not determined. This critical blowing momentum coefficient needs to be addressed systematically, both analytically and experimentally, if forebody vortex control is to be exploited as a component of high AOA control systems. 


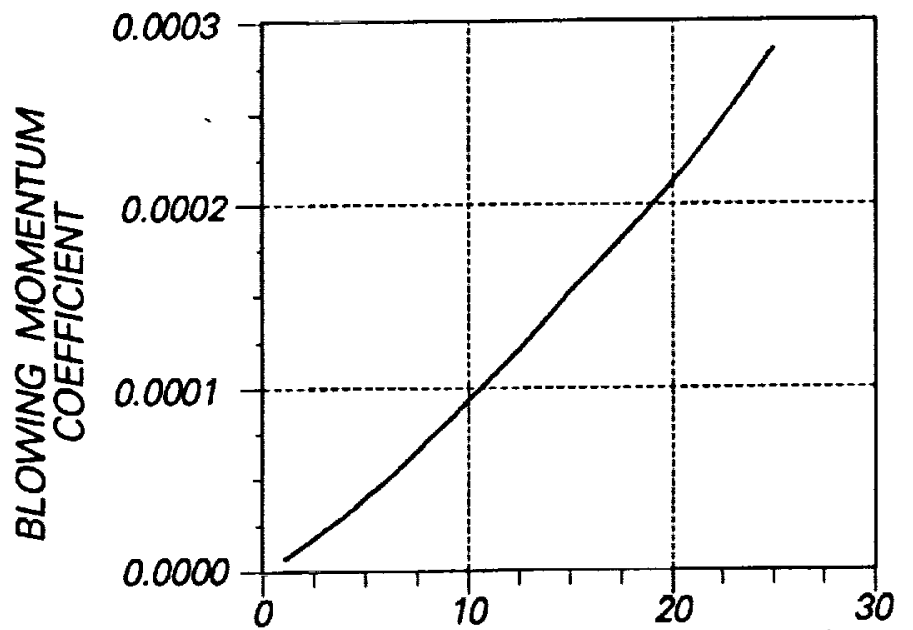

DIFFERENTIAL PRESSURE (psid)
Figure 43. Blowing Momentum Coefficient of Smoke at $q_{\infty}=18.28$ psf with $S=400 \mathrm{ft}^{2}$
Figure 43 shows how $C_{\mu}$ is predicted to vary with plenum chamber differential pressure for $18.28 \mathrm{psf}$ and $S=400 \mathrm{ft}^{2}$. The blowing momentum coefficient of the smoke entering the flow field is two orders of magnitude less than that which was shown to alter the vortical flow over strakes. The effect on forebody vortices is more difficult to predict and has not been adequately addressed in the open literature. More study is essential to determine minimum blowing momentum coefficients that significantly reposition forebody vortices.

\section{CONCLUSIONS AND RECOMMENDATIONS}

\section{Conclusions}

A prototype intermittent flow visualization system for use in studying the flow field around an aircraft in flight has been constructed and tested through the ground test phase. It releases discrete pulses of smoke consisting of particles of terephthalic acid. The pulses of smoke have sharp leading edges which remain coherent if the flow is not extremely turbulent. the are especially well-organized when entrained in a leading vortex core. This characteristic coherence allows tracking of the fluid elements using video-imaging technology. Once the time-dependent trajectory of a fluid element is known, its velocity and acceleration can be found by numerical differentiation.

A direct-acting three-way solenoid valve is used to pulse the smoke. Early in the development cycle a single solenoid valve with a spring return was used but it proved unreliable during ground firings. Residue from the chemical reaction built up in the valve body, often preventing the spring from shifting the spool that opened and closed the valve ports. After a double solenoid valve was installed, the valve functioned perfectly during all remaining tests in this environment.

Two methods of pulsing are effective. One method uses the valve to repeatedly divert the flow of smoke between two ports. Either or both ports may be used for visualization purposes. The other method requires the valve to repeatedly block the flow of smoke for short periods of time. This momentary blockage causes the pressure in the system to rise resulting in increased exit velocities of the smoke puffs.

A mathematical simulation of the system was developed and experimentally validated. This model is used to predict the pressure buildup in the system, as well as the exit velocity of the smoke, and a blowing momentum coefficient for the injected smoke. This coefficient allows comparison of the nondimensionalized momentum of the smoke as it enters the flow field with blowing momentum coefficients known to alter the vortical flow patterns. The largest blowing momentum coefficient expected to occur using the 
ISFVS is approximately two orders of magnitude less than that which has been shown to significantly affect the vortex lift created over leading edge strakes. However, considerably smaller blowing momentum coefficients apparently will change forebody vortex patterns. The minimum blowing momentum coefficients that will reposition forebody vortices has not been determined, but the largest coefficient expected to occur on the F-18 HARV when an ISFVS like the prototype is installed is still an order of magnitude less than the smallest blowing momentum coefficient that has been shown to alter forebody vortices significantly. But, considerably more research needs to be done to pin down this critical parameter for forebody vortices.

The model can be used to predict the effects of altering system parameters, such as the system volume and valve pulse rate, without actually modifying the system hardware. This versatility allows users to determine which method of pulsing the smoke suits their specific purposes.

The model is not without limitation, however. Since only a small number of cartridges have been fired and since the burn rates apparently varied considerably between cartridges, the maximum mass flow rate from a given cartridge remains uncertain. Also, the existing prototype only allows firing one cartridge at a time; hence, the effect of using multiple cartridges (to increase the density of the smoke) for each firing has not been experimentally verified.

\section{Recommendations}

The following recommendations, listed in approximate order of importance, are made for further testing of the system and the prediction model:

1) Test the system in a steady freestream with a velocity comparable to that of an aircraft in flight to determine its effect on the sharpness of the smoke puffs.

2) Test the system in a wind tunnel with a delta wing model at high angle of attack to ensure the increased exit velocity caused by blocking the flow of smoke repeatedly does not cause the smoke to miss the vortex core.

3) Fire several cartridges without cleaning the valve or other hardware to determine if multiple firings can be conducted in flight.

4) Conduct several tests with identical system parameters to obtain more data on how the burn rate varies from cartridge to cartridge.

5) Test different exit duct configurations to determine the effect of tubing length, diameter, and bend radii on the quality of the smoke puffs used for video imaging.

6) Determine allowable blowing momentum coefficients for using the visualization medium in different flow conditions.

7) Conduct tests to determine if valve preheating reduce residue buildup.

8) Compare data obtained in flight with predictions from computational fluid dynamics codes and with wind tunnel results. 


\section{REFERENCES}

1 Scott, W. B., "NASA Adds to Understanding of High Angle of Attack Regime," Aviation Week and Space Technology, May 22,1989, pp. 36-42.

2 Richwine, D. M., Curry, R.E., and Tracy, G.V., "A Smoke Generator System for Aerodynamic Flight Research," NASA, Edwards, CA, TM 4137, 1989.

3 United States Statutory Invention Registration No. H233, March 3, 1987.

4 Weast, R. C., CRC Handbook of Chemistry and Physics, 63rd ed., CRC Press Inc., Boca Raton, FL, 1982.

5 Windholtz, M., ed. The Merck Index: An Encyclopedia of Chemicals and Drugs, 9th ed., Merck and Co., Inc., Rahway, NJ, 1976, p. 1180.

6 "ExpertVision User's Manual," Motion Analysis Corporation, Santa Rosa, CA, 1989.

7 Morris, S.L., "A Video-Based Experimental Investigation of Wing Rock," Ph.D. Dissertation, Texas A\&M University, College Station, Texas, August 1989, pp. 119132.

8 Nelson, R.C., "Flow Visualization of High Angle of Attack Vortex Wake Structures," AIAA Paper 85-0102, Jan. 1985.

9 Rae, W.H., Jr., and Pope, Alan, Low-Speed Wind Tunnel Testing, 1st ed., John Wiley \& Sons, New York, 1984, pp. 129-140.

10 Merzkirch, W., Flow Visualization, 1st ed., Academic Press, New York, 1974, pp. 13-17.

11 Crowder, J.P., "Flow Visualization Techniques Applied to Full Scale Vehicles," Flow Visualization IV Proceedings of the Fourth International Symposium on Flow Visualization, 1st ed., Hemisphere Publishing Corporation, New York, 1987, pp. 21-22.

12 Fennell, L.J., "Vortex Breakdown - Some Observations in Flight on the HP115 Aircraft," NASA Reports and Memoranda N3805, September 1978.

13 Maltby, R.L. and Keating, R.F.A., "Smoke Techniques for Use in Low Speed Wind Tunnels," AGARDograph No. 70, North Atlantic Treaty Organization, 1962.

14 Del Frate, J., High Alpha Technology Program Workshop, NASA Ames Research Center-Dryden Flight Research Facility, Edwards, California, November 1,1989.

21 "Potter \& Brumfield Input/Output Modules," Potter \& Brumfield, Princeton, IN, 1990.

22 Conkling, J. A., Chemlstry of Pyrotechnics Basic Principles and Theory, Marcel Dekker, Inc., New York, 1985.

23 Kennan, C. W., et al, General College Chemistry, 5th ed., Harper and Row, New York, 1976, p. 703. 
24 Black, W. Z. and Hartley, J. G., Thermodynamics, Harper and Row, New York, 1985.

25 Daubert, T.E. and Danner, R.P., Data Compilation Tables of Properties of Pure Compounds, American Institute of Chemical Engineers, New York, 1985.

26 "Red Hat Flow Book," Automatic Switch Company, Florham Park, NJ, 1989.

27 Benedict, R. P., Fundamentals of Pipe Flow, John Wiley and Sons, New York, 1969, p. 409

28 Bradley, R. G. and Wray, W. O., "A Conceptual Study of Leading-Edge-Vortex Enhancement by Blowing," AIAA Journal of Alrcraft, Vol. 11, No. 1, January 1974, pp. 33-38.

29 Skow, A. M., et al, "Control of Forebody Vortex Orientation to Enhance Departure Recovery of Fighter Aircraft," AIAA Journal of Aircraft, Vol. 19, No. 10, October 1982 , pp. 812-819.

30 "The Procedure Handbook of Arc Welding," 12th ed., The Lincoln Electric Company, Cleveland, $\mathrm{OH}, 1973$, pp. 16.1-1 -- 16.1-29.

31 "User Manual for ATLAB," 2nd ed., Data Translation, Inc., Marlboro, MA, 1988. 


\section{APPENDIX A \\ FORTRAN SOURCE CODE FOR MODELING SYSTEM \\ PRESSURE AND EXIT VELOCITY}

A computer prediction code (written in FORTRAN for IBM-class personal computers) that models the ISFVS is included on the attached diskette. Conservation of energy and conservation of mass equations are integrated using a fourth order RungaKutta routine to determine the increase in the system pressure and visualization medium exit velocity. Simplifying assumptions made in this model are listed below.

1) The mixture inside the system behaves like an ideal gas with a gas constant of $0.139 \mathrm{~kJ} / \mathrm{kg}^{\circ} \mathrm{K}$.

2) Thermodynamic properties are not a function of the location inside the system (there is complete mixing).

3) The flow through the system inlets and exit is uniform.

4) The kinetic and potential energies of the mixture are negligible in comparison to the enthalpy.

5) No shaft work is down by the system.

6) The rate of energy entering the system by heat transfer is constant and can be found from the initial conditions.

7) The enthalpy of the mixture can be found using the method given by Daubert and Danner 25 .

8) The pressure losses in the exit duct are negligible.

9) The valve's flow capacity is a linear function of time as it opens and closes.

10) The mass flow rate through the valve can be found using gas flow graphs.

11) The smoke cartridge burns at a constant rate.

The resulting simplified conservation of mass equation becomes:

$$
\frac{d m_{\text {sys }}}{d t}=\sum_{\text {inlet }} \dot{m}-\sum_{\text {exit }} \dot{m}
$$

This expression says that the time rate of change of mass in the system equals the difference between the mass flow rate in and the mass flow rate out of the system. Similarly, the conservation of energy equation is:

$$
\frac{d E_{\text {sys }}}{d t}=\dot{Q}+\sum_{\text {inlet }} \dot{m} h-\sum_{\text {exit }} \dot{m} h
$$

The time rate of change of the energy in the system equals the rate at which energy is added to the system by heat transfer plus the energy added to the system entering minus the energy removed from the system by mass exiting.

Commented FORTRAN source code for the predictions described in this report are included in the floppy disk attached to the original of this report. The code is written in for and is compiled to run on DOS-based personal computers. In the interest of brevity the complete printout of the source code is not included. 


\section{APPENDIX B \\ DETAILED DRAWINGS OF INTERMITTENT SMOKE FLOW VISUALIZATION GENERATOR PROTOTYPE}

An exploded isometric of the prototype intermittent flow visualization system smoke generator is shown in Fig. 44. Detailed drawings of all parts of the smoke generator are shown in Figs. 45 through 54, followed by a parts list in Table 15. The circled numbers on the three-views correspond to the numbers on the parts list. All dimensions are in inches. Welding symbols follow the convention of the American Welding Society. GTAW indicates gas tungsten arc weld, also known as TIG weld.

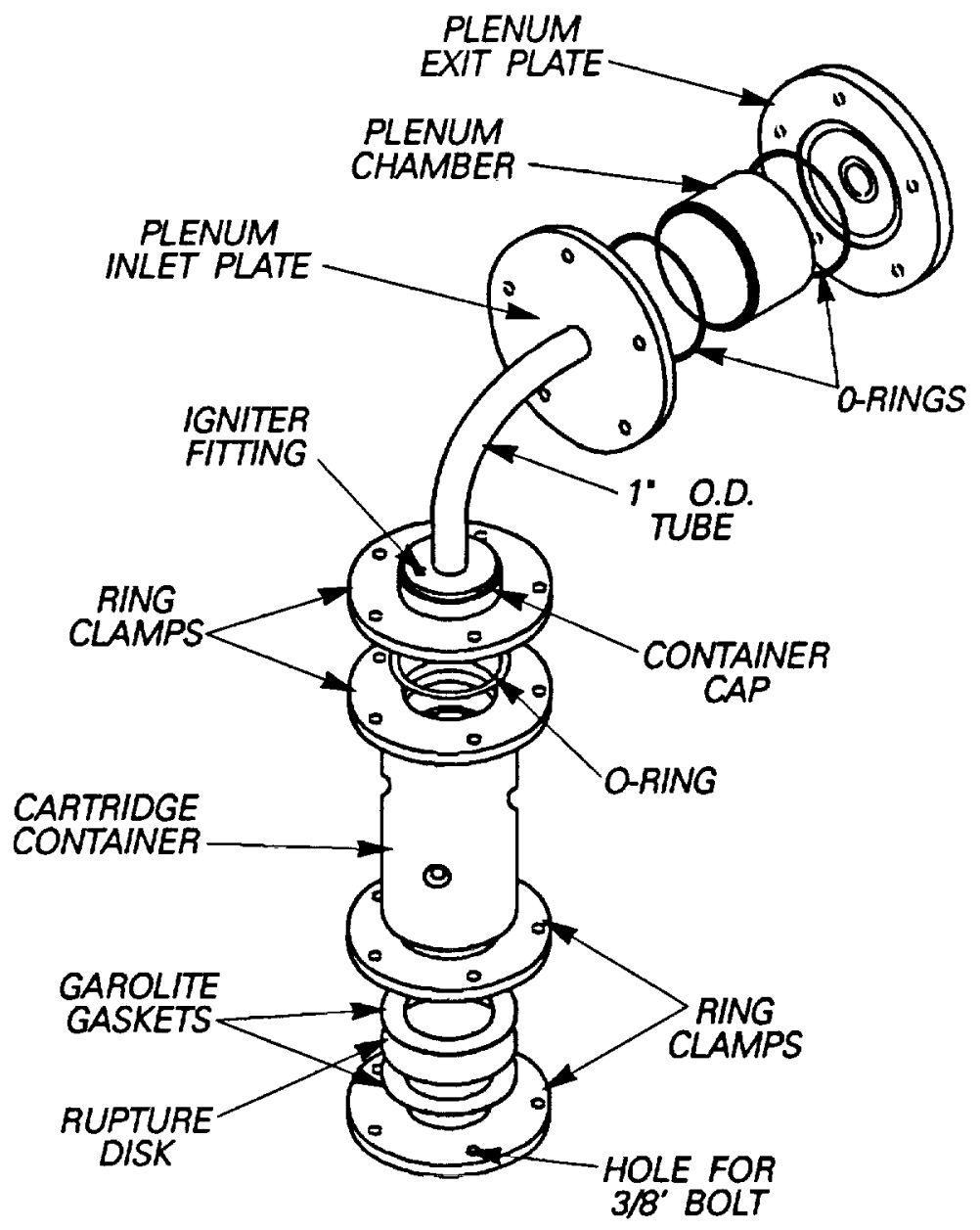

Figure 44. Exploded Isometric View of the Prototype ISFVS

Also included at the end of this appendix are specifications for the two types of valves used for the prototype ISFVS. 

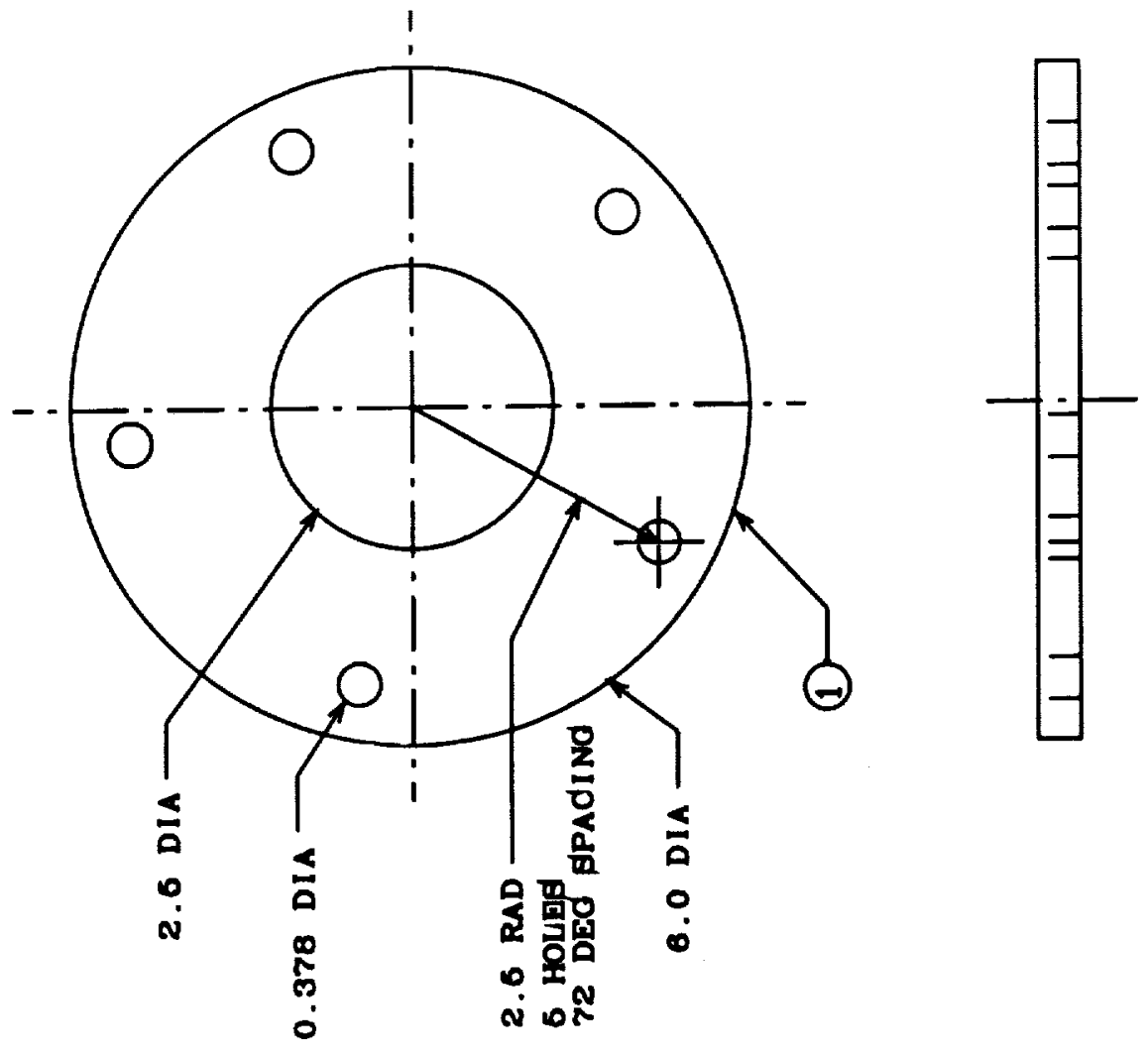

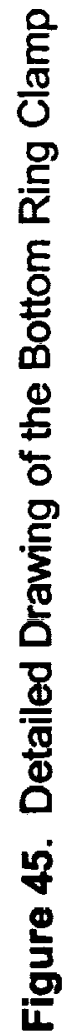

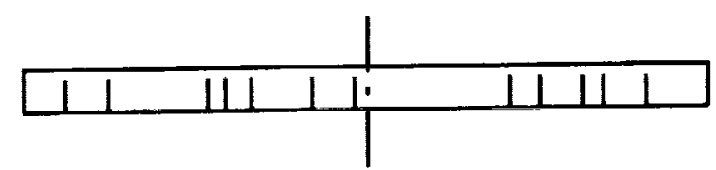



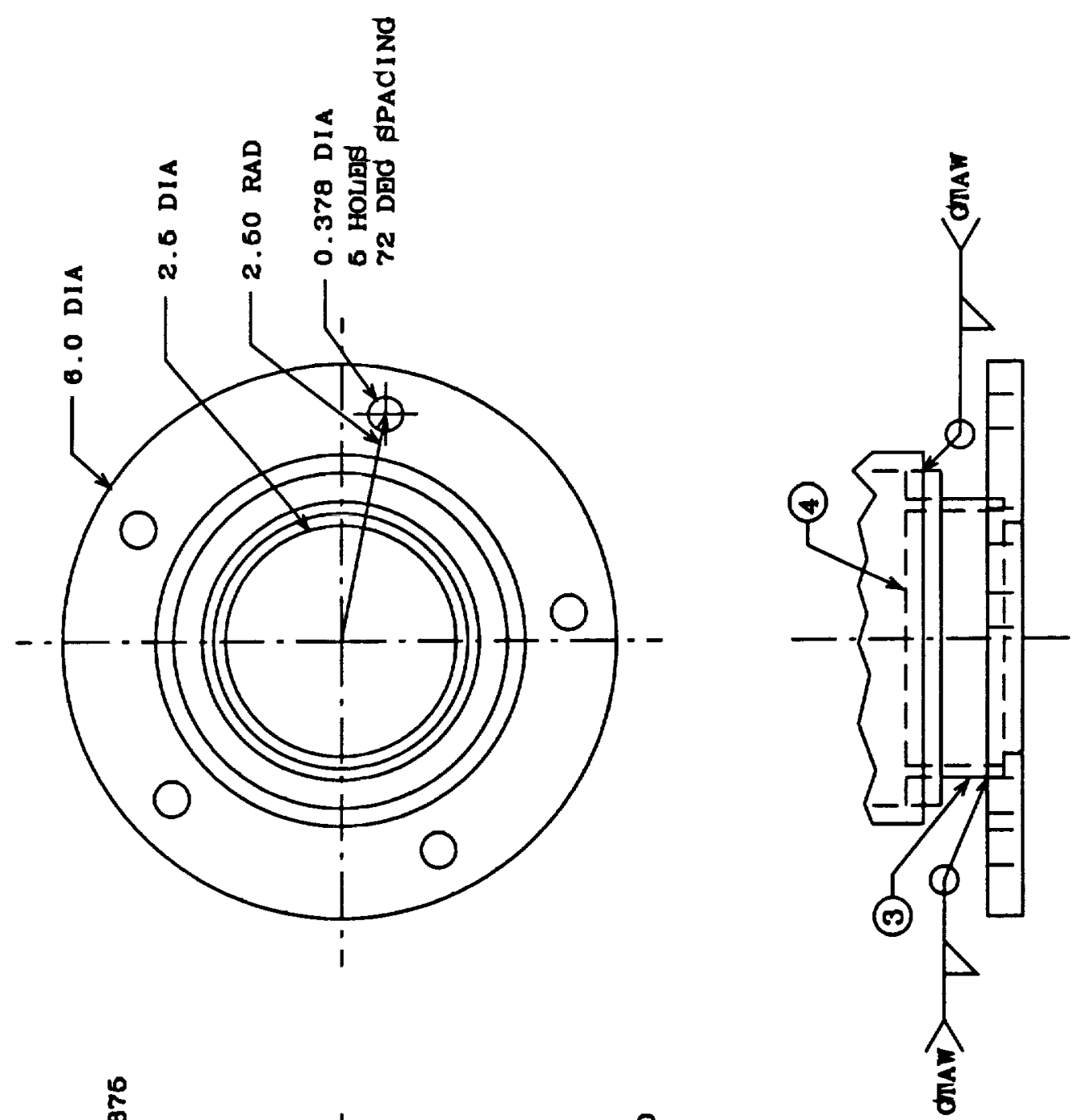

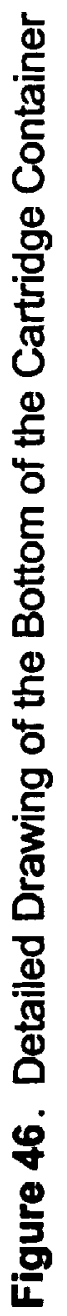

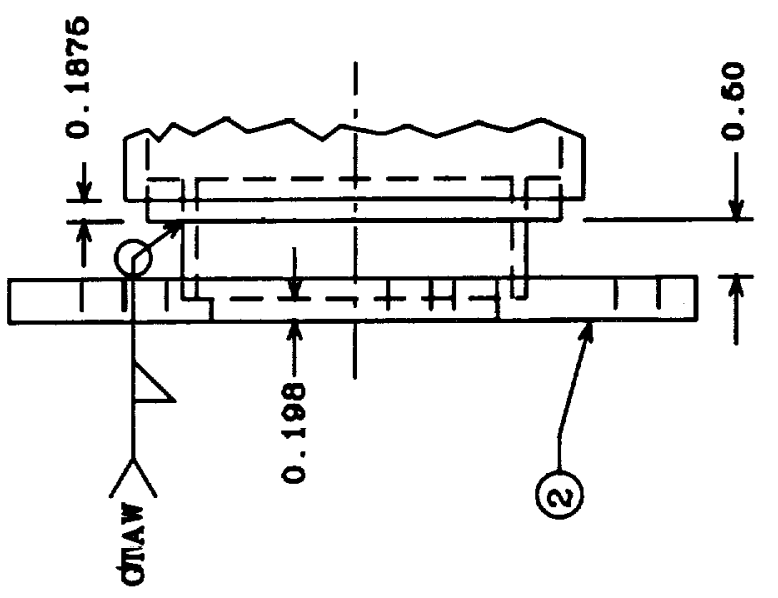




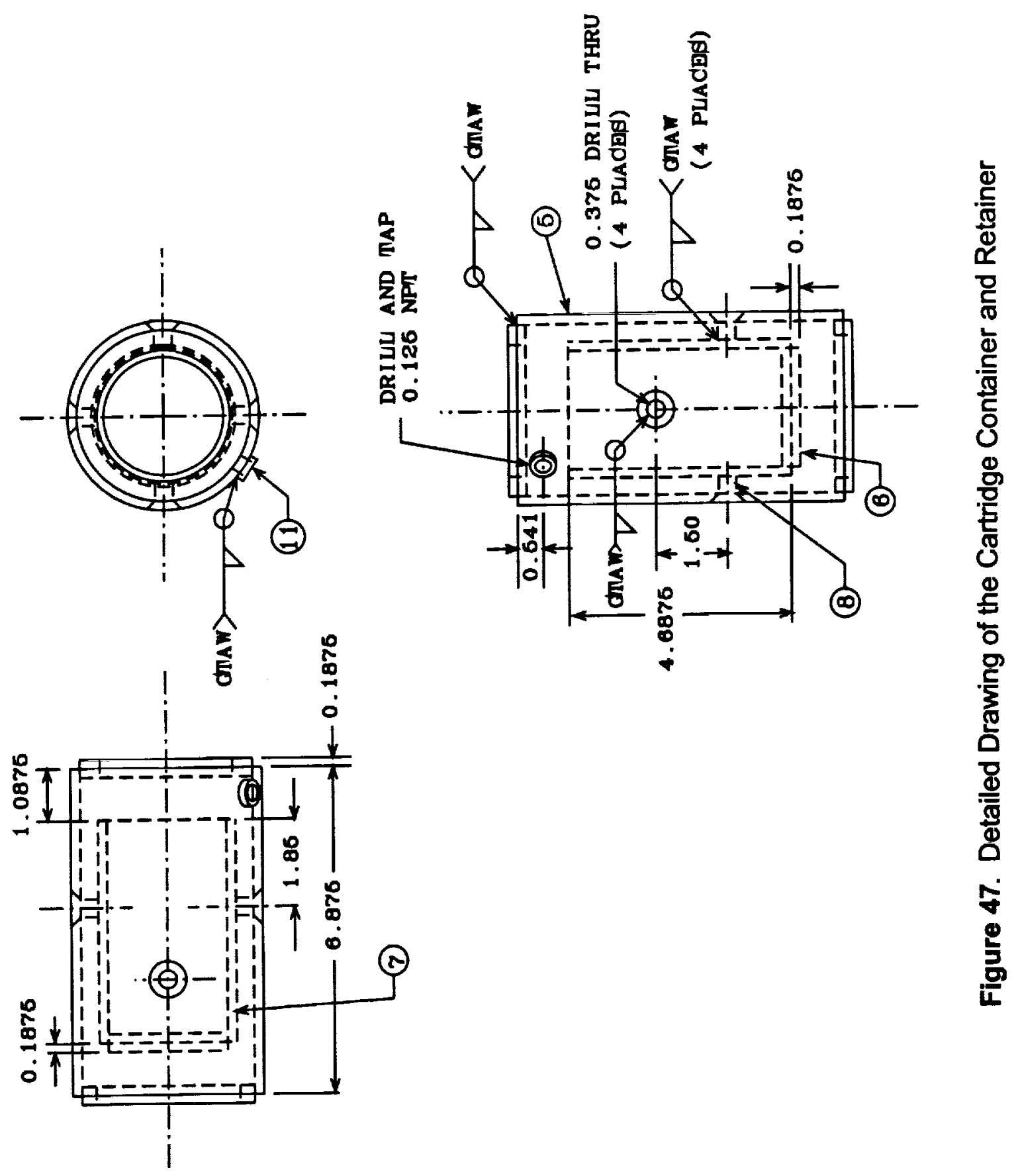



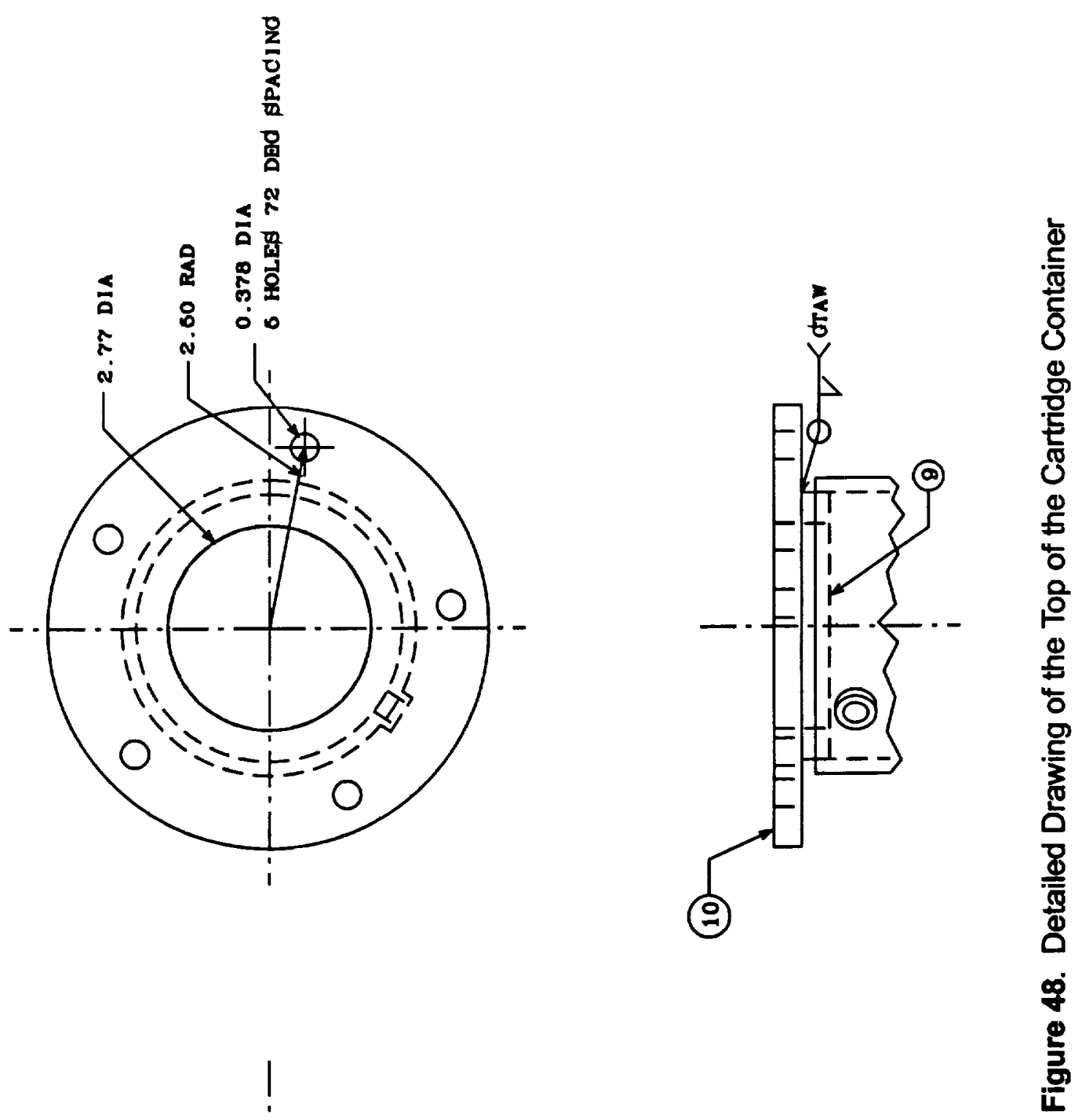

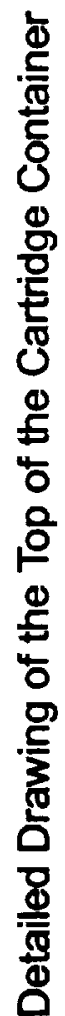

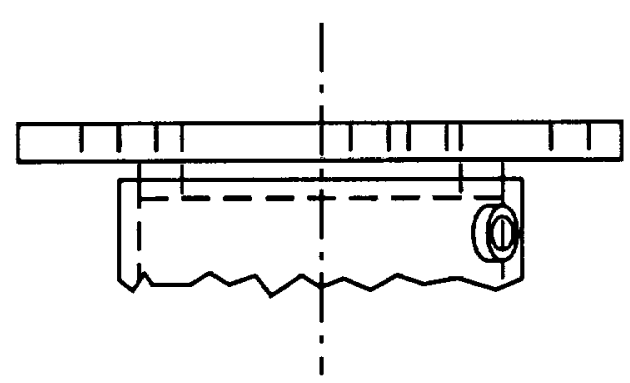




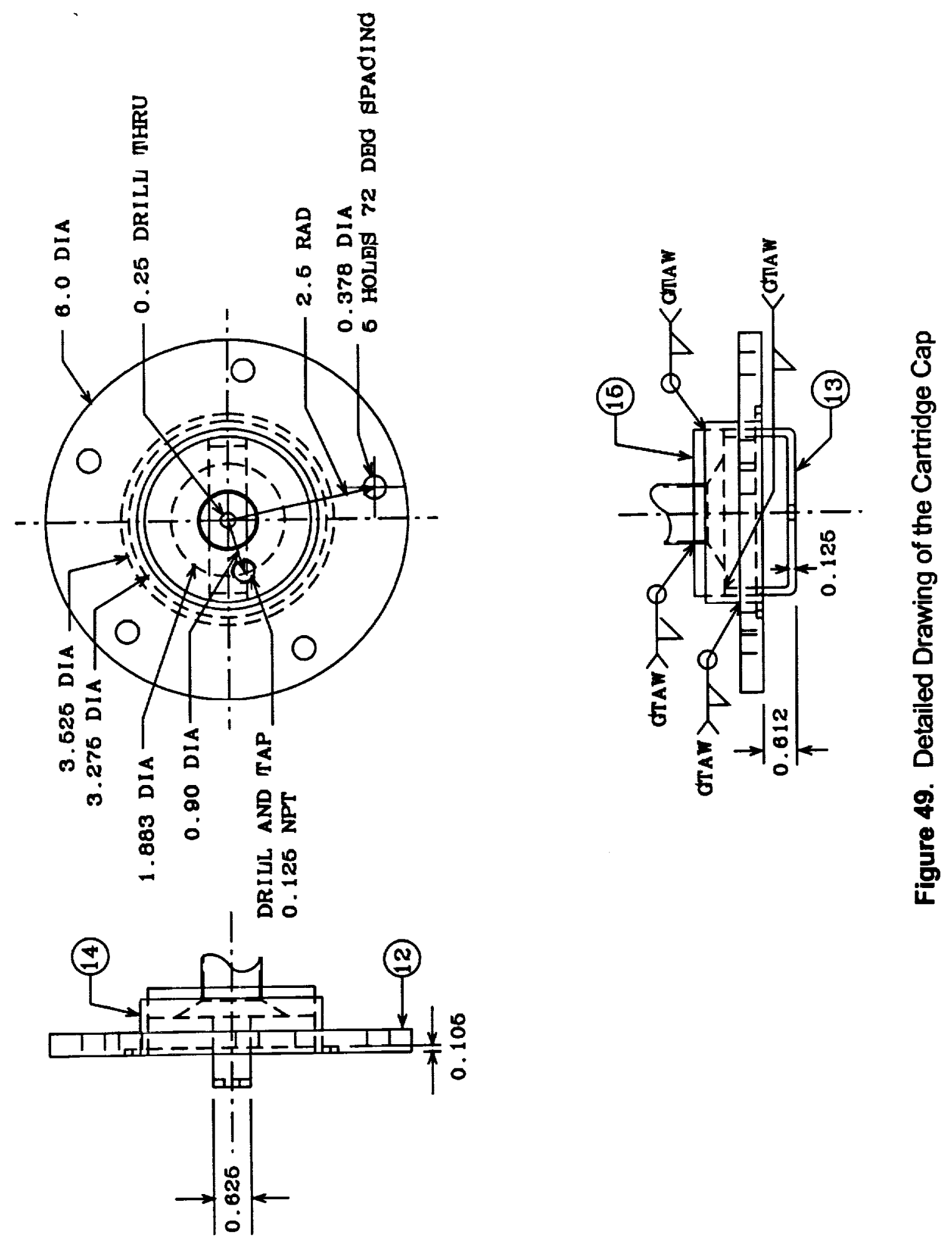



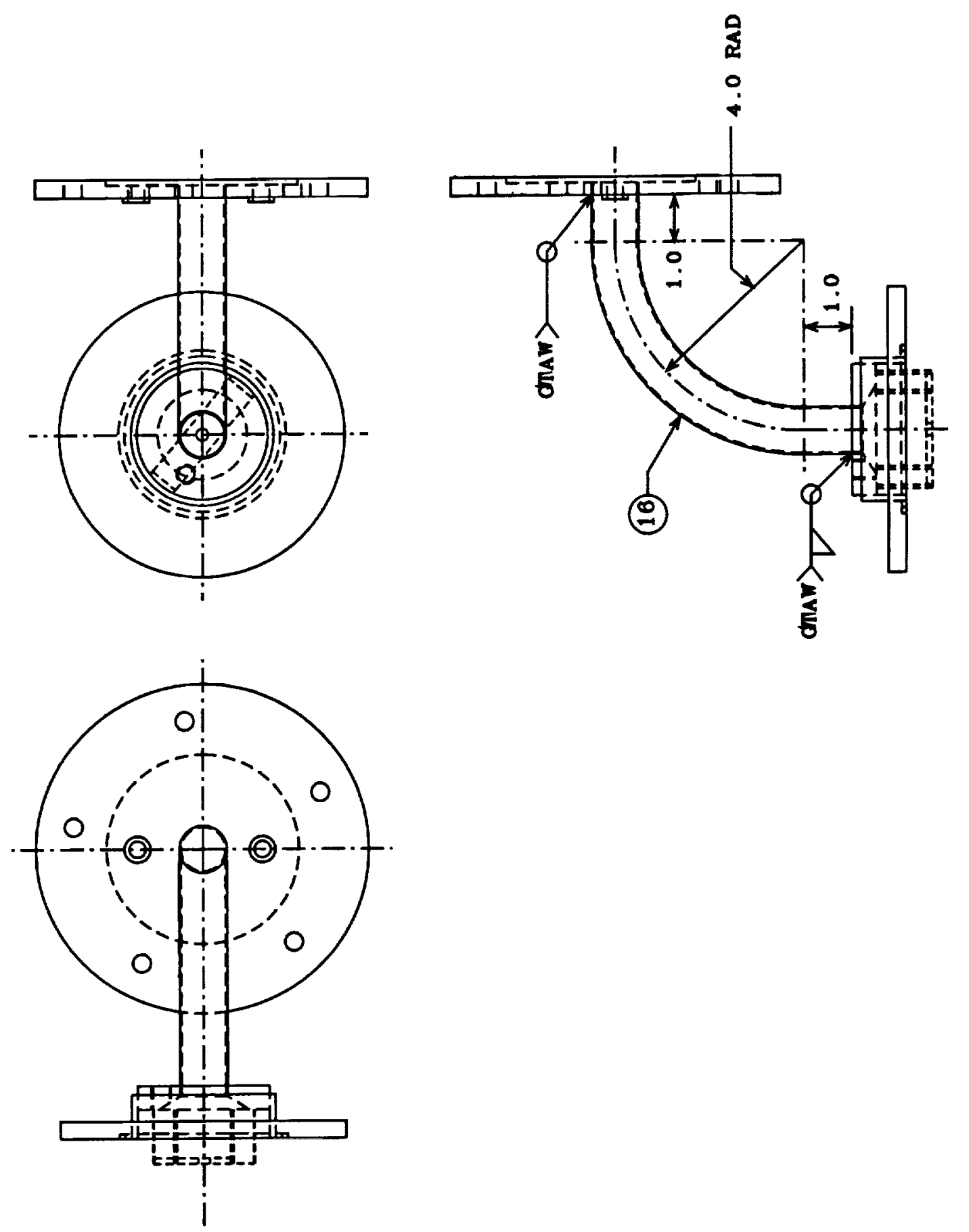

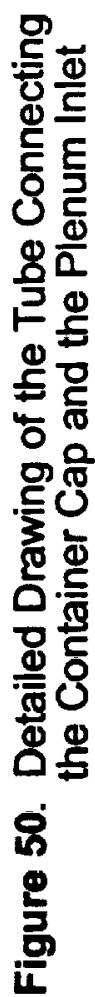




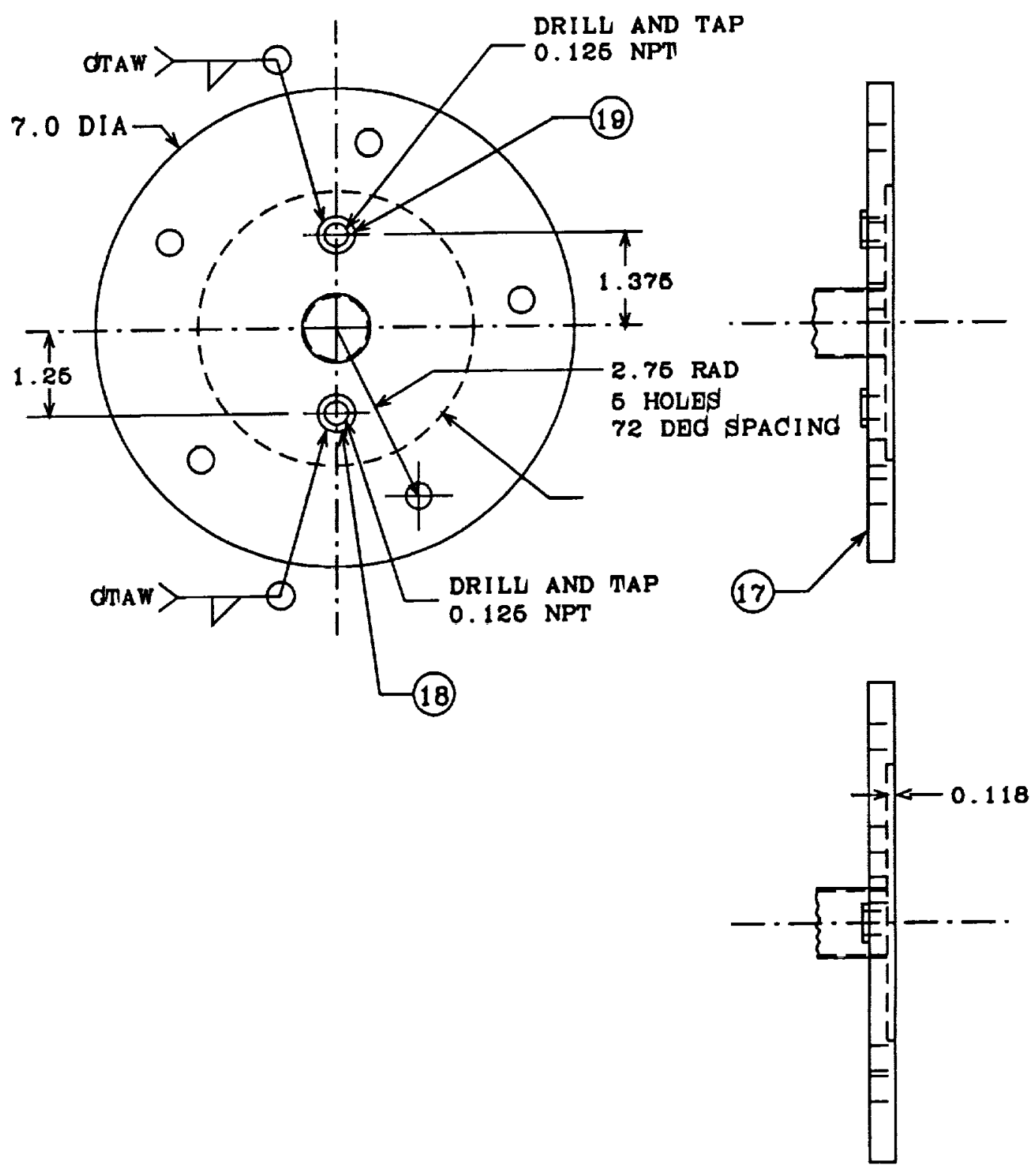

Figure 51. Detailed Drawing of the Plenum Chamber Inlet Plate 

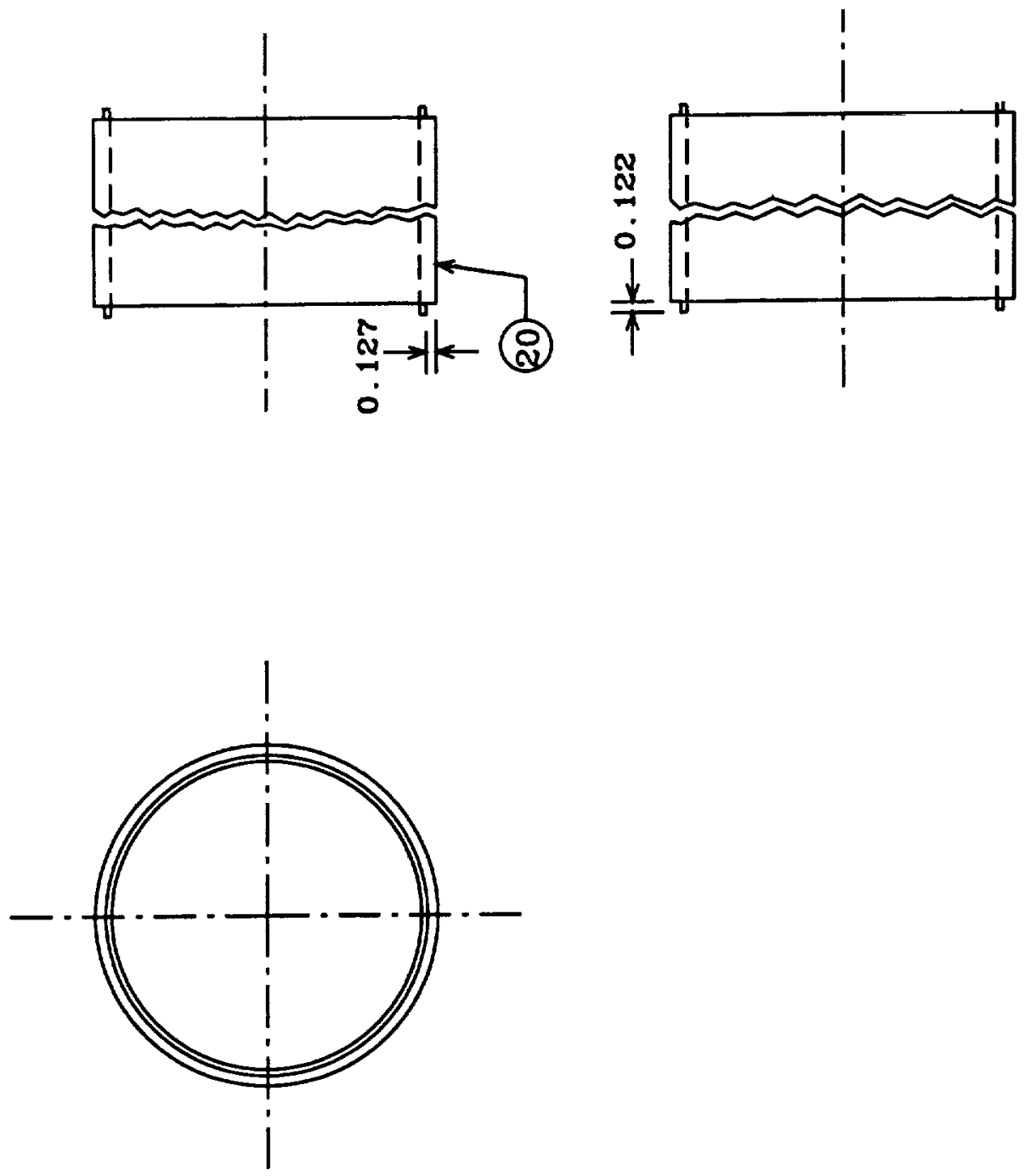

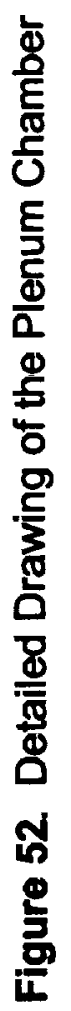




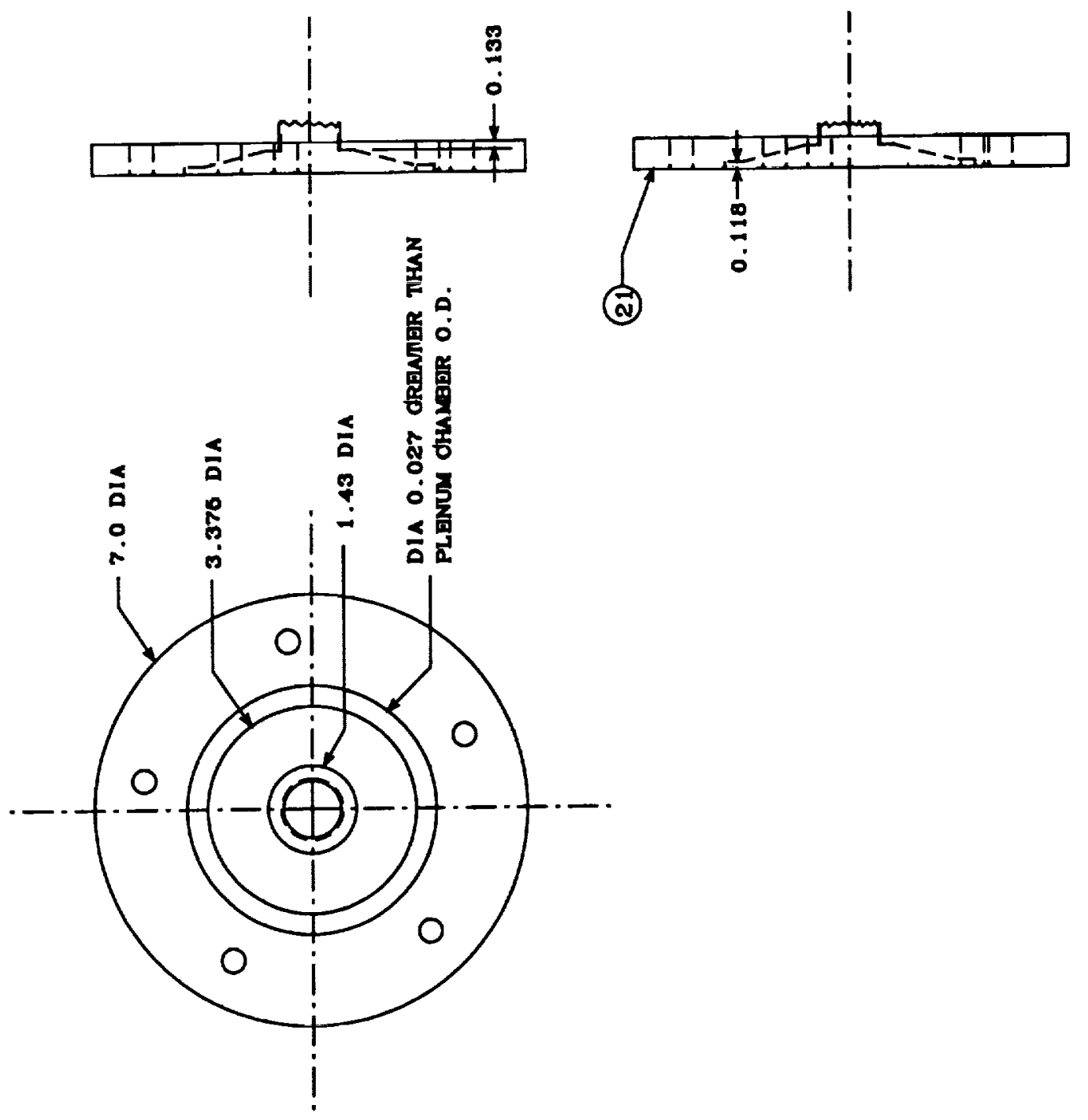

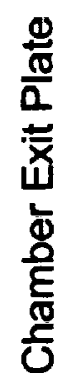

톡

믐

号

ธ。

高

in 

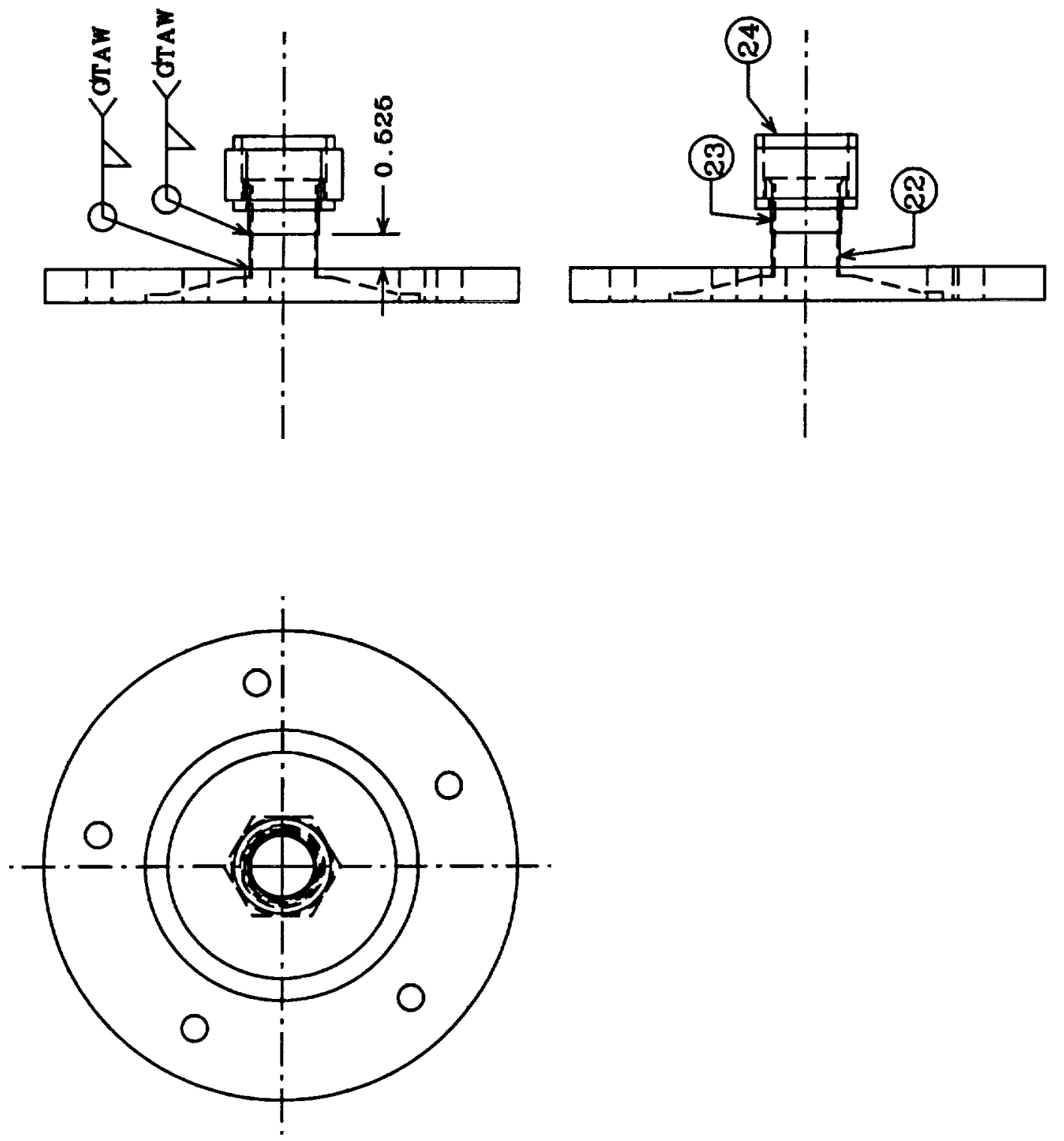

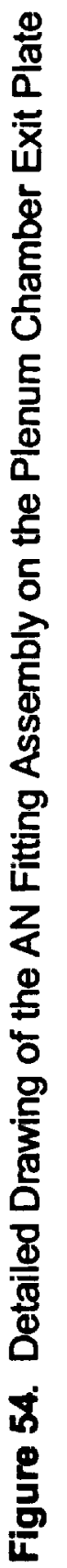


This page intentionally left blank. 
Table15. Smoke generator parts list

\begin{tabular}{cl} 
Part No. & Part \\
1 & Clamp Ring \\
2 & Clamp Ring \\
3 & Tubing \\
4 & Flange \\
5 & Cartridge Container \\
6 & Cartridge Retainer Bottom \\
7 & Cartridge Retainer \\
8 & Retainer Stand-offs (4) \\
9 & Flange \\
10 & Clamp Ring \\
11 & Thermocouple Fitting \\
12 & Clamp Ring \\
13 & Cartridge Stand-off \\
14 & Tubing \\
15 & Cap \\
16 & Duct \\
17 & Plenum Chamber Inlet \\
18 & Thermocouple Fitting \\
19 & Pressure Transducer Fitting \\
20 & Plenum Chamber \\
21 & Plenum Chamber Exit \\
22 & Duct \\
23 & Coupling Sleeve \\
24 & Coupling Nut \\
\hline
\end{tabular}

Material

3/8" AL 6061-T6 Plate

3/8" AL 6061-T6 Plate

3" X 1/8" AL 6061-T6 Tubing

3/8" AL 6061-T6 Plate

4" X 3/16" AL 6061-T6 Tubing

3/8" AL 6061-T6 Plate

$27 / 8$ " $\times 3 / 16$ " AL Tubing

3/8" Diameter AL 6061-T6 Rod

3/8" AL 6061-T6 Plate

3/8" AL 6061-T6 Plate

1/2 of AN 910-1D Fitting

3/8" AL 6061-T6 Plate

AL 6061-T6

3" X 1/8" AL 6061-T6 Tubing

1/2" AL 6061-T6 Plate

1" X 0.035" AL 6061-T6 Tubing

3/8" AL 6061-T6 Plate

1/2 of AN 910-1D Fitting

1/2 of AN 910-1D Fitting

4" X 3/16" AL 6061-T6 Tubing

1/2" AL 6061-T6 Plate

1" X 0.035" AL 6061-T6 Tubing

AN 819-16D Fitting

AN 818-16D Fitting 
Manufacturer:

Catalog:

Configuration:

Flow Capacity:

Port Size:

Operating Pressure Range:

Seals:

Response time:

Solenoids:

Single Solenoid:

Double Solenoid:

\section{Valve Specifications}

ISI Fluid Power, Fraser, MI

Detroit Line Air Valves for Industry

3-way

5.7

$3 / 4$ inch NPTF

28 " mercury vacuum to $125 \mathrm{psig}$

Viton (temperature range $400 \mathrm{~F}$ to $350 \mathrm{o}$ )

$12 \mathrm{msec}$

115 V $60 \mathrm{~Hz}$ AC Power

4.2 amps inrush, 0.6 amps holding

1 direct acting solenoid with spring return

part number 131-S-75

2 direct acting solenoids

part number 132-S-75 
Public reporting burden for this collection of Information is autimated 10 average 4 hour per responee, inctuding the time for roviewing instructions, searching existing data sources,

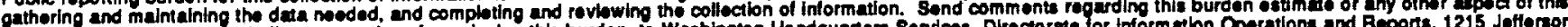

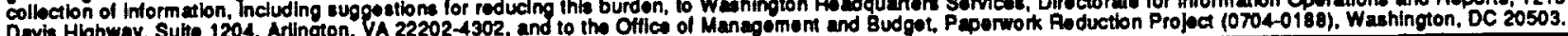

\begin{tabular}{|l|l|l|}
\hline 1. AGENCY USE ONLY (Loavo blank) & $\begin{array}{l}\text { 2. REPORT DATE } \\
\text { June } 1993\end{array}$ & $\begin{array}{l}\text { 3. AEPOAT TYPE AND D } \\
\text { Contractor Report }\end{array}$ \\
\hline
\end{tabular}

4. TITLE AND SUBTITLE

Preliminary Design of an Intermittent Smoke Flow Visualization System

\section{AUTHOR(\$)}

WU 533-02-35

NAG-2651

Donald T. Ward and James H. Myatt

7. PERFOAMING ORGANIZATION NAME(S) AND ADDAESS(ES)

Aerospace Engineering Division

Texas Engineering Experiment Station

Texas A \& M University

College Station, Texas 77843-3141

9. SPONSORING/MONITORING AGENCY NAME(S) AND ADDRESS(ES)

NASA Dryden Flight Research Facility

P.O. Box 273

Edwards, California 93523-0273

8. PERFORMING ORGANIZATION

REPORT NUMBER

TEES AERO TR 91-1

10. SPONSORING/MONITORING AQENCY REPORT NUMBER

NASA CR-186027

H-1917

\section{SUPPLEMENTARY NOTES}

NASA Dryden Technical Monitor: John Del Frate

12.. DISTRIBUTION/AVAILABILITY STATEMENT

12b. DISTRIBUTION CODE

Unclassified - Unlimited

Subject Category 05

\section{ABSTRACT (Maximum 200 words)}

A prototype intermittent flow visualization system has been designed to study vortex flow field dynamics has been constructed and tested through its ground test phase. It produces discrete pulses of dense white smoke consisting of particles of terephthalic acid by the pulsing action of a fast-acting three-way valve. The trajectories of the smoke pulses can be tracked by a video imaging system without intruding in the flow around in flight. Two methods of pulsing the smoke were examined. The simplest and safest approach is to simply divert the smoke between the two outlet ports on the valve; this approach should be particularly effective if it were desired to inject smoke at two locations during the same test event. The second approach involves closing off one of the outlet ports to momentarily block the flow. The second approach requires careful control of valve dwell times to avoid excessive pressure buildup within the cartridge container and does also increase the velocity of the smoke injected into the flow. The flow of the smoke has been blocked for periods ranging from 30 to 80 milliseconds, depending on the system volume and the length of time the valve is allowed to remain open between valve closings.

14. SUBJECT TERMS

Flow visualization; Smoke generation, Smoke grenades, Smoke pulsation

15. NUMBER OF PAGES 67

16. PRICE CODE A04

\begin{tabular}{|c|c|c|c|}
\hline $\begin{array}{l}\text { 17. SECURITY CLASSIFICATION } \\
\text { OF REPORT } \\
\text { Unclassified }\end{array}$ & $\begin{array}{l}\text { 18. SECURITY CLASSIFICATION } \\
\text { OF THIS PAGE } \\
\text { Unclassified }\end{array}$ & $\begin{array}{l}\text { 19. SECURITY CLASSIFICATION } \\
\text { OF ABSTRACT } \\
\text { Unclassified }\end{array}$ & $\begin{array}{l}\text { 20. LIMITATION OF ABSTRACT } \\
\text { Unlimited }\end{array}$ \\
\hline
\end{tabular}

